2. To: (Receiving Organization) Distribution

5. Proj./Prog./Dept./Div.:

SNF Project A.5/A.6

(Cask Transportation Facility Mod.)

8. Originator Remarks;

Determines the uncertainty associated with initial leak rates from a cask-MCO drop

For approval and release.

11. Receiver Remarks:

11A. Design Baseline Document? [] Yes [X] No
3. From: (Originating Organization)

K Bains Projects

6. Design Authority/Design Agent/Cog. Engr.

K. E. Ard / L. A. Rodgers / M. T. Hussain

4. Related EDT No.:

N/A

7. Purchase Order No.:

$\mathrm{N} / \mathrm{A}$

9. Equip./Component No::

$\mathrm{N} / \mathrm{A}$

10. System/Bldg./Facility:

$105 \mathrm{~K}$ West / East

12. Major Assm. Dwg. No.:

N/A

13. Permit/Permit Application No.:

N/A

14. Required Response Date:

ASAP

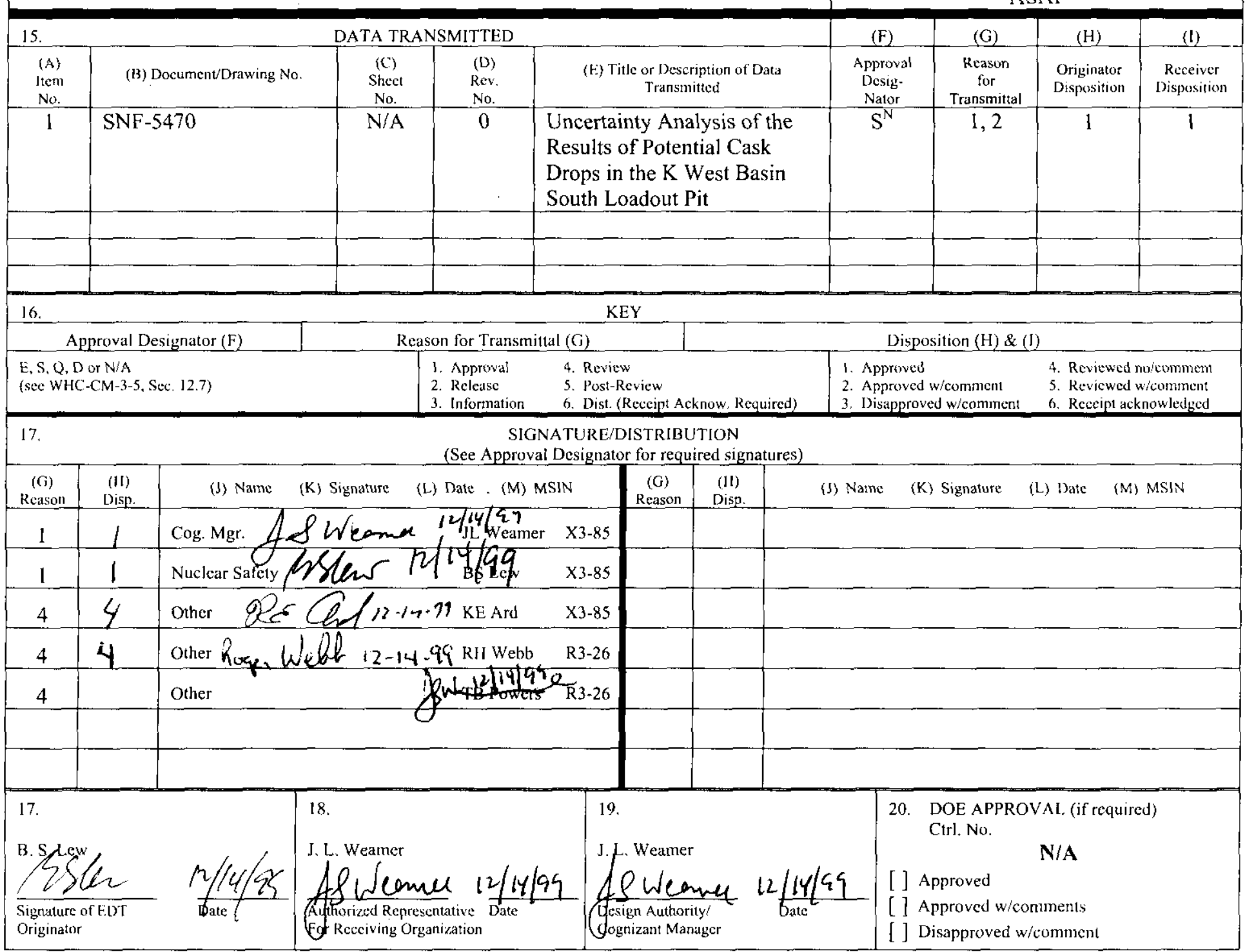




\section{DISTRIBUTION SHEET}

\begin{tabular}{|c|c|c|c|c|c|}
\hline \multirow{2}{*}{$\begin{array}{l}\text { To: } \\
\text { Distribution }\end{array}$} & \multirow{2}{*}{\multicolumn{3}{|c|}{$\begin{array}{l}\text { From: } \\
\text { K Basins Projects }\end{array}$}} & \multicolumn{2}{|l|}{ Page 1 of 1} \\
\hline & & & & \multicolumn{2}{|c|}{ Date: December 14, 1999} \\
\hline \multirow{2}{*}{\multicolumn{4}{|c|}{$\begin{array}{l}\text { Project Title/Work Order } \\
\text { Uncertainty Analysis of the Results of Potential Cask Drops in the K West Basin } \\
\text { South Loadout Pit - SNF-5470, Rev. } 0\end{array}$}} & EDT No. & 627341 \\
\hline & & & & ECN No. & $\mathrm{N} / \mathrm{A}$ \\
\hline Name & MSIN & $\begin{array}{l}\text { Text } \\
\text { With All } \\
\text { Attach. }\end{array}$ & $\begin{array}{l}\text { Text } \\
\text { Only }\end{array}$ & $\begin{array}{l}\text { Attach./ } \\
\text { Appendix } \\
\text { Only }\end{array}$ & $\begin{array}{c}\text { EDT/ECN } \\
\text { Only }\end{array}$ \\
\hline K. E. Ard & $\mathrm{X} 3-\overline{85}$ & $\overline{\mathrm{X}}$ & & & \\
\hline R. L. Boylston / R. L. Garrett & R3-26 & & & & $\mathrm{X}$ \\
\hline J. R. Ellis & $\mathrm{R} 3-26$ & $X$ & & & \\
\hline W. A. Frier & X3-76 & $X$ & & & \\
\hline K. D. Gibson / S. H. Peck / R. H. Webb & $\mathrm{R} 3-26$ & $X$ & & & \\
\hline B. S. Lew & $\mathrm{X} 3-85$ & $\mathrm{X}$ & & & \\
\hline P. L. Lingle * & $\mathrm{S} 2-51$ & $\mathrm{X}$ & & & \\
\hline T. B. Powers & $\mathrm{R} 3-26$ & $X$ & & & \\
\hline Central Files & B1-07 & $X$ & & & \\
\hline DOE/RL Reading Room (2 Copies) & $\mathrm{H} 2-53$ & $X$ & & & \\
\hline K Basin Project Files A.5/A.6 & $\mathrm{X} 3-85$ & $X$ & & & \\
\hline SNF Project Files & R3-11 & $\mathrm{X}$ & & & \\
\hline
\end{tabular}

\footnotetext{
* Advanced Copy
} 


\title{
Uncertainty Analysis of the Results of Potential Cask Drops in the K West Basin South Loadout Pit
}

\author{
B. S. Lew for \\ Fluor Daniel Hanford \\ Richland, WA 99352 \\ U.S. Department of Energy Contract DE-AC06-96RL13200 \\ EDT/ECN: $627341 \quad$ UC: $\quad 2000$ \\ Org Code: 2G400 Charge Code: 109477 \\ B\&R Code: EW7002010 Total Pages: 113
}

Key Words: SLOP, cask drop, leak rates, CTFM, A.5/A.6, uncertainty, and probability.

Abstract: If a cask and Multi-Canister Overpack (MCO) were to be dropped, the analysis established possible initial volumetric flow rates. The results of the uncertainty analysis demonstrated that even if a cask and $\mathrm{MCO}$ were to be dropped the possible initial volumetric flow rate through the opened floor/wall joint would be small. There is a $95 \%$ confidence (probability of 0.95 ) that the initial volumetric flow rate would be smaller than $411 \mathrm{gpm}$ and a $99 \%$ confidence (probability of 0.99 ) that it would be smaller than $916 \mathrm{gpm}$.

TRADEMARK DISCLAIMER. Reference herein to any specific commercial product, process, or service by trade name, trademark, manufacturer, or otherwise, does not necessarily constitute or imply its endorsement, recommendation, or favoring by the United States Government or any agency thereof or its contractors or subcontractors.

Printed in the United States of America. To obtain copies of this document, contact: Document Control Services, P.O. Box 950, Mailstop H6-08, Richland WA 99352, Phone (509) 372-2420; Fax (509) 376-4989.

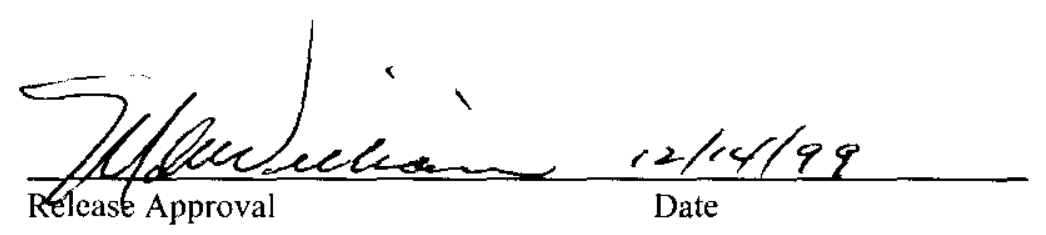

Approved for Public Release 
SNF-5470

Revision 0

\title{
UNCERTAINTY ANALYSIS OF THE RESULTS OF POTENTIAL CASK DROPS IN THE K-WEST BASIN SOUTH LOADOUT PIT
}

Prepared for

\author{
FLUOR DANIEL HANFORD \\ Contract No. 263, Release 54 \\ Line Item 60 \\ Report No. 9904561-001 \\ Revision 0
}

November 1999

Prepared by

\section{ARES CORPORATION \\ 636 Jadwin Avenue Suite B \\ Richland, Washington 99352}




\title{
UNCERTAINTY ANALYSIS OF THE RESULTS OF POTENTIAL CASK DROPS IN THE K-WEST BASIN SOUTH LOADOUT PIT
}

Prepared for

\author{
FLUOR DANIEL HANFORD \\ Contract No. 263, Release 54 \\ Line Item 60 \\ Report No. 9904561-001 \\ Revision 0
}

November 1999

Prepared by: Lewis D. Muhlestein, Ph.D. Approved by: Teurs D Mululestein Date: _ Nov.12,1999 


\section{EXECUTIVE SUMMARY}

The Spent Nuclear Fuel (SNF) Project will transfer SNF from the K-East and K-West Basins through the South Loadout Pit (SLOP) using the transfer bay crane and cask loading system. The initial basis for design, fabrication, installation, and operation of the cask handling and loading system was a worst case cask drop event that would not result in basin leakage. However, of the several cask drop analyses that have been performed, no attempt has been made to quantify the uncertainties or the risk associated with the various drop events.

This analysis: 1) determines the initial volumetric flow rate through the SLOP floor/wall joint gap assuming that a cask and Multi-Canister Overpack (MCO) have been dropped into the KWest Basin SLOP, and 2) determines the uncertainty associated with the initial volumetric flow rate. It is emphasized that the results are, therefore, conditioned on a cask and MCO being dropped into the SLOP. However, the likelihood of dropping a cask and MCO into the SLOP is very small and is preventable by following the $\mathrm{K}$-West Basin SLOP transfer bay crane lifting procedures.

If a cask and MCO were to be dropped, the analysis established possible initial volumetric flow rates by considering: 1) the uncertainty associated with the potential impact energy to the SLOP basin concrete floor, 2) the uncertainty associated with the transmission of the impact energy to yield the concrete floor and produce a resultant residual floor/wall gap, 3) the uncertainty in the resultant residual floor/wall gap separation, and 4) the likely flow rate through the floor/wall gap assuming flow through a porous medium.

The results of the uncertainty analysis demonstrated that even if a cask and MCO were to be dropped the possible initial volumetric flow rate through the opened floor/wall joint would be small. There is a 95 percent confidence (probability of 0.95 ) that the initial volumetric flow rate 
would be smaller than $411 \mathrm{gpm}$. The results of the uncertainty analysis are summarized in the following table.

\begin{tabular}{|l|l|l|}
\hline \multicolumn{1}{|c|}{ Summary Results } \\
\hline Mean & Probability & $\begin{array}{c}\text { Initial Volumetric Flow Rate Is Less } \\
\text { Than (gpm) }\end{array}$ \\
\hline 50th Percentile & & 51 \\
\hline 90th Percentile & 0.50 & 50 \\
\hline 95th Percentile & 0.90 & 268 \\
\hline 99th Percentile & 0.95 & 411 \\
\hline
\end{tabular}




\section{TABLE OF CONTENTS}

1.0 INTRODUCTION

2.0 DESCRIPTION OF K-WEST BASIN SLOP $\ldots \ldots \ldots \ldots \ldots \ldots \ldots \ldots \ldots \ldots$

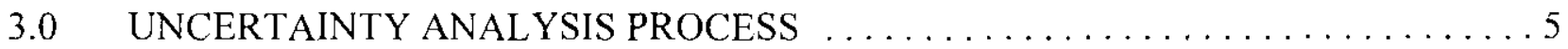

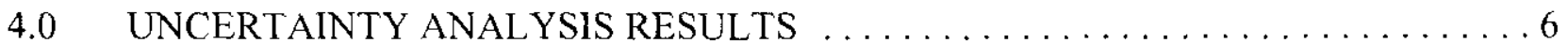

$4.1 \quad$ Impact Energy Distribution for Cask and MCO Drops $\ldots \ldots \ldots \ldots \ldots$

4.2 Wall-Floor Joint Deformation Predictions . . . . . . . . . . . . . . . 9

4.3 Impact Energy and Associated Floor/Wall Gap Crack Diameter . . . . . . . 10

$4.4 \quad$ Flow In A Porous Medium . . . . . . . . . . . . . . . . . . . . . . . . . . . 12

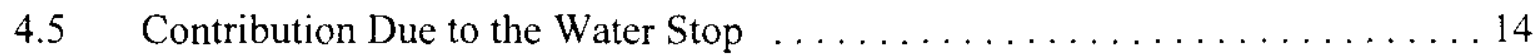

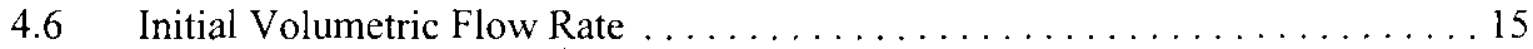

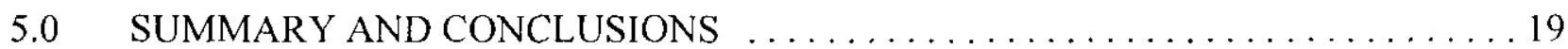

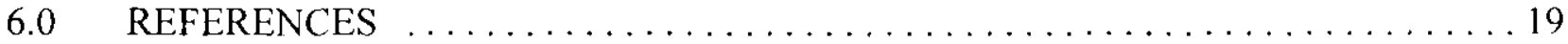

\section{APPENDICES}

Appendix A

Impact Energy Distribution for Cask/MCO Drops $\ldots \ldots \ldots \ldots \ldots \ldots \ldots \ldots$ A-1 Appendix B

K Basin SLOP Wall-Floor Joint deformation Predictions . . . . . . . . . . . . . B-1

Appendix C

Impact Energy and Associated Concrete Crack Diameter ............... C-1 Appendix D

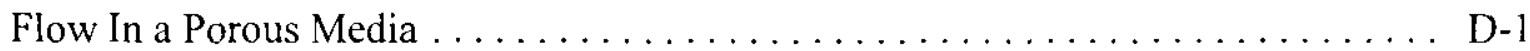

Appendix E

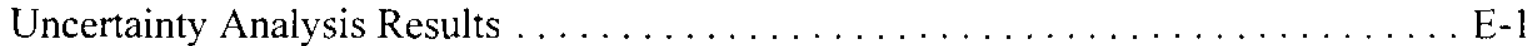

Appendix F

Validation of Analysis Models $\ldots \ldots \ldots \ldots \ldots \ldots \ldots \ldots \ldots \ldots \ldots \ldots \ldots \ldots \ldots$ 


\section{FIGURES}

Figure 1. Plan View of the K-West Basin South Loadout Pit

Showing the Immersion Pail Support Structure. .................... 3

Figure 2. Partial Elevation View of the K-West Basin South Loadout Pit

Showing the Immersion Pail Support Structure. . . . . . . . . . . . . . . . 4

Figure 3. Probability Distribution for the Impact Energy That Could be

Imparted to The SLOP Concrete Floor by a Dropped Cask. . . . . . . . . . . . 8

Figure 4. Uncertainty in the Residual Gap as a Function of the Impact Energy. . . . . . . . . 11

Figure 5. Cumulative Distribution Function of the Residual Floor/Wall Gap. . . . . . . . . 13

Figure 6. Probability Density Function for the Initial Volumetric Flow Rate. . . . . . . . . . 16

Figure 7. Cumulative Distribution Function for the Initial Volumetric Flow Rate. . . . . . . 17

\section{TABLES}

Table 1. Summary Results of Impact Energy That Could Be Imparted to SLOP Concrete Floor by a Dropped Cask . . . . . . . . . . . . . . . . 9

Table 2. Residual Floor/Wall Gap Predictions for the Cask Drop Cases and Drop Scenarios ................................... 10

Table 3. Volumetric Flow Rate for Flow Through a Porous Medium for Various Floor/Wall Gap Distances . . . . . . . . . . . . . . . . . . . . . 14

Table 4. Probability of Not Exceeding a Specified Initial Volumetric Flow Rate . . . . . . 18 


\section{ACRONYMS}

IPSS Immersion Pail Support Structure

MCO Multi-Canister Overpack

SLOP South Loadout Pit

SNF Spent Nuclear Fuel 


\subsection{INTRODUCTION}

The Spent Nuclear Fuel (SNF) Project will transfer SNF from the Hanford 105 K-East and 105 $\mathrm{K}$-West Basins to safe interim storage in the Canister Storage Building in the 200 Area. The fuel transfer from the K-East and K-West Basins will be conducted through the South Loadout Pit (SLOP) using a cask loading system. The empty Multi-Canister Overpack (MCO) and cask will be lifted from the transport trailer and placed into an immersion pail that is contained in the Immersion Pail Support Structure (IPSS) in the SLOP. The immersion pail is used to minimize contamination of the MCO and cask during the fuel loading operation. The IPSS is used to suspend and support the immersion pail containing the MCO and cask at the desired elevation in the SLOP.

The initial basis for the design, fabrication, installation, and operation of the cask handling and loading system in the SLOP was a worst case cask drop event that would not result in excessive basin leakage. Several analyses of various cask drop scenarios have been performed over the past several years. One of the latest analyses was that of an immersion pail support structure drop into the SLOP (Moore 1997). An independent review of this analysis (Wagenblast 1999) was completed which concluded that the analysis did not address all potential failure modes. The analysis results of Kanjilal (1999) also indicate that previous cask drop analyses were not conservative in their assumptions and loading conditions. The analysis of Kanjilal (1999) used more detailed boundary conditions in the finite element model and considered the loads at the joint between the concrete slab and the concrete wall in the SLOP. No attempt has been made in any of the analyses to quantify uncertainties associated with the various drop events.

The objectives of this analysis are to: 1) determine the initial volumetric flow rate through the SLOP floor/wall joint gap assuming that a cask and MCO have been dropped into the K-West Basin SLOP, and 2) determine the uncertainty associated with the initial volumetric flow rate. It is emphasized that the results are, therefore, conditioned on a cask and MCO being dropped into the SLOP, and the first line of defense is to prevent a cask and MCO from being dropped into the SLOP. The likelihood of dropping a cask and MCO into the SLOP is very small and is preventable by following the K-West Basin SLOP transfer bay crane lifting procedures.

The initial volumetric flow rate uncertainty analysis is a risk-based uncertainty analysis where best engineering judgement is used to represent each variable in the analysis. The uncertainty associated with each variable is represented by a probability distribution (probability density function). Uncertainty exists because of the normal randomness associated with the distribution of values that a variable may assume, and because of a lack of knowledge concerning a variable. Engineering judgement and technical information are used to develop the variable probability density functions, while the bounds of the probability density function are based on physical limitations. The uncertainty, described by probability distributions, is propagated through the 
analysis by Monte Carlo convolution techniques. The corresponding results are developed as a probability distribution and the risk is expressed in terms of the corresponding complementary cumulative distribution function ("risk curve"). The total risk is the area under the "risk curve".

An uncertainty analysis is well suited for evaluating the possible results of a cask drop in the KWest Basin SLOP. Only very conservative information or analyses exist relative to precisely how a dropped cask might impact the concrete floor, damage the concrete, and produce cracks through which the basin water may be released. If such analyses were to be completed they would be lengthy to perform and may provide results that would be difficult to interpret.

On the other hand, an uncertainty analysis can provide very good results in the form of a risk curve with the uncertainty in the variables represented by probability density functions. To be effective, the uncertainty distributions used for each variable must be represented by realistic and physical bounds, and the median value of the distribution must be generated using the best available engineering judgment. In this analysis some of the variable distributions were developed by using the results of more detailed models. By using realistic distributions to represent both the randomness of variables and the lack of information, the exact details become less important.

The following sections provide summaries of: 1) background information relative to the risk analysis process, 2) a description of the K-West Basin SLOP cask loading system, 3) the evaluation process, and 4) the results of the risk analysis process. Technical details and specific analyses required to support the information provided in the main sections of this document are provided in Appendices A, B, C, D, and E. Appendix A develops the distribution for the impact energy that may impact the SLOP concrete floor due to specific and random crane drop scenarios. Appendix B is the finite element analysis of concrete wall-joint deformation for various cask drop accidents. The correlation between the impact energy and the associated residual floor/wall gap distance is developed in Appendix $\mathrm{C}$ using the information of Appendix B. Appendix D discusses fluid flow in a porous media and presents the information necessary to determine the volumetric flow rate for flow through a porous medium. Appendix $E$ brings all of the information together and completes a Monte Carlo convolution process to evaluate the distribution for the volumetric flow rate given a cask load drop occurs. Appendix F contains a validation of the analysis models and a record of the review comments received relative to this document and the corresponding changes that were made based on the review comments. It is important to realize that a great deal of information and analyses are developed and presented in the appendices. Only summary information is presented in this main body of the report. 


\subsection{DESCRIPTION OF K-WEST BASIN SLOP}

A plan view of the K-West Basin SLOP is shown in Figure 1; a partial elevation view is shown in Figure 2. Figure 1 shows the eight vertical rails that are permanently attached to the concrete walls of the SLOP that are used to position the IPSS. The IPSS is shown in Figure 1 and partially shown in Figure 2 with its four leg structures. Each leg is supported by a leveling foot.

Rails Typ

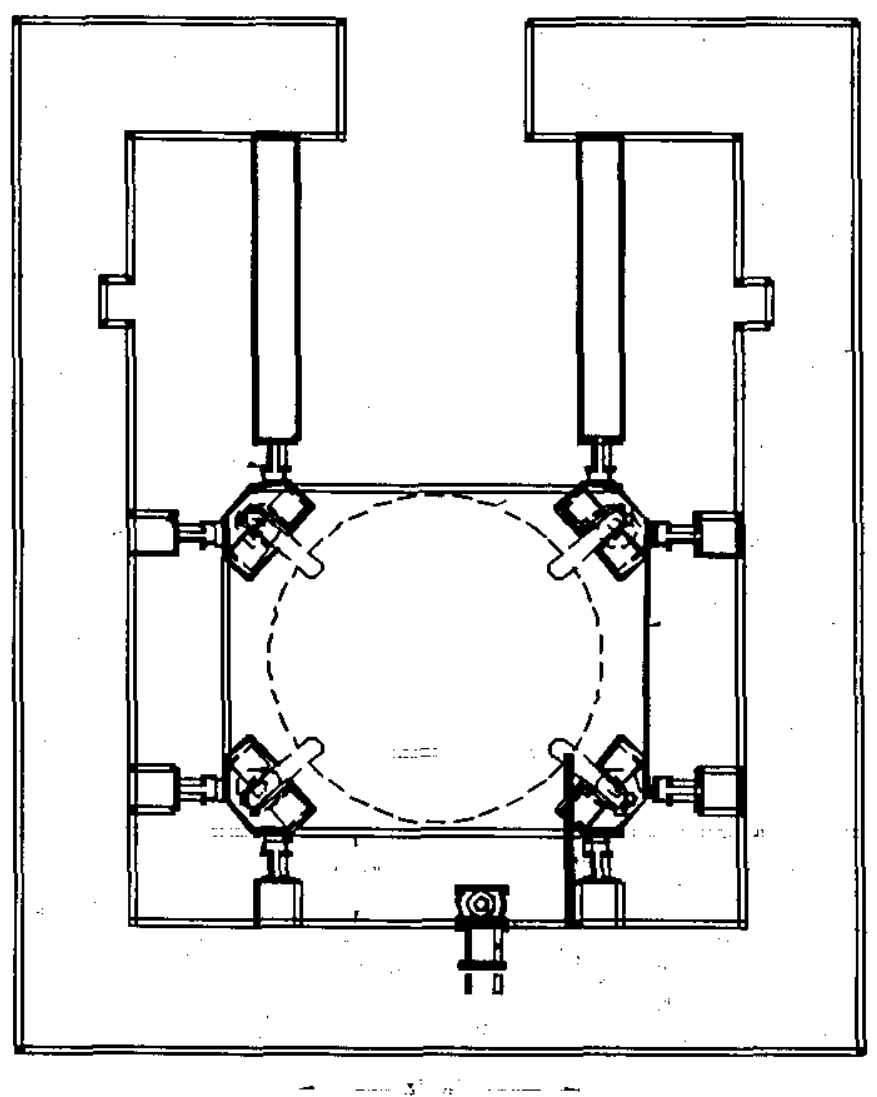

Immersion Pail

Support Structure

Concrete Filled

Sump

4" Gate Valve

\section{Figure 1. Plan View of the K-West Basin South Loadout Pit Showing the Immersion Pail Support Structure.}

Figure 2 also shows the water stop that was placed in the concrete to help form a water-tight seal between the concrete floor and the concrete wall. Also depicted in Figure 2 is the floor/wall gap between the concrete floor and the concrete wall that could be formed by a sufficient impact 


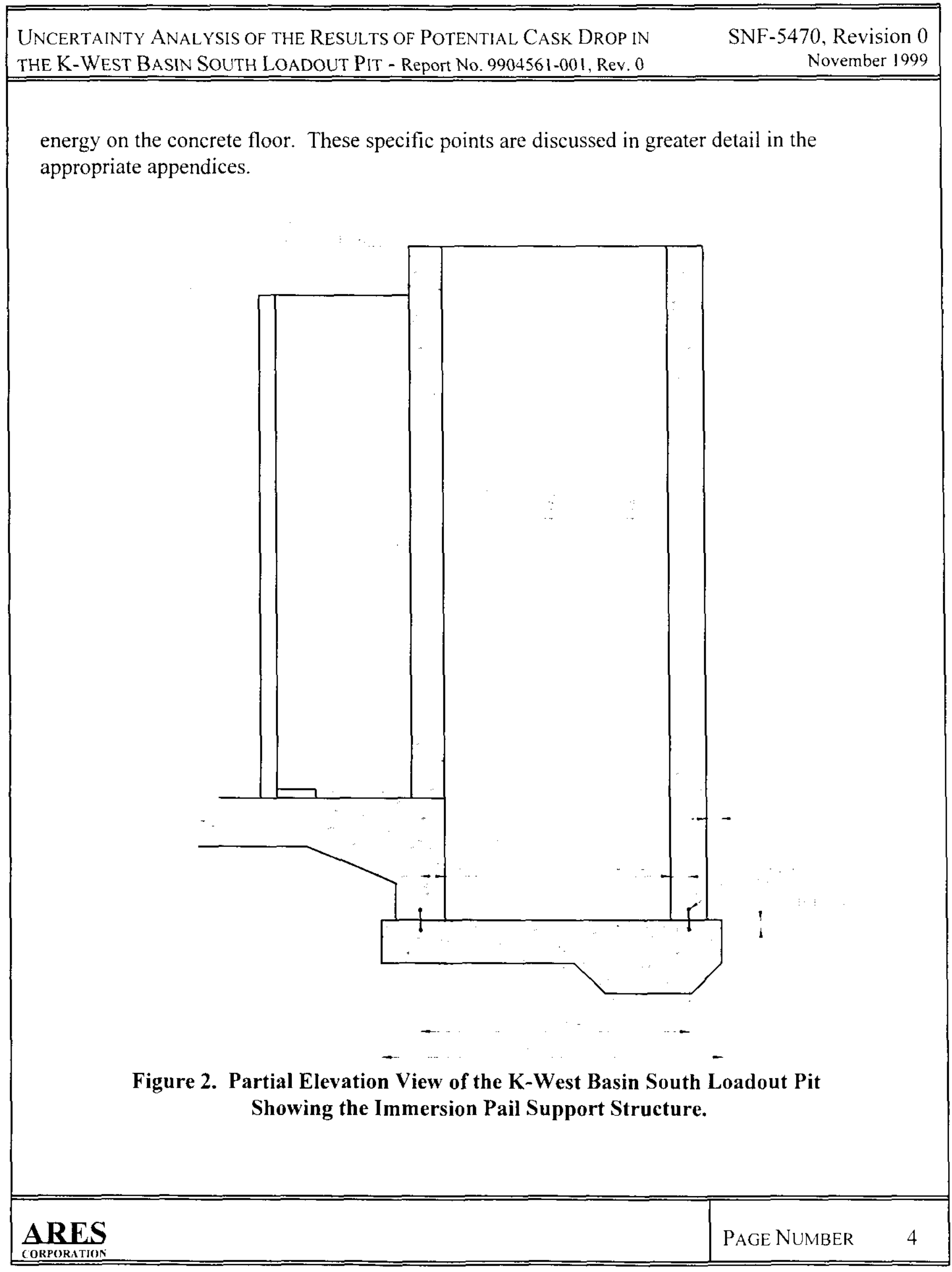




\subsection{UNCERTAINTY ANALYSIS PROCESS}

The uncertainty analysis considers a sequence of events which assumes that a cask and MCO have been dropped into the K-West Basin SLOP. The uncertainty analysis then considers the potential impact of the dropped cask and $\mathrm{MCO}$ on the concrete basin floor, damage of the floor/wall joint, and potential water flow through the damaged floor/wall joint. Several probability density function which represent specific information relative to the analysis are combined to provide the results that may be expected should a cask and $\mathrm{MCO}$ be dropped into the SLOP. Each of the probability density functions are representative of conditional probabilities. That is, probabilities that are conditional on a previous event occurring.

The conditional probabilities are determined based on the uncertainty associated with each of the variables involved in each individual calculation. The conditional probabilities are combined in a final model which is solved using Monte Carlo convolution techniques. In this analysis it was expeditious to partition the solution into various sections in order to examine each portion of the analysis to gain additional insight into the results.

The initiating event of the analysis is the assumption that a load has been dropped into the $\mathrm{K}$ West Basin SLOP using the transfer bay crane. As noted earlier, the likelihood of this event is very small.

Given the assumption of a load drop by the transfer bay crane, the next event is the distribution for the potential impact energy that the basin concrete floor may experience from a variety of load configurations which include several cask/MCO configurations, drop heights, and absorbing energies. This distribution is developed in Appendix A and represents the conditional probability of an impact energy given that a load drop occurs.

The dependence of the residual floor/wall gap on the impact energy was developed in Appendix $\mathrm{C}$ using the wall-floor joint deformation predictions from Appendix $\mathrm{B}$. The result was then used to develop a probability distribution for the residual gap in the floor/wall joint given an impact energy on the concrete.

Given a residual gap in the floor/wall joint, the next conditional probability distribution developed was the initial volumetric flow of water though the gap considering flow through a porous medium. The results are given in Appendix D.

The three conditional probabilities were convoluted using Monte Carlo convolution techniques to consider all of the uncertainties associated with the applicable variables. The four steps combined in the model are: 1) the probability of an impact energy given a load drop (Appendix A), 2) the residual floor/wall gap dependence on the impact energy (Appendix B and C), 3) the 
probability of the residual floor/wall gap given an impact energy (Appendix C), and 4) the potential volumetric flow rate through the floor/wall gap assuming flow through a porous medium (Appendix D and E).

\subsection{UNCERTAINTY ANALYSIS RESULTS}

The results of the uncertainty analysis are presented in the order discussed above in the process. The initiating event of the analysis is the assumption that a load has been dropped in the K-West Basin SLOP using the transfer bay crane.

\subsection{Impact Energy Distribution for Cask and MCO Drops}

The potential impact energy that could be experienced by the concrete at the bottom of the KWest Basin SLOP was determined given that a cask was dropped by the transfer bay crane. Potential cask/MCO drop configurations were identified in five vertical drop scenarios. The drop scenarios (see Appendix A, Figures A-1, A-2 and A-3) are:

(1) Dropping a cask and empty MCO from the nominal transfer bay crane height and impacting the immersion pail. The cask, empty $\mathrm{MCO}$ and immersion pail fall and impact the concrete at the bottom of the SLOP.

(2) Dropping a cask, empty $\mathrm{MCO}$, and immersion pail from the immersion pail locking position height. The cask, empty MCO and immersion pail impact the concrete.

(3) Dropping a cask, loaded MCO and immersion pail from a few feet above the bottom of the SLOP. The cask, loaded MCO and immersion pail fall and impact the concrete at the bottom of the SLOP.

(4) Dropping a cask, loaded MCO and immersion pail from the immersion pail locking position height. The cask, loaded MCO and immersion pail fall and impact the concrete at the bottom of the SLOP.

Dropping a cask and loaded MCO from the nominal crane lifting height and impacting the immersion pail. The cask, loaded $\mathrm{MCO}$, and immersion pail fall and impact the concrete at the bottom of the SLOP.

Gravitational, buoyant and drag forces were considered for each of the vertical drop scenarios. The equation of motion was solved in closed form (see Appendix A) to determine the velocity of the falling mass. If the object fell first in air the velocity equation was solved twice; once to 
evaluate the velocity at the end of the fall in air, and the second to evaluate the velocity at the end of the fall in water. The final velocity of the first solution was used as the initial velocity for the second solution.

The buoyant force of the immersion pail was determined including the foam attached to the lower two-thirds of the outside of the immersion pail (see Appendix A). Also included was the five inches of foam which is being designed to be attached to the bottom of the immersion pail as an impact limiter.

The velocity of the object just before impact was determined from which the kinetic energy at impact was determined. The energy lost due to the impact of the cask and MCO with the bottom of the immersion pail and the subsequent shearing of the pins that secure the immersion pail to the immersion pail support structure was determined. The energy to shear the immersion pail locking pins was subtracted from the kinetic energy before impact to determine the kinetic energy after impact. The kinetic energy after impact was used to determine the initial velocity for the second part of the drop scenario.

The velocity of the object just before hitting the bottom of the immersion pail support structure was evaluated and used to determine the kinetic energy just before impact. The energy absorbed by the impact limiter at the bottom of the immersion pail was determined as well as the energy absorbed by the loaded immersion pail striking the bottom of the immersion pail support structure. The kinetic energy of the system was reduced by the energy absorbed by the impact limiting foam and the energy absorbed by yielding the bottom of the immersion pail support structure. This final kinetic energy was assumed to be the energy that impacted the concrete at the floor of the SLOP basin.

Each of the variables involved in the impact energy calculation were represented by appropriate probability density functions. It was assumed that a drop from any height was equally possible. The weight of the total cask was determined by randomly varying between two states; either a loaded MCO or an empty MCO. If a loaded MCO was chosen, then the mass considered was a cask and loaded MCO for drop heights above the immersion pail. For drop heights below the immersion pail the mass considered was a cask, loaded $\mathrm{MCO}$ and immersion pail. If an empty MCO was chosen then the mass considered was a cask and empty $\mathrm{MCO}$ for drop heights above the immersion pail and a cask, empty MCO, and immersion pail for drop heights below the immersion pail. Out of the four lifts for each $\mathrm{MCO}$, half of the lifts will be with a loaded MCO and the other half will be with an empty MCO. This result was verified with a nearly equal 50:50 split between the two states.

The results of this analysis was a distribution for the impact energy that could be imparted to the SLOP concrete floor if a cask were to be dropped. The results are presented in Figure 3. 
Because of all of the parameters that were varied in the analysis the scatter in the data was more than is usual for an analysis of this type. However, the data were fitted to a probability density function. The probability density function that best fit the data was a Weibull probability density function. A Lognormal probability density function did not have an extensive enough tail in the distribution to adequately represent the larger impact energies that were observed in the data. The fitted probability density function is used to represent the impact energy from a dropped cask in the remainder of the uncertainty analysis.

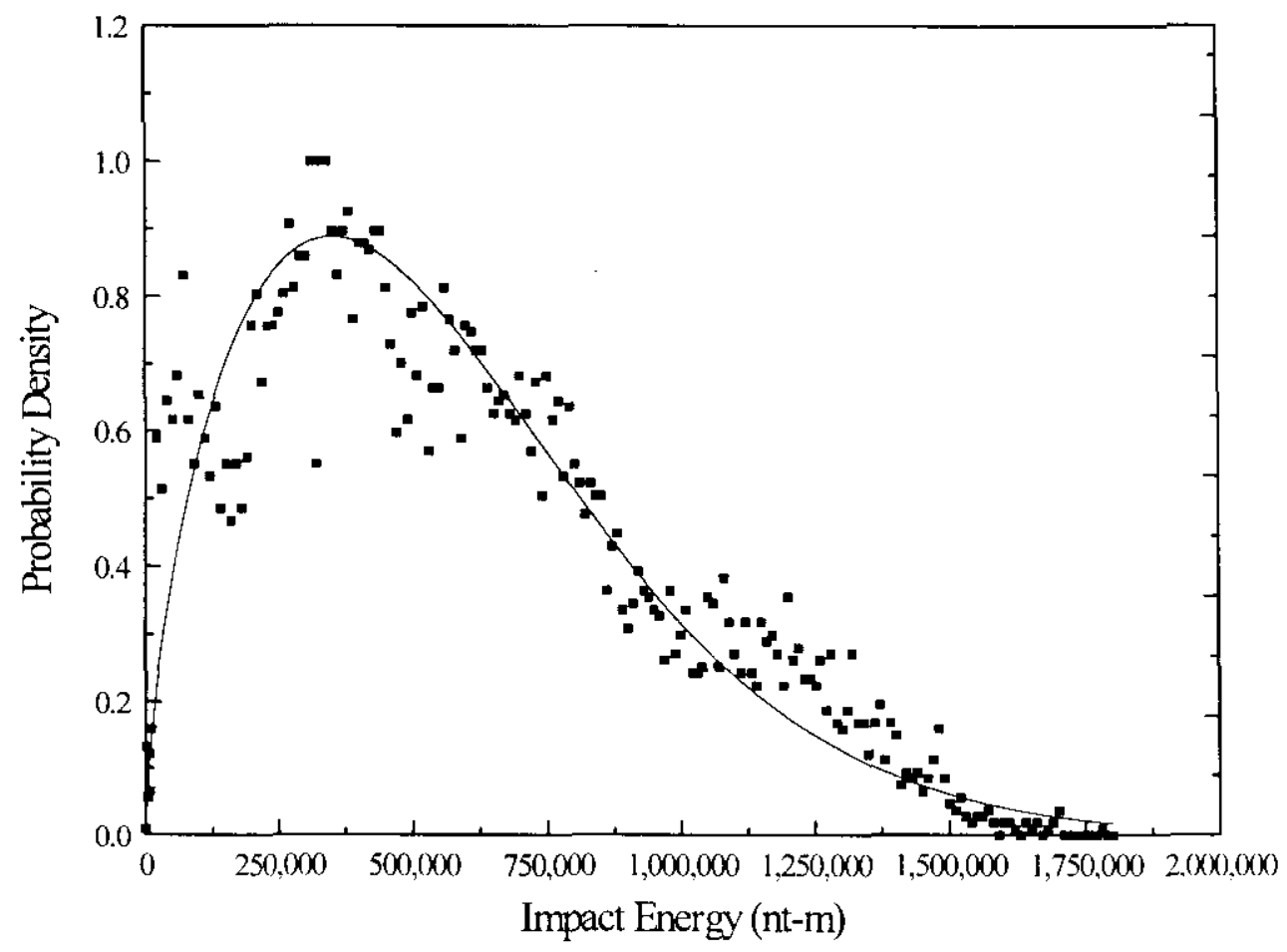

Figure 3. Probability Distribution for the Impact Energy That Could be Imparted to The SLOP Concrete Floor by a Dropped Cask.

A summary of the results presented in Figure 3 is provided in Table 1 which gives the impact energy that could be imparted to the SLOP concrete floor should a cask be dropped by the SLOP transfer bay crane. The parameters that defined the Weibull probability density function that was the best fit to the data (solid line in Figure 3) are also listed in Table 1. 
Table 1. Summary Results of Impact Energy That Could Be Imparted to SLOP Concrete Floor by a Dropped Cask

\begin{tabular}{|c|c|c|}
\hline \multirow[b]{2}{*}{ Percentile } & \multicolumn{2}{|c|}{ Impact Energy Imparted to SLOP Concrete Floor } \\
\hline & $(n t-m)$ & $(\mathbf{f t}-\mathbf{l b})$ \\
\hline 50 th & 394,400 & 290,700 \\
\hline 90 th & $1,059,690$ & 781,070 \\
\hline 95 th & $1,224,050$ & 902,220 \\
\hline 95 th & $1,448,600$ & $1,067,740$ \\
\hline \multicolumn{3}{|c|}{ Weibull Parameters for Fitted Distribution } \\
\hline Location Parameter & Scale Parameter, $n$ & Shape Parameter, $\beta$ \\
\hline 0 & $639,756 \mathrm{nt}-\mathrm{m}$ & 1.61 \\
\hline
\end{tabular}

\subsection{Wall-Floor Joint Deformation Predictions}

The potential deformation in the SLOP Basin concrete joint between the basin floor and the basin wall was evaluated and included as Appendix B. Based on the analysis in Appendix A, a range in potential concrete impact energies was provided to M\&D Professional Services Inc. from which several finite element model analyses were completed. The input for the finite element analysis in Appendix B was an impact energy and the corresponding impact velocity. The intent was to provide a large distribution of impact energies for which a large distribution of floor/wall residual gap dimensions could be developed. The impact energy corresponds to the weights of the objects given in Table 1-1 of Appendix B for the various vertical drops scenarios. The cask drop cases and corresponding drop scenarios evaluated are listed in Table 2.

Two sets of analyses were completed; one using the best available values for the input parameters and one using conservative values for the input parameters. Based on prior experience in evaluating cask drops on the SLOP concrete floor, the primary leak path following a cask drop is the construction joint between the SLOP concrete floor and the concrete walls. Therefore, a finite element model of the lower portion of the SLOP was developed which focused on the wall/floor joint. The finite element model was used to perform a transient dynamic analyses for each of the 12 cask drop cases listed in Appendix B, Table 1-1. From the dynamic analysis results, residual gap predictions for the floor/wall joint were obtained for each 
drop case. Immediately following the time of impact, the maximum gap opening occurs, followed by some gap closure due to foundation soil rebound. The remaining "residual gap" is the item of interest for predicting leakage flow.

For the lower impact energy drops, inelastic stretching of the joint rebar was limited to minor yielding in the middle of the walls of the SLOP. For the other impact energy drops, significant yielding of the joint rebar occurred in all but the east wall (the wall furthest away from the immersion pail support structure in Figure 1). The east wall is more heavily reinforced and is furthest from the cask drop location. Thus, the floor/wall residual gap is wedge shaped, with the maximum gap on the west wall and essentially zero at the east wall (pivot line). The residual gap predictions for the twelve drop cases and corresponding drop scenarios are listed in Table 2.

Table 2. Residual Floor/Wall Gap Predictions for the Cask Drop Cases and Drop Scenarios

\begin{tabular}{|c|c|c|l|c|c|}
\hline & Drop Case No & $\begin{array}{c}\text { Drop } \\
\text { Scenario }\end{array}$ & $\begin{array}{c}\text { Impact Energy } \\
\text { (ft+lbs) }\end{array}$ & Analysis Category & \multicolumn{2}{|c|}{ Maximum Residual Gap } \\
\hline 1 & 3 & 58,279 & Best Estimate & $<0.001$ & $<0.025$ \\
\hline 2 & 4 & 848,314 & Best Estimate & 0.25 & 6.35 \\
\hline 3 & 4 & 848,314 & Conservative & 0.88 & 22.4 \\
\hline 4 & 5 & $2,006,135$ & Best Estimate & 0.63 & 16.0 \\
\hline 5 & 5 & $2,006,135$ & Conservative & 1.74 & 44.2 \\
\hline 6 & 3 & 58,279 & Conservative & $<0.001$ & $<0.025$ \\
\hline 7 & 1 & $1,095,554$ & Best Estimate & 0.4 & 10.2 \\
\hline 8 & 1 & $1,095,554$ & Conservative & 1.16 & 29.5 \\
\hline 9 & 4 & 115,990 & Best Estimate & $<0.001$ & $<0.025$ \\
\hline 10 & 4 & 115,990 & Conservative & $<0.01$ & $<0.025$ \\
\hline 11 & 2 & 527,160 & Best Estimate & 0.15 & 3.81 \\
\hline 12 & 2 & 527,160 & Conservative & 0.56 & 14.2 \\
\hline
\end{tabular}

\subsection{Impact Energy and Associated Floor/Wall Gap Crack Diameter}

The results of the residual gap predictions for the twelve drop cases listed in Table 2 are graphed in Figure 4 with the residual floor/wall gap diameter as a function of the concrete impact energy for both the best engineering and conservative values of the parameters. The results for each set of data are reasonably linear, specifically in light of the uncertainty associated with the analysis. 
Therefore, each set of data were fit to a linear expression. The fitted linear curves as well as the 5th and 95 th percent confidence levels for the fitted curves are given in Figure 4.

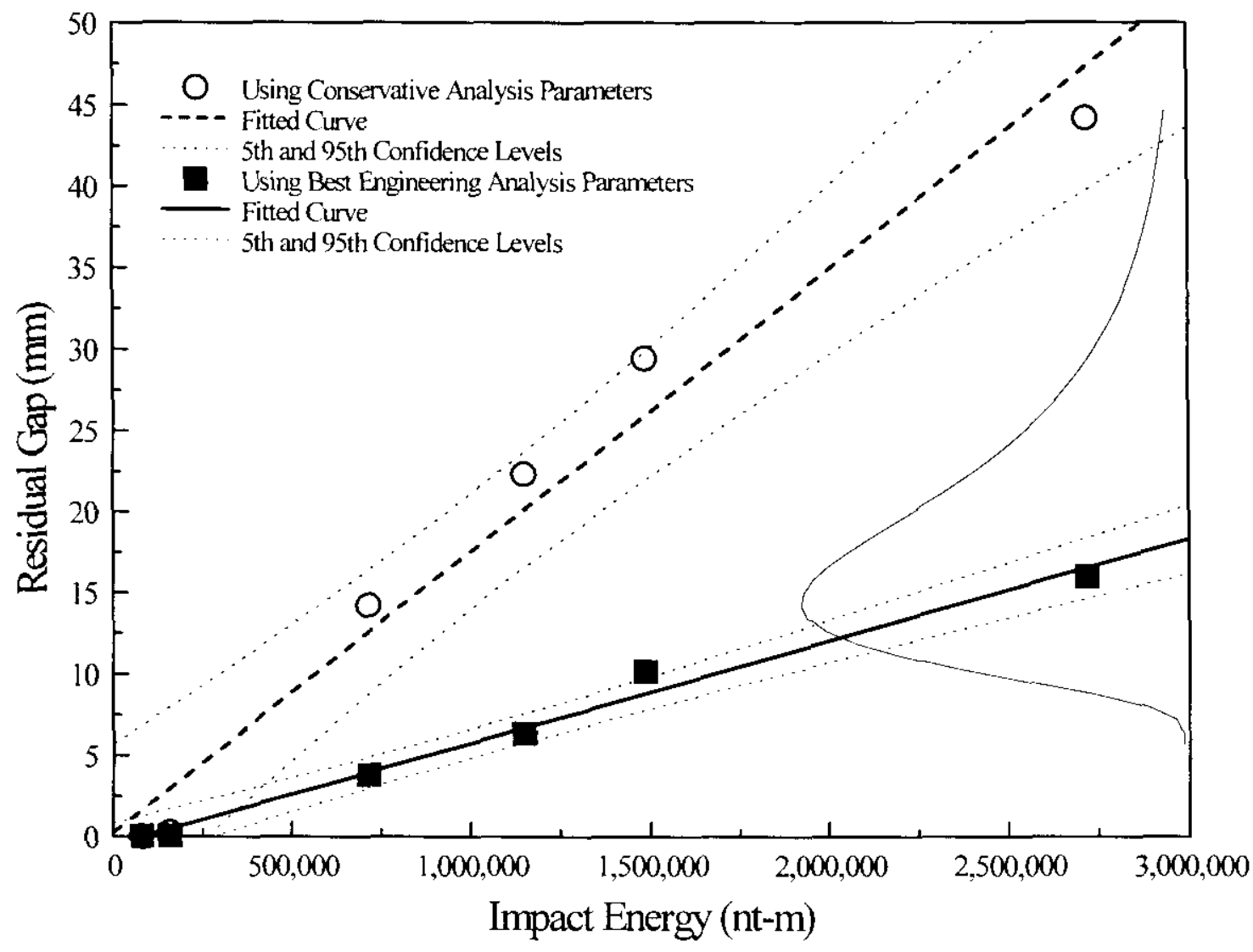

Figure 4. Uncertainty in the Residual Gap as a Function of the Impact Energy.

The uncertainty in the residual floor/wall gap diameter as a function of the impact energy given in Figure 4 was assumed to be lognormaly distributed. The curve representing the results of the analysis using best engineering parameters was assumed to be the median value of the Lognormal distribution. The Lognormal standard deviation was varied until the spread in the distribution enveloped the results of the analysis using the conservative parameters. This process was repeated for each set of data in Figure 4. As the median value of the Lognormal distribution decreased, the spread in the distribution also decreased such that a constant Lognormal standard deviation appeared to be representative of all of the data. Assuming a constant Lognormal 
standard deviation while varying the median value of the Lognormal distribution simplifies the analysis that follows. The results for the distribution of residual floor/wall gap as a function of the impact energy is also shown in Figure 4 as an over-laid Lognormal distribution.

For each value of the concrete impact energy the median value of the distribution for the floor/wall gap is determined using the linear equation that represents the results in Figure 4 using the best engineering parameters. This median value and the constant standard deviation represents a Lognormal distribution from which a value of the floor/wall gap is determined. This process was repeated for 10,000 iterations, each time selecting a new impact energy from the distribution that represents the impact energy (Figure 3). Based on the selected impact energy value, a median value of the Lognormal distribution was selected and from the associated Lognormal distribution for the floor/wall gap, a value for floor/gap was obtained. The results are presented in Figure 5 as the cumulative distribution function which represents the probability of not exceeding a specific floor/wall gap diameter in millimeters. From Figure 5, the most likely floor/wall gap diameter is 0.177 in $(4.5 \mathrm{~mm})$. There is a probability of 0.95 that the floor/wall gap diameter will not exceed 0.472 in $(12 \mathrm{~mm})$. Conversely, there is a probability of 0.05 that the floor/wall gap diameter will be larger than 0.472 in $(12 \mathrm{~mm})$.

\subsection{Flow In A Porous Medium}

The pressure gradient through the floor/wall gap is defined for a porous medium in terms of a viscous pressure drop, an inertial pressure drop, and a pressure drop due to the resistance of the exterior soil (see Appendix D). The expression for the pressure gradient is presented in terms of the superficial velocity and the equivalent hydraulic diameter, from which the superficial velocity is determined. The volumetric flow rate is then equal to the flow area times the superficial velocity. As noted earlier the flow area of the floor/wall gap is a triangular wedge from which the flow area is equal to the SLOP base plus width times the floor/wall gap distance. 


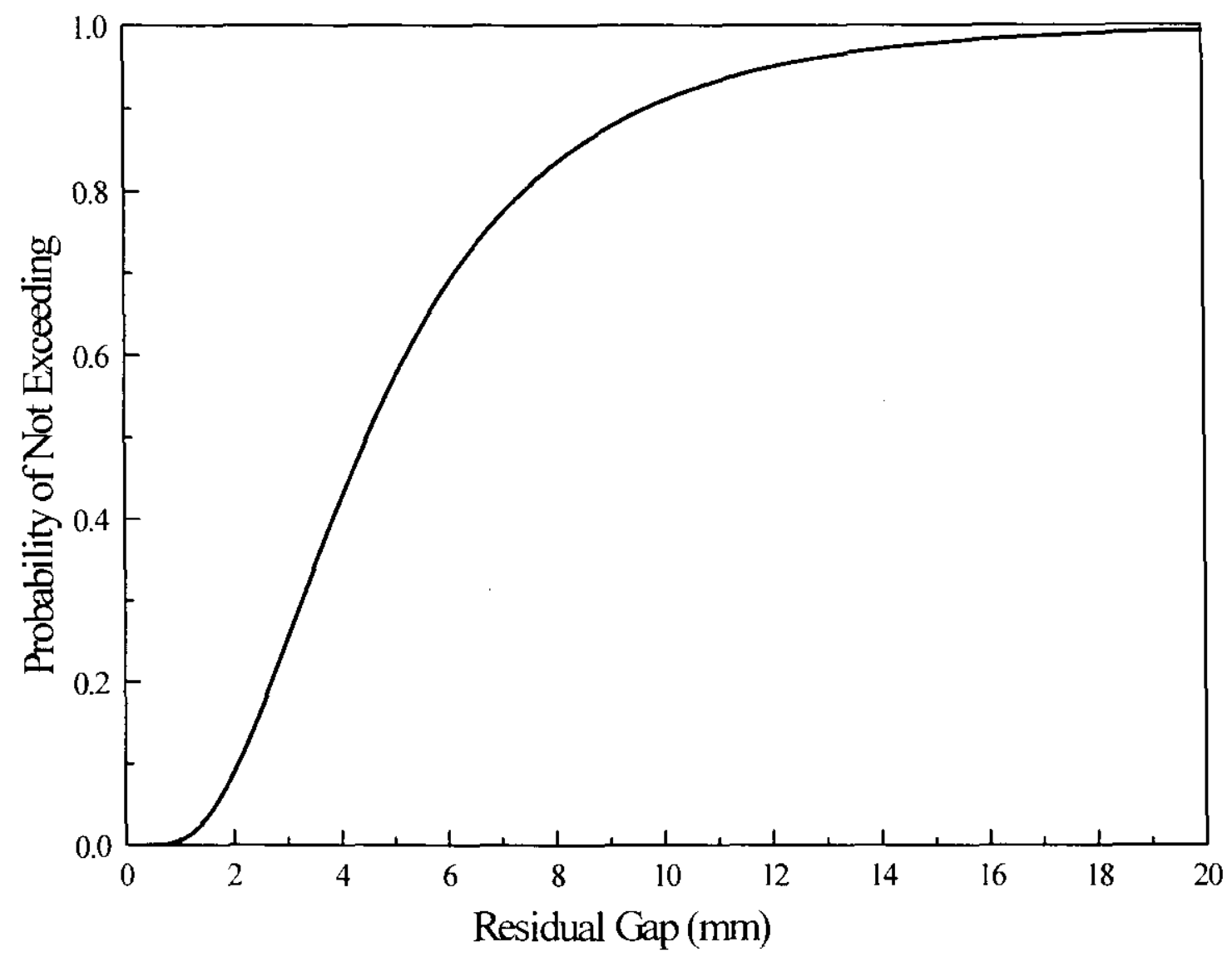

Figure 5. Cumulative Distribution Function of the Residual Floor/Wall Gap.

The contribution due to the soil resistance was not included in the uncertainty analysis because the analysis evaluates the initial flow rate through the floor/wall gap caused by the impact energy of dropping a cask and MCO. The uncertainty analysis does not consider the continuing flow rate that would result from the changing pressure head of the $\mathrm{K}$ Basin water level impeded by soil resistance outside of the basin. See Appendix D for more detailed discussion relative to the contribution to the initial volumetric flow rate from each of the contributions included in the pressure gradient equation. Table 3 provides the volumetric flow rate for flow through a porous medium for various floor/wall gap distances. The parameters used in the analysis are given in Appendix D. 
Table 3. Volumetric Flow Rate for Flow Through a Porous Medium for Various Floor/Wall Gap Distances

\begin{tabular}{|c|c|c|c|c|c|}
\hline \multicolumn{2}{|c|}{$\begin{array}{l}\text { Floor Wall Gap } \\
\text { Distance }\end{array}$} & \multicolumn{2}{|c|}{$\begin{array}{l}\text { Flow Through Porous } \\
\text { Without Soil Resistance }\end{array}$} & \multicolumn{2}{|c|}{$\begin{array}{l}\text { Flow Through Porous Medium } \\
\text { with Soil resistance }\end{array}$} \\
\hline \multicolumn{2}{|c|}{ d } & 0 & 0 & Q & Q \\
\hline$(\mathbf{m m})$ & (in) & $\left(\mathbf{m}^{3} / \mathrm{sec}\right)$ & $(\mathbf{g p m})$ & $\left(m^{3} / \mathrm{sec}\right)$ & (gpm) \\
\hline 0.1 & 0.0039 & $5.1 \times 10^{-5}$ & 0.8 & $4.8 \times 19^{-5}$ & 0.8 \\
\hline 0.2 & 0.0078 & $1.9 \times 10^{-4}$ & 3.0 & $1.6 \times 10^{-4}$ & 2.5 \\
\hline 0.5 & 0.0197 & $8.3 \times 10^{-4}$ & 13 & $4.5 \times 10^{-4}$ & 7.1 \\
\hline 0.6 & 0.0236 & $1.2 \times 10^{-3}$ & 19 & $5.3 \times 10^{-4}$ & 8.3 \\
\hline 1.0 & 0.0394 & $2.4 \times 10^{-3}$ & 38 & $6.1 \times 10^{-4}$ & 9.7 \\
\hline 2.0 & 0.0787 & $6.8 \times 10^{-3}$ & 108 & $6.5 \times 10^{-4}$ & 10.3 \\
\hline 5.0 & 0.1969 & $2.7 \times 10^{-2}$ & 430 & $6.6 \times 10^{-4}$ & 10.4 \\
\hline 10.0 & 0.394 & $7.7 \times 10^{-2}$ & 1217 & $6.6 \times 10^{-4}$ & 10.4 \\
\hline
\end{tabular}

As discussed in more detail in Appendix D, when the soil resistance is included in the pressure drop equation, the volumetric flow rate is substantially reduced for larger floor/wall gap distances. The volumetric flow rate asymptotes to a constant value for floor/wall gap distances larger than $5 \mathrm{~mm}(0.197 \mathrm{in})$. However, the exact contribution to the volumetric flow rate is highly uncertain because the values of the soil permeability around the $\mathrm{K}$ Basin where the leak may occur is uncertain. As noted above, the uncertainty analysis focuses on the initial volumetric flow rate through the floor/wall gap and does not consider the continuing flow rate that would result from the changing pressure head of the $\mathrm{K}$ Basin water level impeded by soil resistance outside of the basin. Therefore, the contribution due to the soil resistance is not included in the following analysis.

\subsection{Contribution Due to the Water Stop}

Figure 2 and the figures in Appendix D identify a water stop located midway in the concrete wall that was used as a flow limiter in fabrication of the floor-wall joint. The primary function of the water stop is to prevent water from escaping though the concrete construction or contraction joint across which the water stop is installed. Appendix D identifies the material of the water stop and its physical specifications.

The water stops in the K Basin SLOP could be damaged or torn by the motion which would separate the concrete floor from the concrete wall. The most likely damage would be vertical 
tears in the water stop as the aged material is stretched and placed in tension. The aged material may not have its initial elongation property ( 450 percent elongation). Further there could be openings created around the bulbs at each end of the water stop around which water could seep.

The contribution of the water stop was represented in the analysis in conjunction with the flow area through the floor/wall gap in terms of the fraction of the flow area that is not available for water flow due to the presence of a portion of the water stop that is intact [i.e., $A=p d(1-F)$ ]. If the fraction of flow area that is not available is one $(F=1)$ then there would be zero flow area that is available for water flow. If the fraction of flow area not available is zero $(F=0)$ then the full flow area would be available for water flow. A uniform probability density function was used to represent the fraction of the flow area that is not available for water flow due to the water stop. The uniform probability density function means that any value of the fraction of area not available for water flow is equally likely.

\subsection{Initial Volumetric Flow Rate}

Each of the conditional probabilities discussed in this section formed a model which was analyzed using Monte Carlo convolution methods. The conditional probabilities that were combined in the final model were: 1) the probability of an impact energy given a load drop, 2) the residual floor/wall gap dependence on the impact energy, 3) the probability of the residual floor/wall gap given an impact energy, and 4) the volumetric flow rate through the floor/wall gap based on flow through a porous medium. Each of the conditional probability distributions were sampled 10,000 times to provide a floor/wall gap distance. Based on the floor/wall gap distance the initial volumetric flow rate was calculated using the equations appropriate for flow in a porous medium. The results were calculated in $\mathrm{m}^{3} / \mathrm{sec}$ but were converted to gallons per minute for presentation of the results.

The probability density function for the distribution for the volumetric flow rate is provided in Figure 6. The corresponding cumulative distribution function is provided in Figure 7. The probability density function that represents the initial volumetric flow rate, for larger flows, is exponentially decreasing. However, the best fit to all of the data is a Lognormal probability density function with log-mean of 3.79 and a log-standard deviation of 1.57 , which relates to a mean of $51.2 \mathrm{gpm}$ and a standard deviation of $29.8 \mathrm{gpm}$. A summary of the results presented in Figures 6 and 7 is given in Table 4. 


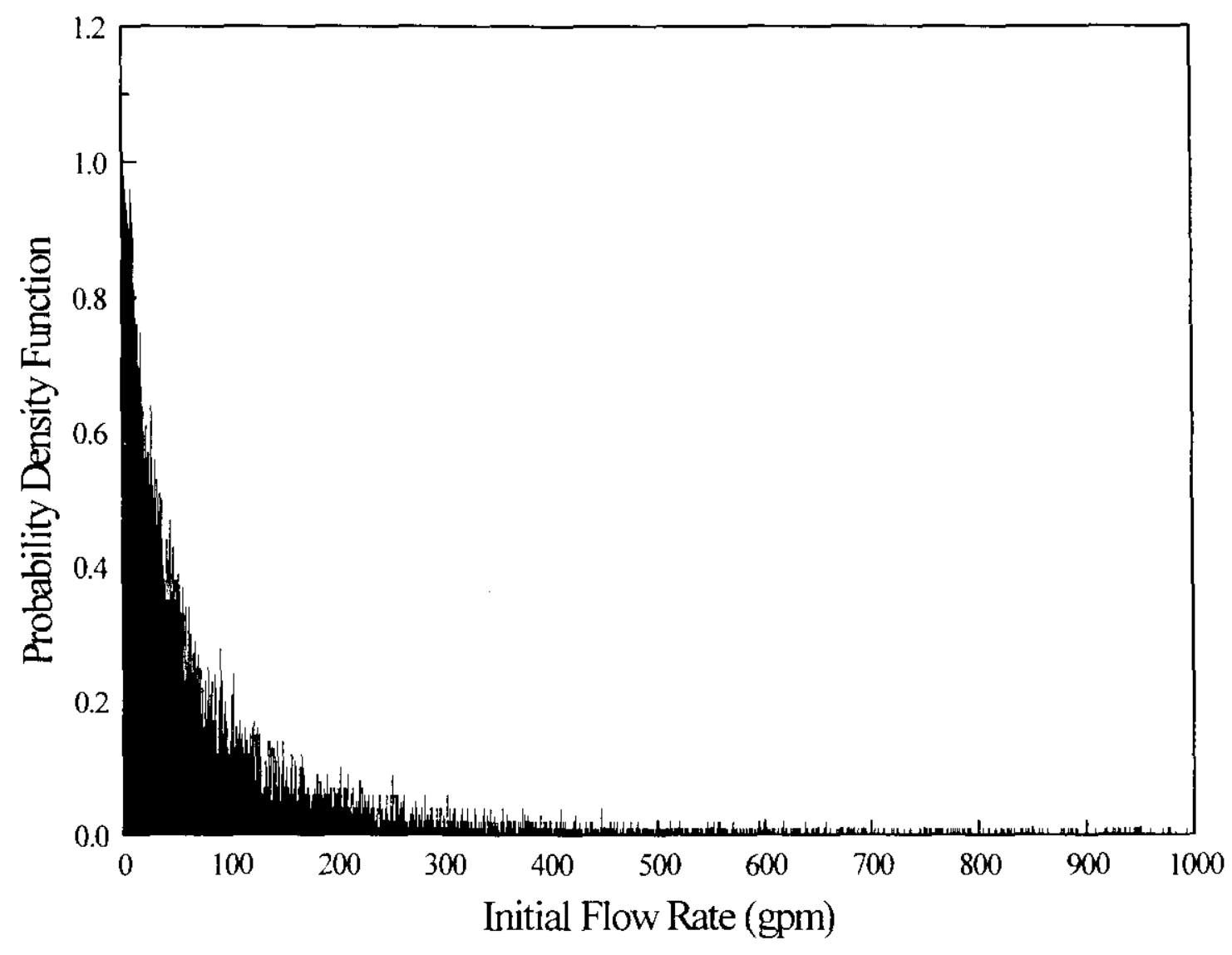

Figure 6. Probability Density Function for the Initial Volumetric Flow Rate. 


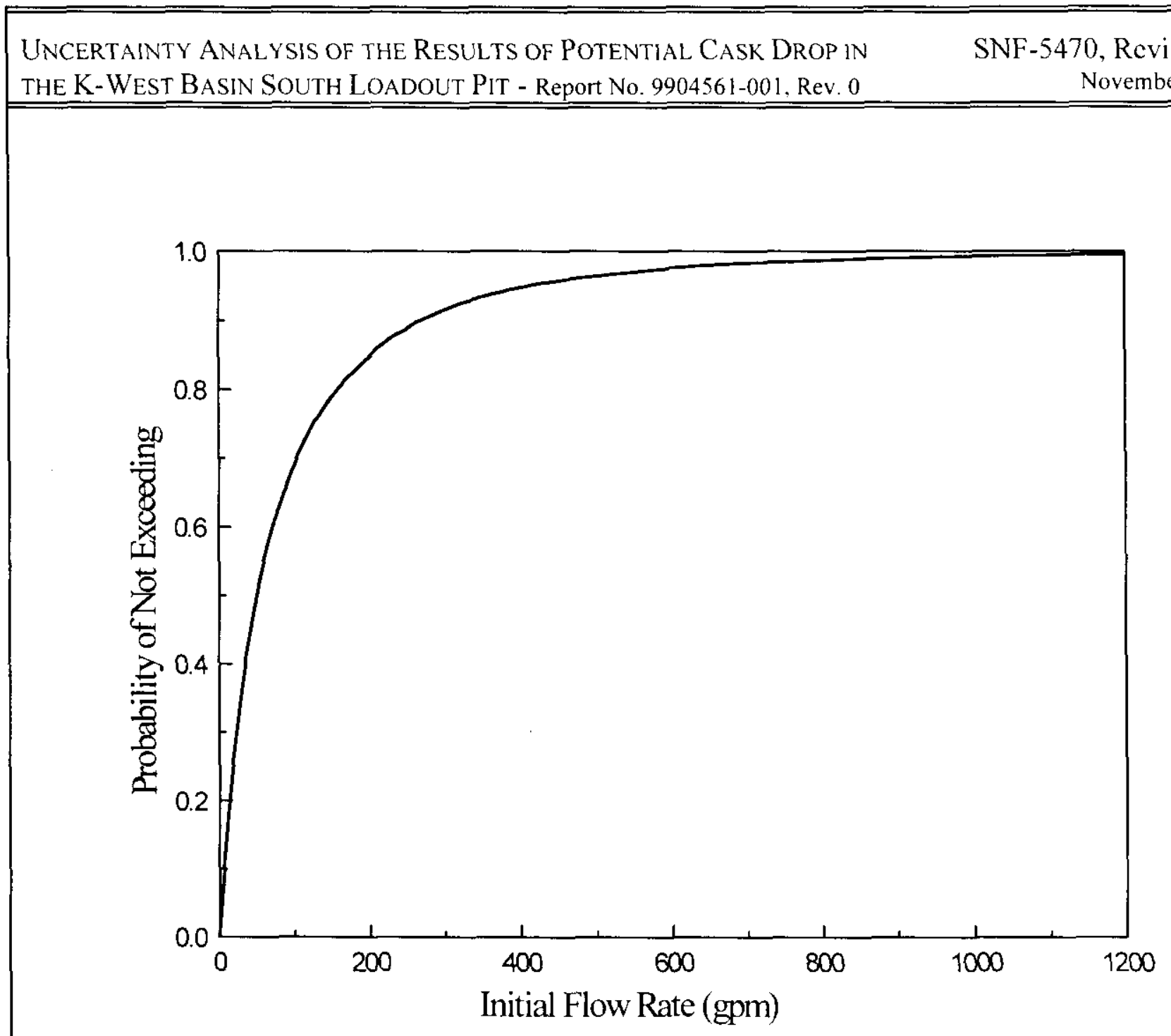

Figure 7. Cumulative Distribution Function for the Initial Volumetric Flow Rate. 
Table 4. Probability of Not Exceeding a Specified Initial Volumetric Flow Rate

\begin{tabular}{|l|c|c|}
\hline 14 & Probability & $\begin{array}{c}\text { Initial Volumetric Flow Rate Is Less } \\
\text { Than (gpm) }\end{array}$ \\
\hline Mean & & 51 \\
\hline 50th Percentile & 0.50 & 50 \\
\hline 90th Percentile & 0.90 & 268 \\
\hline 95th Percentile & 0.95 & 411 \\
\hline 99th Percentile & 0.99 & 916 \\
\hline
\end{tabular}

From Table 4, for example, there is a probability of 0.95 that the volumetric flow rate will be less than $411 \mathrm{gpm}$ given that a cask and MCO are dropped in the K-West Basin SLOP. The associated percentile figures in Table 4 indicate, for example, that 95 percent of the time the volumetric flow rate was less than $411 \mathrm{gpm}$. Stated another way, given the conditional probability that a cask and MCO have been dropped in the K-West Basin SLOP, there is a 95 percent confidence that the resulting initial volumetric flow rate will be less than $411 \mathrm{gpm}$. In the analysis there were a few volumetric flow rates that were as large as $1,000 \mathrm{gpm}$ to $2,000 \mathrm{gpm}$, but these flow rates occurred less than 1 percent of the time. Hence, as noted from Table 4 , 99 percent of the time the volumetric flow rate is less than $916 \mathrm{gpm}$.

A sensitivity analysis was completed to identify the variables that contribute the most to the uncertainty in the initial volumetric flow rate. The results are as expected. The uncertainty in the initial volumetric flow rate is predominantly due to the height at which a cask and MCO may be dropped into the K-Basin SLOP. The uncertainty in the cask drop height is reflected in the uncertainty in the floor/wall gap thickness. Other variables that provide significant contributions to the uncertainty in the initial volumetric flow rate are the effectiveness of the waterstop and the porosity of the concrete rubble in the floor/wall gap. Therefore, as expected, the most effective way to prevent any water flow from the $\mathrm{K}$-Basins is to prevent a cask and $\mathrm{MCO}$ from being dropped. 


\subsection{SUMMARY AND CONCLUSIONS}

The initiating event considered in this uncertainty analysis was the assumption that a load (cask and $\mathrm{MCO}$ ) had been dropped using the transfer bay crane in the K-West Basin SLOP. If a cask and $\mathrm{MCO}$ were to be dropped, the analysis considered the conditional probability of the potential impact energy to the SLOP basin concrete floor. Given the potential impact energy to the basin concrete floor, a distribution was determined for the resultant residual floor/wall gap separation. As a function of the potential floor/wall gap separation, the likelihood of the volumetric flow rate through the floor/wall gap was determined assuming flow through a porous medium.

The results of the uncertainty analysis demonstrated that even if a cask and MCO were to be dropped, the possible initial volumetric flow rate through the opened floor/wall joint would be small. There is a 95 percent confidence (probability of 0.95 ) that, given a load drop, the initial volumetric flow rate would be smaller than $411 \mathrm{gpm}$.

\subsection{REFERENCES}

Ard, K. 1999, Immersion Pail Buoyancy Calculation for the Cask Loadout System, CTFM-M-A.5/A.6-0002, Revision 0.

Brisbin, S., 1997, Design Analysis Report for the TN-WHC Cask and Transportation System, HNF-SD-SNF-FDR-003, Revision 0, Duke Engineering and Services Hanford Inc., Richland, Washington.

Islam, M., 1999, ECN 655698, Supporting Document HNF-SD-SNF-FDR-003, Revision 0.

Kanjilal, S. 1999, 105-K Basins South Loadout Pit Concrete Results for Dropped MCO/Cask, SNF-4345, Revision 0, Duke Engineering and Services Hanford Inc., Richland, Washington.

Kee, A. et..al, 1997, Analysis of Leakage from KE Fuel Storage Basin, HNF-SD-SNF-ANAL017, Revision 0, Duke Engineering and Services Hanford Inc., Richland, Washington.

Kellam, B., 1959, Water Stops For Joints In Concrete, Journal of the American Concrete Institute, June 1959, Detroit, Michigan.

Khaleel, R, and Freeman, E.J., 1995, Variability and Scaling of Hydraulic Properties for 200 Area Soils, Hanford Site, WHC-EP-0883, Westinghouse Hanford Company, Richland. Washington. 
Langevin, A. 1999, Conceptual Design Report - Cask Loadout System/Cask Drop Redesign for the Immersion Pail Support Structure and Operator Interface Platform, $105 \mathrm{~K}$-West Basin, HNF-4576, Revision 0, Flour Daniel Hanford, Richland, Washington.

Macdonald, F. et. al, 1979, Flow Through Porous Media - the Ergun Equation Revisited, Ind. Chem. Fundam.

Moore, C. 1997, K-Basins Immersion Pail Support Structure Drop into the Loadout Pit, HNF-SD-SNF-NAAL-022, Revisions 0 and 0A, Duke Engineering and Services Hanford Inc., Richland, Washington.

Plys, M. 1999, Estimated Leakage Rate through a Cracked Loadout Pit Floor Joint, Fauske \& Associates Memo.

Rizkalla, S. et. al, 1979, Leakage Tests of Wall Segments of Reactor Containments, Technical Report of the Atomic Energy Control Board Nuclear Plant Licensing Directorate.

Wagenblast, G. 1999, Independent Review of Cask Drop Analysis, Design Review Report.

Weamer, J. L., 1999, Justification of Maximum Thickness Crushable Pad, SNFP Interoffice Correspondence. 


\section{Appendix A}

Impact Energy Distribution for Cask and MCO Drops 


\section{A.1 Introduction}

This appendix calculates the distribution for the impact energy that could be experienced by the concrete at the bottom of the K-West Basin SLOP should a loaded cask be dropped by the transfer bay crane. To evaluate the potential impact sequences, it is necessary to identify the component weights for the cask, $\mathrm{MCO}$, and immersion pail. These component weights are provided in Table A-1 along with several combinations of the weights. These weights were identified in a Type II Calculation (Ard 1999) and as noted were obtained from various SNF project literature, including NAC International Drawings and HNF-SD-SNF-FDR-003 (Brisbin 1997). It is noted that various component weights exist in the available SNF project literature which are slightly different from those provided in Table A-1. However, the differences are usually small and the component weights provided in Table A-1 appear to be from the latest design media.

Table A-1. Cask, MCO, Immersion Pail Component Weights

\begin{tabular}{|l|c|c|}
\hline Component Weights._veight (lb) & Mass (kg) \\
\hline Empty MCO w/ Locking Ring & 2400 & 1089 \\
\hline Cask and Cask Lid & 38178 & 17317 \\
\hline Loaded MCO, wet & 20457 & 9279 \\
\hline MCO/Cask Annulus Water & 250 & 113 \\
\hline Immersion Pail, Plug \& Lid & 6356 & 2883 \\
\hline Pail Slings \& Lift Beam & 950 & 431 \\
\hline Total Full Load & 66191 & 30024 \\
\hline System Weights (Combinations) & 40578 & 18406 \\
\hline Cask and Empty MCO & 46934 & 21289 \\
\hline Cask, Empty MCO, \& Immersion Pail & 47884 & 21720 \\
\hline Cask, Empty MCO, Immersion Pail \& Slings & 58885 & 26710 \\
\hline Cask, Loaded MCO \& Annulus Water & 65241 & 29593 \\
\hline Cask, Loaded MCO, Annulus Water \& Immersion Pail & 66191 & 30024 \\
\hline Cask, Loaded MCO, Annulus Water, Immersion Pail \& Slings & & \\
\hline
\end{tabular}

It should be specifically noted that the weights provided in Table A-1 are close but not identical to the weights listed in Table 1-1 of Appendix B. The input for the finite element analysis in Appendix $B$ was the impact energy and the corresponding impact velocity. The impact energy corresponds to the weights of the objects given in Table 1-1 of Appendix B for the various 
vertical drops scenarios defined later in this appendix. The intent in Appendix B was to provide a large distribution of impact energies for which a large distribution of floor/wall residual gap dimensions could be developed.

\section{A.2 Falling Mass Velocity and Kinetic Energy Calculation}

Consider an object of mass $\mathrm{m}$ falling a distance $\mathrm{z}$ through a fluid. The object will experience $\mathrm{a}$ gravitational force, a drag force and a buoyant force, defined by;

$$
\overrightarrow{\mathrm{F}}_{\mathrm{T}}=\overrightarrow{\mathrm{F}}_{\mathrm{g}}+\overrightarrow{\mathrm{F}}_{\mathrm{D}}+\overrightarrow{\mathrm{F}}_{\mathrm{B}}
$$

The gravitational force is given by;

$$
\mathrm{F}_{\mathrm{g}}=-\mathrm{mg},
$$

where $\mathrm{m}$ is the mass of the object, and $\mathrm{g}$ is the gravitational constant.

The buoyant force is equal to the weight of the fluid displaced and is given by;

$$
\mathrm{F}_{\mathrm{B}}=\text { weight }=\mathrm{mg}=\rho \mathrm{gV}_{\text {disp }},
$$

where $\rho$ is the density of the fluid and $\mathrm{V}_{\text {disp }}$ is the volume of the fluid that the object displaces.

The drag force is given by;

$$
\mathrm{F}_{\mathrm{D}}=\frac{1}{2} \mathrm{C}_{\mathrm{D}} \rho_{\text {liquid }} \mathrm{A}_{\mathrm{Fr}} \mathrm{v}^{2}=\mathrm{k} \mathrm{v}^{2} \text {, }
$$

where $C_{D}$ is the drag coefficient, $A_{\mathrm{Fr}}$ is the frontal surface area of the object, $v$ is the velocity of the object, and $\mathrm{k}$ is defined by;

$$
k=\frac{1}{2} C_{D} \rho A_{\mathrm{Fr}} .
$$

The forces act on the center of mass of the object as depicted in the sketch below.

For the falling object, Newton's second law of motion becomes;

$$
\mathrm{m} \frac{\mathrm{dv}}{\mathrm{dt}}=-\mathrm{mg}+\rho \mathrm{g} \mathrm{V}_{\text {disp }}+\mathrm{kv}^{2} \text {. }
$$


The acceleration term in Equation A-6 is expanded as follows;

$$
\frac{\mathrm{dv}}{\mathrm{dt}}=\frac{\mathrm{dv}}{\mathrm{dz}} \frac{\mathrm{dz}}{\mathrm{dt}}=\mathrm{v} \frac{\mathrm{dv}}{\mathrm{dz}}
$$

Rearranging the terms in Equation A- 6 and the expanded form of the acceleration term, Equation A-6 becomes;

$$
\frac{m v d v}{\left(k v^{2}+\rho g v_{D}-m g\right)}=d z
$$

Integrating the above expression gives;

$$
\int_{h}^{z} d z=\int_{v_{0}}^{v_{z}}\left(\frac{m v}{k v^{2}+\rho g v_{D}-m g}\right) d v .
$$

Substituting variables the integral is of the form;

$$
\int_{a}^{b} \frac{d x}{x}=\ln (a)-\ln (b)=\ln \left(\frac{a}{b}\right) \text {. }
$$

Therefore,

$$
z-h=\frac{m}{2 k} \ln \left(\frac{k v^{2}+\rho g V_{D}-m g}{k v_{0}^{2}+\rho g V_{D}-m g}\right)
$$


Taking the exponent of each side of Equation A-11 gives;

$$
\mathrm{e}^{-\frac{2 \mathrm{k}}{\mathrm{m}(\mathrm{h}-\mathrm{z})}}=\left(\frac{\mathrm{kv}^{2}+\rho g \mathrm{~V}_{\mathrm{D}}-\mathrm{mg}}{\mathrm{kv}_{\mathrm{o}}^{2}+\rho \mathrm{gV_{D }}-\mathrm{mg}}\right) \text {. }
$$

Rearranging the terms in Equation A-12 gives the following equation;

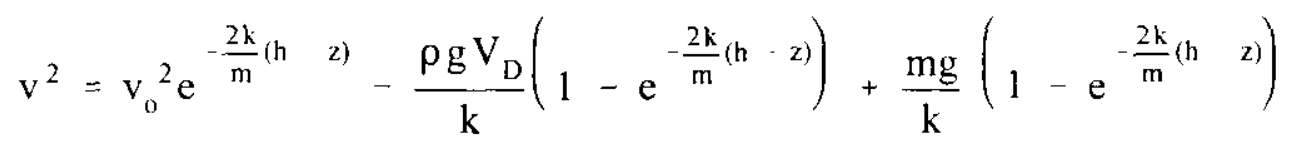

The velocity of the falling object in a fluid is, therefore, given by the equation;

$$
v=\sqrt{v_{0}^{2} e^{\frac{2 k}{m}(h-z)}-\frac{\rho g V_{D}}{k}\left(1-e^{-\frac{2 k}{m}(h-z)}\right)+\frac{m g}{k}\left(1-e^{-\frac{2 k}{m}(h-z)}\right)} .
$$

$\mathrm{v}=$ the objects velocity at any value of $\mathrm{z}<\mathrm{h}$,

$\mathrm{v}_{\mathrm{o}}=$ the objects initial velocity at $\mathrm{z}=\mathrm{h}$,

$$
\mathrm{k}=\frac{1}{2} \mathrm{C}_{\mathrm{D}} \rho \mathrm{A}_{\mathrm{Fr}},
$$

$\mathrm{C}_{\mathrm{D}}=$ the drag coefficient,

$\rho=$ the density of the fluid,

$A_{\mathrm{fr}}=$ the frontal area of the object falling through the fluid,

$\mathrm{g}=$ the gravitation constant,

$\mathrm{h}=$ the initial height of the object,

$\mathrm{m}=$ the mass of the object, and

$V_{D}=$ the volume of the object.

Note that when $z=h$, at the point where the object begins to fall, $v=v_{0}$.

If the drag force is neglected, Equation A-9 becomes;

$$
\int_{h}^{z} d z=\left(\frac{m}{\rho g V_{D}-m g}\right) \int_{v_{0}}^{v_{z}} v d v
$$

or, 


$$
\begin{aligned}
& \frac{2}{m}\left(\rho g V_{D}-m g\right)(z-h)=v^{2}-v_{o}^{2} \\
& v=\sqrt{v_{0}^{2}+\frac{2}{m}\left(m g-\rho g V_{D}\right)(h-z)}
\end{aligned}
$$

If the buoyance force is neglected, Equation A-14 becomes;

$$
v=\sqrt{v_{0}{ }^{2} e^{-\frac{2 k}{m}(h-z)}+\frac{m g}{k}\left(1-e^{-\frac{2 k}{m}(h-z)}\right)} .
$$

If the drag force and the buoyancy force are neglected, the velocity is given by;

$$
\mathrm{v}=\sqrt{\mathrm{v}_{\mathrm{o}}^{2}+2 \mathrm{~g}(\mathrm{~h}-\mathrm{z})} .
$$

The kinetic energy of the object at any time is given by;

$$
E_{\mathrm{KE}}=\frac{1}{2} \mathrm{mv} \mathrm{v}^{2} \text {. }
$$

The force at impact is then given by;

$$
\mathrm{F}_{\text {impact }}=-\mathrm{mg}+\rho \mathrm{gV_{ \text {disp } }}+\mathrm{kv}^{2}{ }_{\text {impact }}
$$

\section{A.3 Potential Cask / MCO Drop Configurations}

As the $\mathrm{K}$-West Basin transfer bay crane lifts a cask and $\mathrm{MCO}$, there are several situations for which a potential vertical cask drop could occur. These potential vertical cask drop situations are illustrated in Figures A-1, A-2 and A-3. A discussion of each potential vertical cast drop scenario follows the appropriate figures. The applicable dimensions though which the cask and MCO could be dropped are also provided on each figure. 


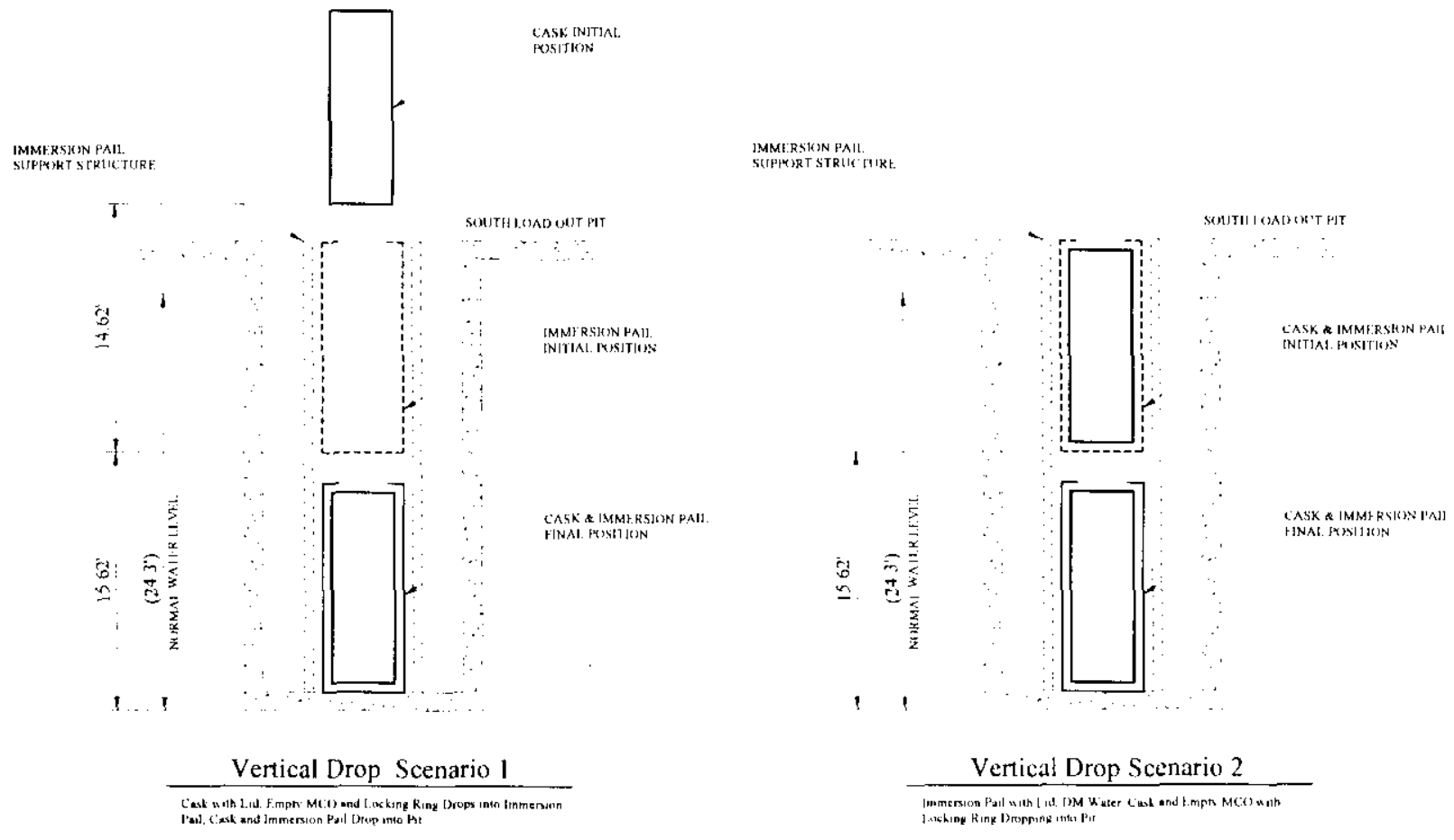

Figure A-1. Vertical Drop Scenarios 1 and 2.

\section{Vertical Drop Scenario 1}

A cask with an empty MCO is lifted from the transport trailer and positioned over the immersion pail support structure in the K-West Basin SLOP. The cask is at a nominal transfer bay crane height. The cask and empty MCO are assumed to drop, impacting the immersion pail. The cask, empty MCO and immersion pail fall and impact the concrete at the bottom of the SLOP.

\section{Vertical Drop Scenario 2}

The cask and empty MCO are situated in the immersion pail. The entire system is lifted by the transfer bay crane and the pins are removed that hold the immersion pail in place. The entire system is assumed to drop such that the cask, empty MCO and immersion pail impact the concrete at the bottom of the SLOP. 

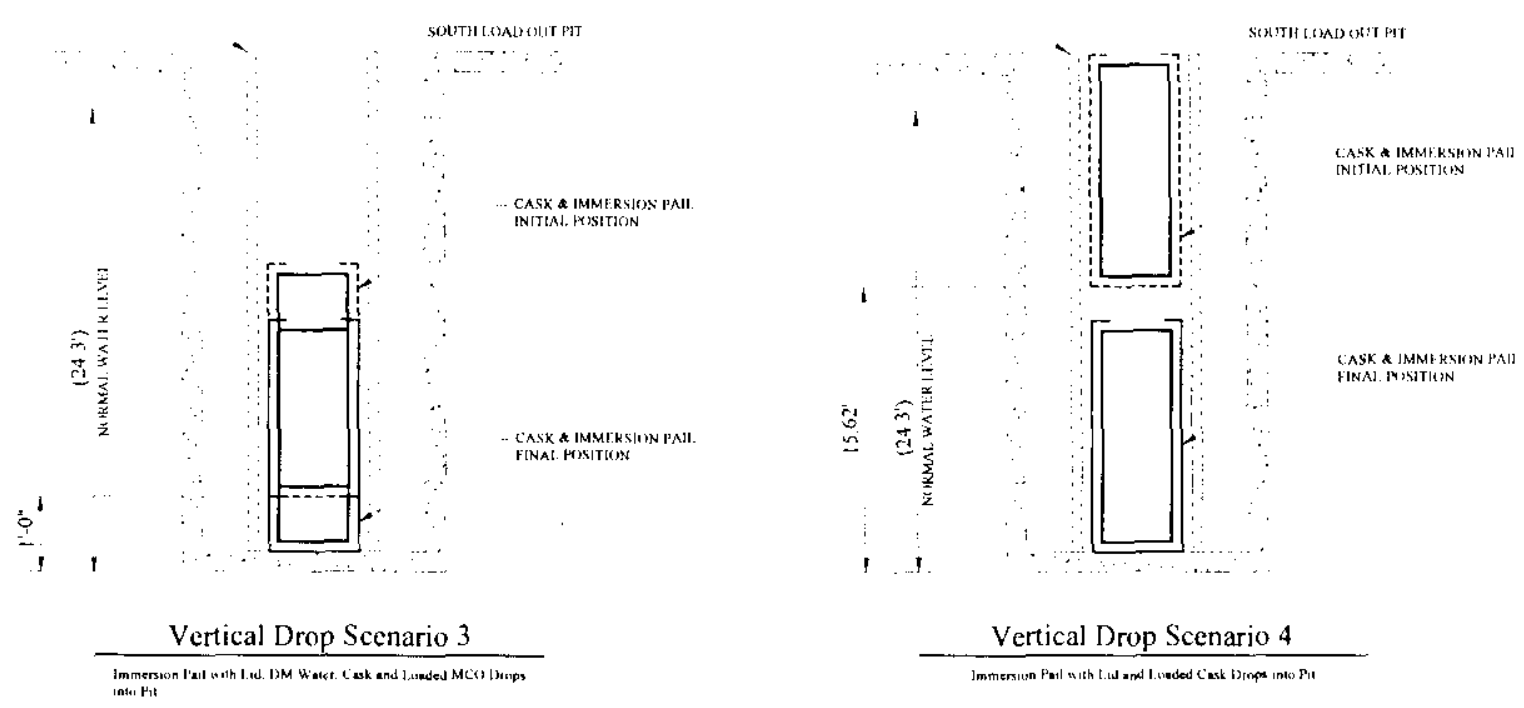

Figure A-2. Vertical Drop Scenarios 3 and 4.

\section{Vertical Drop Scenario 3}

The cask, loaded MCO, and immersion pail are lifted from the bottom of the immersion pail support structure. The cask, loaded MCO and immersion pail are assumed to fall a few feet ( 1 to 2 feet) and impact the concrete at the bottom of the SLOP.

\section{Vertical Drop Scenario 4}

The cask, loaded MCO and immersion pail are lifted to the top of the immersion pail support structure. However, before the pins can be placed to secure the immersion pail, the cask, loaded MCO and immersion pail are assumed to be dropped. The cask, loaded MCO and immersion pail fall and impact the concrete at the bottom of the SLOP. 


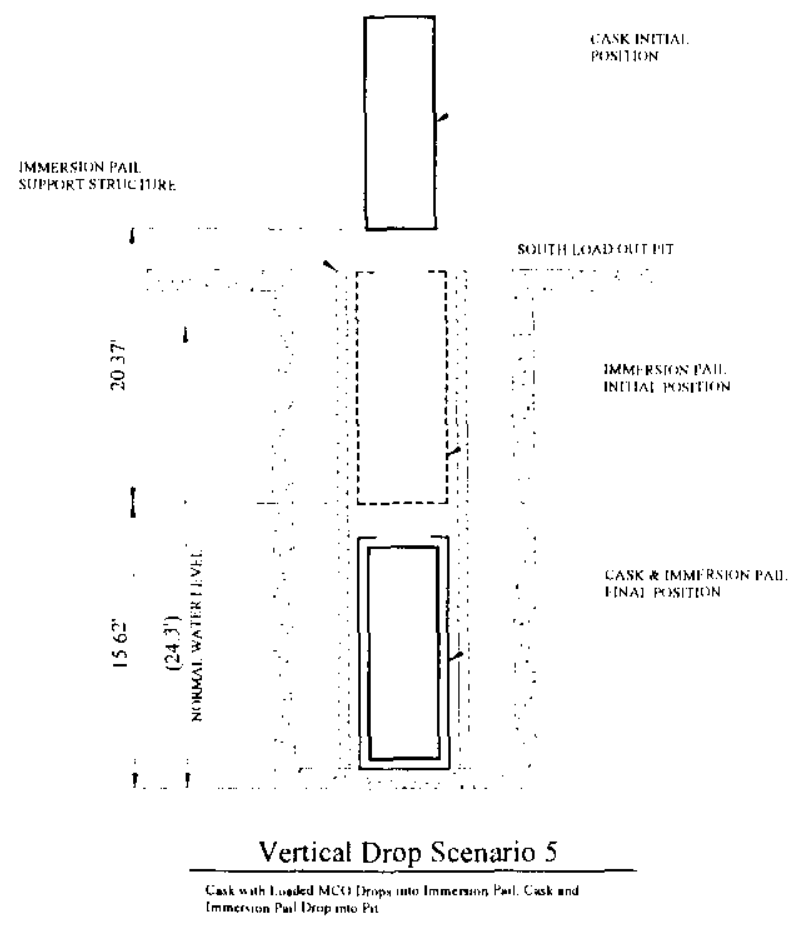

Figure A-3. Vertical Drop Scenario 5.

\section{Vertical Drop Scenario 5}

The cask and loaded MCO are removed from the immersion pail and lifted by the transfer bay crane. A "two-blocking" event or load hangup event are assumed to occur. Thus, the cask and loaded MCO are assumed to be dropped from the nominal transfer bay crane height, impacting the immersion pail. The cask, loaded MCO and immersion pail fall and impact the concrete at the bottom of the SLOP.

\section{A.4 Immersion Pail Design}

A schematic of the immersion pail design is shown in Figure A-4. Foam is attached to the lower two-thirds of the outside of the immersion pail which provides an additional buoyant force. This additional buoyant force reduces the net load of the cask, loaded MCO, and immersion pail such the load required by the transfer bay crane is less that the design rating of 32 tons $(64,000 \mathrm{lb}$.). Note from Table A-1 that the cask, loaded MCO, annulus water, immersion pail and slings weigh 66,191 pounds. However, the buoyant force of the immersion pail decreases the required load by approximately 8,600 pounds, and the added foam further increases the buoyant force by another 
1,800 pounds. An additional design being considered for the immersion pail is to add five to six inches of foam to the bottom of the immersion pail. This additional five to six inches of foam would act as an impact limiter in the event of an immersion pail drop. The five to six inches of foam would also add an additional buoyant force (approximately 400 pounds). The design of the immersion pail shown in Figure A-4 together with the five to six inches of foam at the bottom of the immersion pail were taken into account in the impact energy calculations.
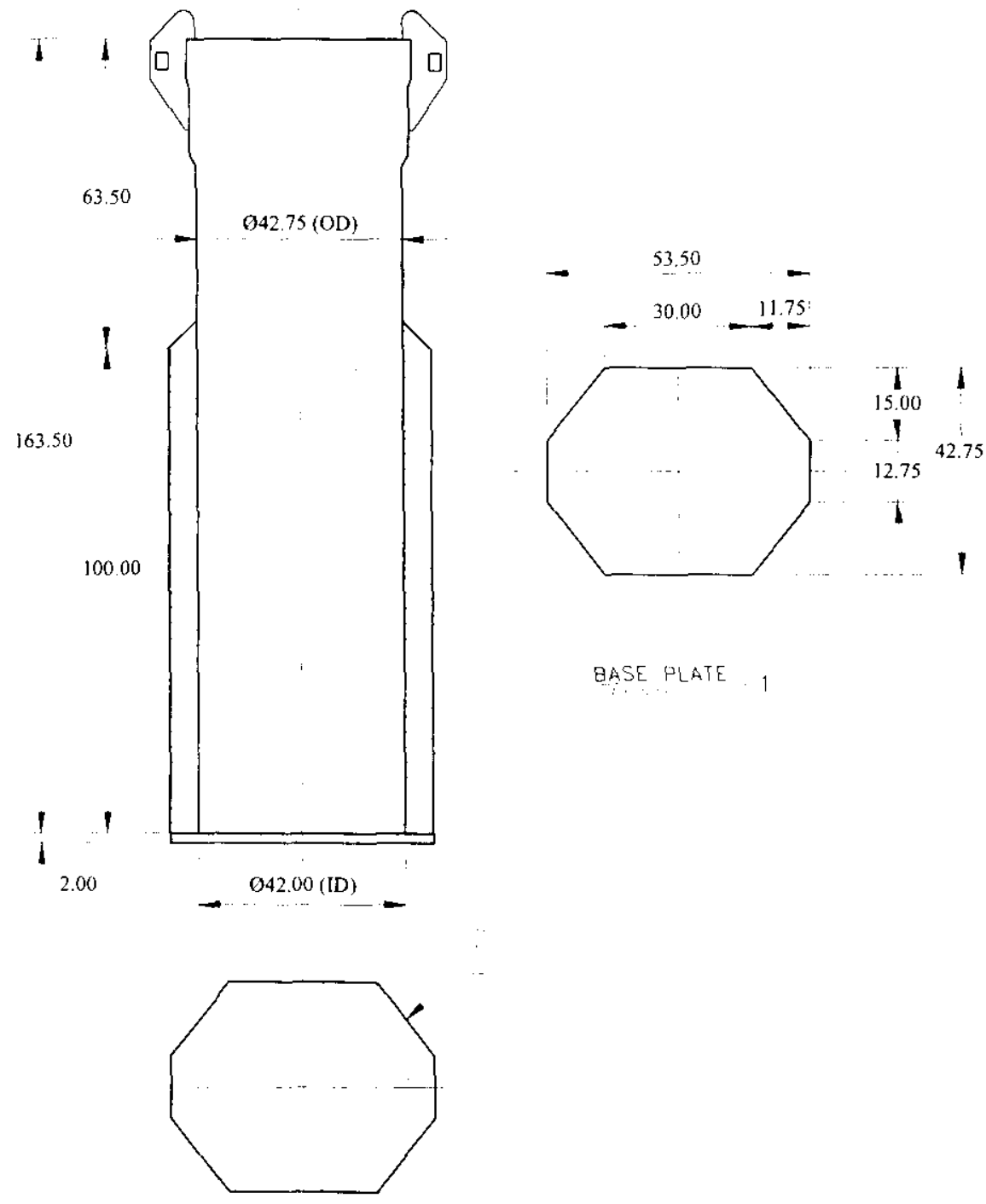

IMNAERSION PAIL

Figure A-4. Schematic of the Immersion Pail and Associated Surface Foam. 


\section{A.5 Cask Drop Results Including Drag and Buoyant Forces}

Using the component weights (Table A-1) for the cask, $\mathrm{MCO}$, and immersion pail, the velocity at concrete impact was calculated including the buoyant and drag forces using Equations A-14, A17 and A-18. The analysis was completed for each of the five vertical drop scenarios. When the system first drops in air and then in water, the equations were solved twice, once to evaluate the drop in air and the second to evaluate the continuing drop in water. The final velocity of the first drop was used as the initial velocity for the second drop. An example for Vertical Drop Scenario 5 is presented in Figure A-5 where the velocity is graphed as a function of the height.

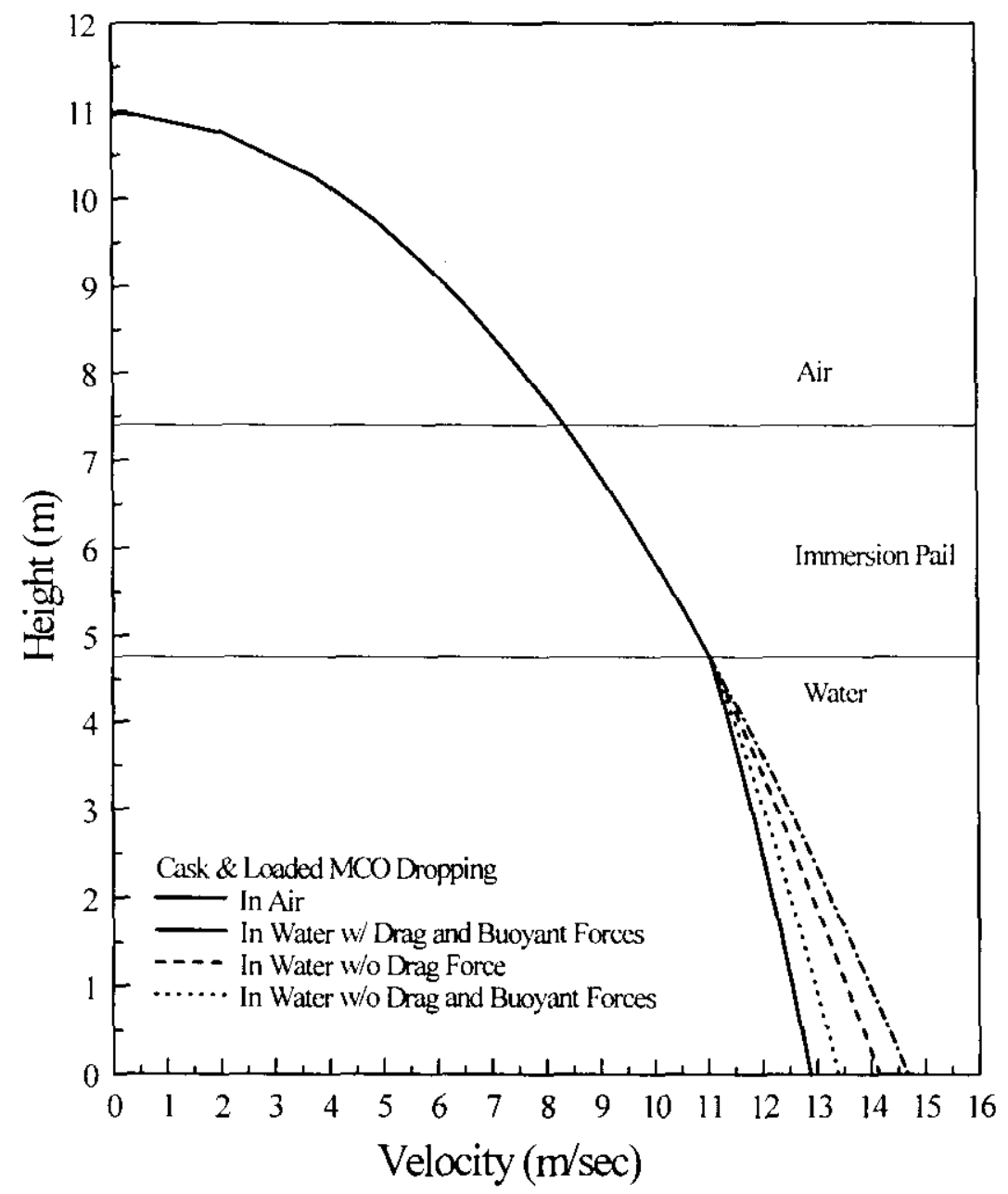

Figure A-5. Velocity of a Dropped Cask, Loaded MCO and Immersion Pail from the "Two-Blocking" Height. 
A summary of the results in Figure A-5 are presented in Table A-2. The contribution in decreasing the velocity at impact, and, hence, the impact kinetic energy is illustrated in Table $A$ 2. Note that in Figure A-5 and Table A-2 no energy was assumed to be lost due to the cask and loaded MCO impacting the immersion pail and shearing the pins which secure the immersion pail. Further, at this stage, no energy was assumed to be lost due to the foam impact limiter attached to the bottom of the immersion pail, nor the impact with the bottom of the immersion pail support structure. These effects are, however, discussed later and are considered in the uncertainty analysis. Also note that both the SI and English units are provided in Table A-2 for the impact velocity and the impact kinetic energy.

Table A-2. Summary Results for Vertical Drop Scenario 5

\begin{tabular}{|c|c|c|c|}
\hline \multicolumn{4}{|c|}{ Impact V elocity ( $\mathrm{m} / \mathrm{sec} / \mathrm{ft} / \mathrm{sec}$ ) } \\
\hline $\begin{array}{l}\text { Without Buoyant } 8 \\
\text { Drag Forces }\end{array}$ & $\begin{array}{l}\text { Without Buoyant } \\
\text { Force }\end{array}$ & Without Drag Force & $\begin{array}{l}\text { With Buoyant \& } \\
\text { Drag Forces }\end{array}$ \\
\hline $14.67 / 48.1$ & $13.39 / 43.9$ & $14.14 / 46.4$ & $12.87 / 42.2$ \\
\hline \multicolumn{4}{|c|}{ Impact Kinetic Energy $(\mathrm{nt}-\mathrm{m} / \mathrm{ft}-\mathrm{b})$} \\
\hline $3.2 \times 10^{6} / 2.4 \times 10^{6}$ & $2.7 \times 10^{6} / 2.0 \times 10^{6}$ & $3.0 \times 10^{6} / 2.2 \times 10^{6}$ & $2.5 \times 10^{6} / 1.8 \times 10^{6}$ \\
\hline
\end{tabular}

A cask drop analysis was completed for each of the vertical drop scenarios identified above. As noted earlier, for those vertical drop scenarios where a cask and loaded MCO, or a cask and empty MCO, were dropped in air (Scenarios 1 and 5), the equations for the velocity were solved twice; first, for the drop in air until the cask bottom contacted the bottom of the immersion pail and, second, for the system dropping in water. The final velocity of the first drop was used as the initial velocity for the second drop (see Equation A-14). However, the energy that may be lost due to the cask and MCO impacting the immersion pail and shearing the pins that secure the immersion pail to the immersion pail support structure was considered in the analysis. This energy to shear the four pins will result in an impact force which can be described by a change in the system momentum. 
Starting from the fundamental relations;

$$
\begin{aligned}
& \mathrm{F}=\mathrm{m} \frac{\mathrm{dv}}{\mathrm{dt}} \text { and } \mathrm{P}=\mathrm{m} \mathrm{v} \\
& \mathrm{F}=\frac{\mathrm{dP}}{\mathrm{dt}}=\frac{\mathrm{dP}}{\mathrm{dz}} \frac{\mathrm{dz}}{\mathrm{dt}}=\mathrm{v} \frac{\mathrm{dP}}{\mathrm{dz}}
\end{aligned}
$$

and integrating,

$$
\begin{aligned}
& \int F d z=m \int v d v \\
& F \delta=\frac{1}{2} m\left(v_{f}^{2}-v_{i}^{2}\right)=K_{i}-K_{i} \\
& \mathrm{KE}_{\mathrm{f}}=\mathrm{KE}_{\mathrm{i}}-\mathrm{F} \delta=\mathrm{KE}_{\mathrm{i}}-\mathrm{U}_{\text {lost }}
\end{aligned}
$$

such that the change in kinetic energy (KE) is just equal to the energy lost in the impact of the cask and MCO with the immersion pail bottom.

The energy required to shear the pins can be written as;

$$
\mathrm{U}_{\text {shcar }}=\frac{1}{2} \mathrm{~F} \delta=\frac{1}{2} \frac{\mathrm{F}}{\mathrm{A}} \mathrm{A} \delta,
$$

where F/A is the pressure exerted on the pins and $\delta$ is the displacement of the pin during the shear. For the immersion pail there are four lifting lugs through which the locking pins are inserted to secure the immersion pail to the immersion pail support structure. A shear stress for double shear of the locking pins is included in the cask Design Analysis Report (Brisbin 1997). The shear stress (force per unit area) is evaluated to be 49,020 psi with a safety margin to 81,000 psi. The locking pin is a square pin with cross sectional area of 1.5 -in by 1.5 -in. Assuming the entire displacement of 1.5-in for complete shear of the pins gives an energy to shear the pins of approximately $26,600 \mathrm{ft}-\mathrm{lb}$. $(36,000 \mathrm{nt}-\mathrm{m})$ for the maximum 81,000 psi double shear stress. It is understood that this energy to shear the pins is an approximation because shearing of the pins is actually a dynamic situation. Therefore, in the analysis the energy to shear the pins was varied by a triangular probability density function between $0 \mathrm{ft}-\mathrm{lb}(0 \mathrm{nt}-\mathrm{m})$ to $73,889 \mathrm{ft}-\mathrm{lb}(100,000 \mathrm{nt}-\mathrm{m})$ with the most likely energy of $26,600 \mathrm{ft}-1 \mathrm{~b}(36,000 \mathrm{nt}-\mathrm{m})$.

The energy to shear the pins is subtracted from the kinetic energy at impact to determine the kinetic energy after impact. The kinetic energy after impact is used to evaluate the system velocity after impact which is used as the initial velocity for the second part of the drop. 
For those drop scenarios that start with the cask and MCO in the immersion pail, either at the height of the secured immersion pail or lower, only one drop was considered. Equation A-14 was used to evaluate the velocity of the system at the bottom of the drop. The total buoyant force of the immersion pail including the additional volume of the foam around the bottom part of the immersion pail (including the foam at the bottom of the immersion pail) was included in solving Equation A-14.

Credit was taken for the energy absorbed by the impact limiter foam at the bottom of the immersion pail. Credit was also taken for the energy absorbed by the loaded immersion pail striking the bottom of the immersion pail support structure and shearing the threads of the four legs that hold the immersion pail support structure above the concrete floor. The kinetic energy of the system just before hitting the concrete floor of the SLOP was calculated from the velocity of the system just before hitting the concrete floor. This kinetic energy of the system was then reduced by the energy absorbed by the impact limiting foam and the energy absorbed by yielding the bottom of the immersion pail support structure.

The design of the foam impact limiter at the bottom of the immersion pail was predicated on an energy absorption capacity based on a maximum crush force of 450,000 lb (Langevin 1999) and a maximum foam height of five inches (Islam 1999). Based on the foam manufacturer's data, this equates to an average crush strength of $400,580 \mathrm{lb}$ from 10 percent to 70 percent strain. The energy absorbing capacity is taken as the average crush strength times the displacement ( $\mathrm{F} \delta$ ). The nominal energy absorbing capacity of the five-inch foam is $140,202 \mathrm{ft}-\mathrm{lb}(190,000 \mathrm{nt}-\mathrm{m})$. A total displacement of five inches provides a nominal crush strength of $200,000 \mathrm{ft}-\mathrm{lb}(272,000 \mathrm{nt}-$ $\mathrm{m})$ and a minimum 10 percent energy absorbing capacity at five-inches is $177,300 \mathrm{ft}-\mathrm{lb}(240,000$ nt-m). Therefore, the energy absorbed by the impact limiter foam at the bottom of the immersion pail was expressed as a triangular probability density function ranging from $125,100 \mathrm{ft}-\mathrm{lb}$ $(170,000 \mathrm{nt}-\mathrm{m})$ to $199,500 \mathrm{ft}-\mathrm{lb}(270,000 \mathrm{nt}-\mathrm{m})$ with the most likely value of $177,300 \mathrm{ft}-\mathrm{lb}$ $(240,000 \mathrm{nt}-\mathrm{m})$.

The energy absorbed by the loaded immersion pail striking the bottom of the immersion pail support structure and shearing the threads of the four legs was assumed to be similar to the energy absorbed by shearing the locking pins. However, it was realized that there was much greater uncertainty (unknown) in this energy such that a much wider range in the absorbing energy was used. Therefore, the energy absorbed by the bottom of the immersion pail support structure was expressed as a triangular probability density function ranging from $0 \mathrm{ft}-\mathrm{lb}(0 \mathrm{nt}-\mathrm{m})$ to $199,500 \mathrm{ft}-\mathrm{lb}(270,000 \mathrm{nt}-\mathrm{m})$ with the most likely value of $14,800 \mathrm{ft}-\mathrm{lb}(20,000 \mathrm{nt}-\mathrm{m})$.

The other variables for which the uncertainty was expressed as a probability density function include the drag coefficient and the drop height. Literature values for the drag coefficient of a flat plate with the velocity perpendicular to the plate diameter range from a high of 2 to a low of 
0.5 with the best estimate of 1.2 . Therefore, the drag coefficient was expressed as a triangular probability density function ranging from 0.1 to 2.0 with the most likely value of 1.2 .

The drop height was expressed as a uniform probability density function ranging from zero to 30.24 feet (9.2 meters) which is the nominal crane transfer height. That is, it was assumed that a drop from any height was equally possible. A "two-blocking" event, if it were to occur, could conceivably results in a cask drop from 36 feet (11 meters). However, before the cask could reach this height the limit switch would terminate power to the crane motor and prevent further raising of the cask. The likelihood of a "two-blocking" event is reasonably small. Therefore the maximum drop height of a cask and MCO was considered to be the just above the nominal transfer crane height

The weight of the total cask was randomly varied between two states; either a loaded MCO or an empty MCO. If a loaded MCO was chosen, then the mass considered was a cask and loaded MCO for drop heights above the immersion pail. For drop heights below the immersion pail the mass considered was a cask, loaded $\mathrm{MCO}$ and immersion pail. If an empty $\mathrm{MCO}$ was chosen, then the mass considered was a cask and empty MCO for drop heights above the immersion pail and a cask, empty MCO, and immersion pail for drop heights below the immersion pail. The random selection of the two states was very nearly equal to $50: 50$; that is, equally likely to have an empty MCO as a loaded MCO. This is very reasonable because of the four lifts for each MCO half of the lifts will be with a loaded MCO and the other half will be with an empty MCO.

All of the variables were varied between their limits (i.e., described by their probability density function) using a Monte Carlo process. The calculated result was the remaining impact energy that could be imparted to the concrete floor of the SLOP. This process equated to calculating the velocity of the mass before impact taking into account the uncertainty in the drag coefficient, the drop height, and the energy to shear the immersion pail locking pins. The kinetic energy at impact was calculated from the impact velocity. The energy absorbed by the impact limiting foam and the energy absorbed by the loaded immersion pail striking the bottom of the immersion pail support structure was subtracted from the kinetic energy at impact to obtain the available impact energy. The process was repeated 10,000 times. This equates to completing 10,000 what if analyses varying all of the available parameters.

The results of this analysis are given in Figure A- 6 which gives the distribution of data for the impact energy in nt-m that could be imparted to the SLOP concrete floor if a cask were to be dropped. Note that because of the many parameters that are being varied in this analysis, the scatter in the data is more than is usual for an analysis of this type. However, the data were fitted to a probability density function. The probability density function that best fit the data was a Weibull probability density function (solid line in Figure A-6). A Lognormal probability density function did not have an extensive enough tail in the distribution to adequately represent the 
larger impact energies that were observed in the data. The fitted probability density function is used to represent the impact energy from a dropped cask in the remainder of the uncertainty analysis.

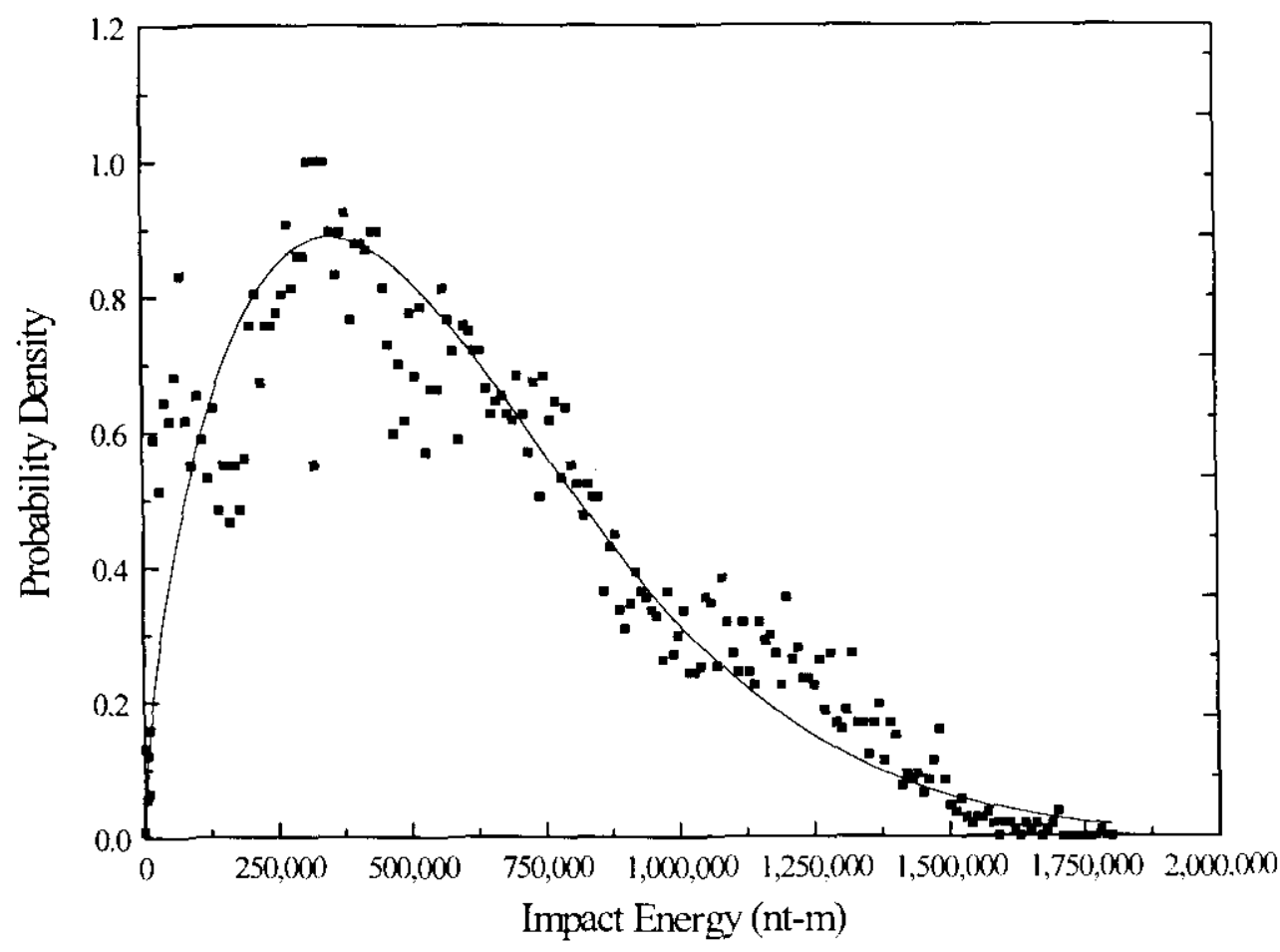

Figure A-6. Probability Distribution for the Impact Energy That Could be Imparted to The SLOP Concrete Floor by a Dropped Cask.

A summary of the results presented in Figure A-6 is provided in Table A-3, which gives the impact energy that could be imparted to the SLOP concrete floor should a cask be dropped by the SLOP transfer bay crane. The parameters that defined the Weibull probability density function that was the best fit to the data (solid line in Figure A-6) are also listed in Table A-3. 
Table A-3. Summary Results of Impact Energy That Could Be Imparted to SLOP Concrete Floor by a Dropped Cask

\begin{tabular}{|c|c|c|}
\hline \multirow[b]{2}{*}{ Percentile } & \multicolumn{2}{|c|}{ Impact Energy Imparted to SL.OP Concrete Floor } \\
\hline & $(\mathrm{nt}-\mathrm{m})$ & $(\mathrm{ft}+\mathrm{b})$ \\
\hline 50 th & 394,400 & 290,700 \\
\hline 90 th & $1,059,690$ & 781,070 \\
\hline 95 th & $1,224,050$ & 902,220 \\
\hline 95 th & $1,448,600$ & $1,067,740$ \\
\hline \multicolumn{3}{|c|}{ Weibull Parameters for Fitted Distribution } \\
\hline Location Parameter & Scale Parameter, $\eta$ & Shape Parameter, $\beta$ \\
\hline 0 & 639,756 nt-m & 1.61 \\
\hline
\end{tabular}




\section{Appendix B \\ K Basin South Load Out Pit Wall-floor Joint Deformation Predictions}




\title{
M \& D
}

Professional Services Inc.

K BASIN SOUTH LOAD OUT PIT

\author{
WALL-FLOOR JOINT DEFORMATION PREDICTIONS \\ FOR VARIOUS CASK DROP ACCIDENTS
}

Prepared by:

M\&D Professional Services, Inc. 1305 Mansfield, Suite 5

Richland, WA 99352

Phone: (509) 943-1625 ext 231

Fax: (509) 946-2213

Prepared for:

ARES Corporation

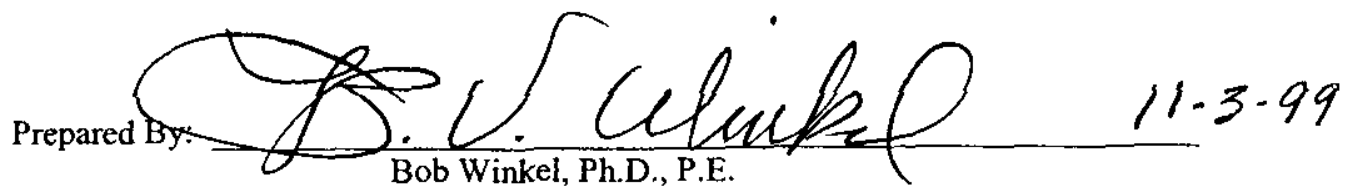




\begin{tabular}{|c|c|c|c|}
\hline & \multirow{2}{*}{\multicolumn{2}{|c|}{ CALCULATION SHEET }} & Page No. \\
\hline Professional & & & 1 \\
\hline \multirow{2}{*}{\multicolumn{2}{|c|}{$\begin{array}{l}\text { Client Location } \\
\text { ARES Corporation }\end{array}$}} & Prepared 8y & $\begin{array}{l}\text { Calculation No. } \\
\text { M\&D 99-013 }\end{array}$ \\
\hline & & B. Winkel & \multirow{3}{*}{$\begin{array}{c}\text { Revision No. } \\
0 \\
\text { Supporting Document }\end{array}$} \\
\hline \multicolumn{2}{|c|}{$\begin{array}{l}\text { Subject } \\
\text { Floor/Wall Joint Evaluation for Drop Loading }\end{array}$} & Checked By & \\
\hline \multicolumn{2}{|c|}{$\begin{array}{l}\text { Ref. Drawing(s) } \\
\mathrm{H}-1-21072\end{array}$} & Revised By / Date & \\
\hline
\end{tabular}

\section{CONTENTS}

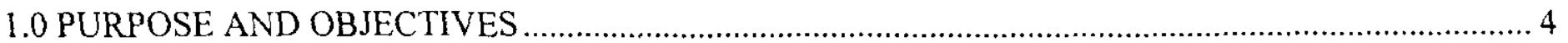

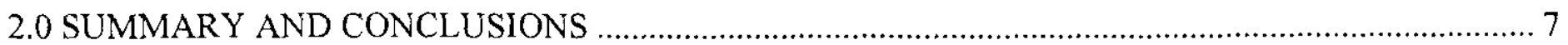

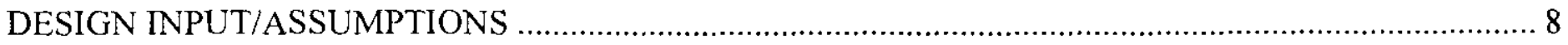

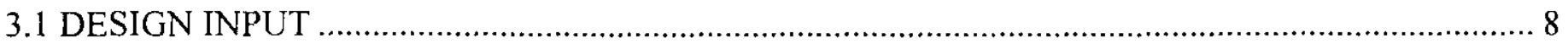

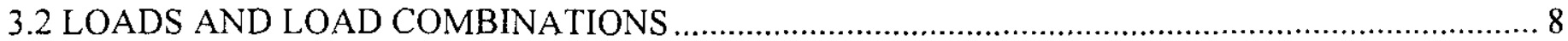

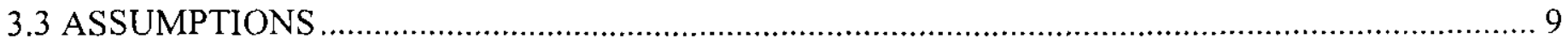

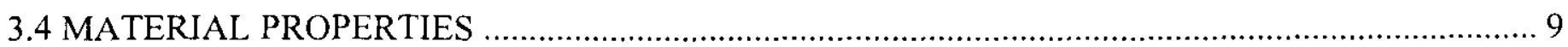

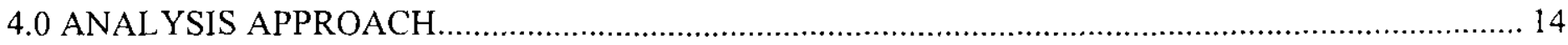

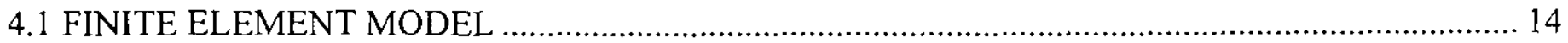

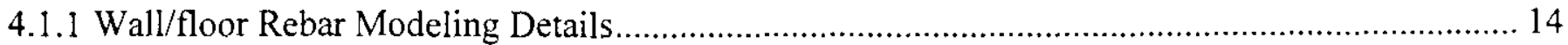

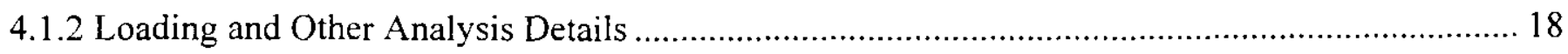

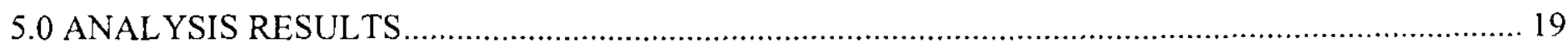

6.0 REFERENCES

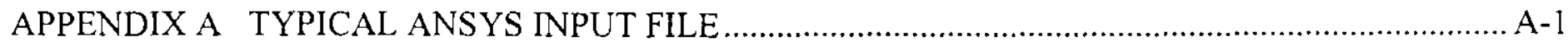

APPENDIX B BENCHMARK TESTING OF ANAL YSIS METHODOLOGY ……............................. B-1 
SNF-5470, Revision 0

\begin{tabular}{|c|c|c|c|}
\hline M\&D ${ }^{\text {Professional }}$ & \multicolumn{2}{|c|}{ CALCULATION SHEET } & \multirow{2}{*}{\begin{tabular}{|cc} 
Page No. & of \\
2 & 21 \\
CalculationNo. \\
M\&D
\end{tabular}} \\
\hline \multicolumn{2}{|l|}{$\begin{array}{l}\text { Creent/location } \\
\text { ARES Corporation }\end{array}$} & $\begin{array}{l}\text { Prepared By } \\
\text { B. Winkel }\end{array}$ & \\
\hline \multicolumn{2}{|c|}{$\begin{array}{l}\text { Subject } \\
\text { Floor/Wall Joint Evaluation for Drop Loading }\end{array}$} & & $\begin{aligned} 0 \\
\end{aligned}$ \\
\hline \multicolumn{2}{|c|}{$\begin{array}{l}\text { Ref. Drawing(s) } \\
\text { H-1-21072 }\end{array}$} & Revised By /Date & \\
\hline
\end{tabular}

\section{FIGURES}

Figure 1-2 - Cask Drop Configuration in South Load Out Pit .................................................................. 6

Figure 1-3 - Impact Limiter Assumed Force/Deflection Properties ................................................................ 7

Figure 2-1 - Maximum Residual Gap Versus Impact Energy..................................................................... 8

Figure 3-1 - Soil Rebound Percentage Versus Maximum Deformation ....................................................... 13

Figure 4-1 - Finite Element Model of the K Basin Wall/Floor Joint .......................................................... 16

Figure 4-2 -- Evaluation of Construction Joint Rebar Model Adequacy ...................................................... 17

Figure 5-1 - Typical Floor Deformation Pattern ..................................................................................... 19 


\begin{tabular}{|c|c|c|c|}
\hline \multicolumn{4}{|c|}{ SNF-5470, Revision 0} \\
\hline & \multicolumn{2}{|c|}{ CALCULATION SHEET } & $\begin{array}{c}\text { Page No. } \\
3\end{array}$ \\
\hline \multirow{2}{*}{\multicolumn{2}{|c|}{$\begin{array}{l}\text { Clent/Location } \\
\text { ARES Corporation }\end{array}$}} & Prepared By & $\begin{array}{l}\text { CalculationNo. } \\
\text { M\&D 99-013 }\end{array}$ \\
\hline & & B. Winkel & Revision No. \\
\hline \multicolumn{2}{|c|}{$\begin{array}{l}\text { Subject } \\
\text { Floor/Wall Joint Evaluation for Drop Loading }\end{array}$} & Checked By & \multirow{2}{*}{$\frac{0}{\text { Supporing Uocumentivo. }}$} \\
\hline \multicolumn{2}{|c|}{$\begin{array}{l}\text { Ret. Drawing(s) } \\
\mathrm{H}-1-21072\end{array}$} & RevisedBy/Date & \\
\hline
\end{tabular}

\section{TABLES}

Table 1-1 - Definitions of Cask Drops into South Load Out Pit................................................................... 5

Table 3-1 - Material Properties Used in the Basin Drop Load Calculations ................................................ 10

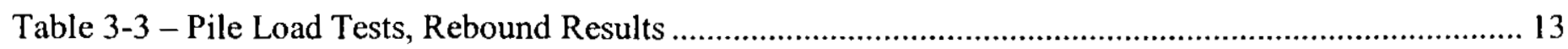

Table 5-1 - Residual Gap Predictions for the Cask Drop Cases ................................................................... 20 
SNF-5470, Revision 0

\begin{tabular}{|c|c|c|c|}
\hline $\mathbf{M} \boldsymbol{k} \mathbf{D}^{\text {Professional }}$ & \multicolumn{2}{|c|}{ CALCULATION SHEET } & \multirow{2}{*}{ 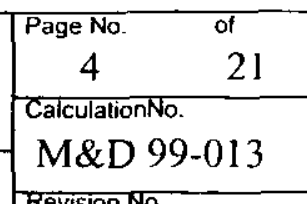 } \\
\hline \multicolumn{2}{|c|}{ Clenthocation } & Prepared 8y & \\
\hline \multicolumn{2}{|c|}{ Subject } & \begin{tabular}{|l|} 
B. Winkel \\
Checked By \\
\end{tabular} & 0 \\
\hline \multicolumn{2}{|c|}{$\begin{array}{l}\text { Ret. Drawing(s) } \\
\text { H-1-21072 }\end{array}$} & Revised By/Date & \\
\hline
\end{tabular}

\section{SOUTH LOAD OUT PIT CASK DROP ANALYSES SUPPORTING PROBABILISTIC RISK ASSESSMENT EVALUATION}

\subsection{PURPOSE AND OBJ ECTIVES}

As input to a probabilistic risk assessment of cask drops into the K Basin South Load Out Pit (SLOP), a series of twelve dynamic analyses of cask drops onto the SLOP floor have been performed. Definitions of the cask drop cases is provided in Table 1-1. Note that drop energies ranging from 58,000 to $2,000,000 \mathrm{ft}-\mathrm{lbs}$ with drop weights ranging from 45,300 (empty MCO) to 66,200 lbs. (loaded MCO) were considered. The objective of each drop analysis is to estimate the peak impact force and the wall/floor joint opening magnitudes resulting from the impact.

The impact load path from the cask to the floor is illustrated in Figure 1-1. The properties of the foam impact limiter shown on the bottom of the immersion pail have not been finalized, but, per current estimates, the impact limiter will have a crushing strength of $450,000 \mathrm{lb}$. and will be 5 -in. thick. The $450,000 \mathrm{lb}$. crushing strength corresponds to the floor "capacity" (zero leakage) reported in Appendix I of Reference 13. Assuming an effective crush magnitude of $50 \%$, the energy absorption capacity of the foam is about $(2.5 / 12)(450,000)=93,800 \mathrm{ft}-\mathrm{lb}$. Thus, for a $66,200 \mathrm{lb}$. cask, a drop height greater than about $(93,800 / 66,200)=1.4 \mathrm{ft}$. will exceed the impact limiter capacity, resulting in a "bottoming out" of the impact limiter, and much higher forces imparted to the SLOP floor. Note that only Case Nos. 1 and 6 have drop energies less than the capacity of the impact limiter.

Based upon prior experience with evaluating drops onto the SLOP floor, the primary leak path following a cask drop, is the construction joint between the SLOP floor and the SLOP walls. Therefore, a finite element model of the lower portion of the SLOP was developed which focused on the wall/floor joint. This finite element model was used for performing dynamic analyses for each of the 12 cask drop cases listed in Table 1-1. The analysis methodology is described in Section 4.0. A summary of the results and the conclusions drawn are provided in Section 2.0. 
SNF-5470, Revision 0

\begin{tabular}{|c|c|c|c|}
\hline $\mathbf{M} \& \mathbf{D}^{\text {Professional }}$ & \multicolumn{2}{|c|}{ CALCULATION SHEET } & \multirow{2}{*}{$\begin{array}{|cc|}\text { Page No. } & \text { of } \\
5 & 21 \\
\text { CalculationNo. } & \\
\text { M\&D 99-013 }\end{array}$} \\
\hline $\begin{array}{l}\text { Clent/Location } \\
\text { ARES Corporation }\end{array}$ & & $\begin{array}{l}\text { Prepared By } \\
\text { B. Winkel }\end{array}$ & \\
\hline $\begin{array}{l}\text { Subject } \\
\text { Floor/Wall Joint Evaluat }\end{array}$ & oading & & 0 \\
\hline $\begin{array}{l}\text { Ref. Drawing(s) } \\
\mathrm{H}-1-21072\end{array}$ & & Revised By / Date & \\
\hline
\end{tabular}

Table 1-1 - Definitions of Cask Drops into South Load Out Pit

\begin{tabular}{|c|c|c|c|c|}
\hline $\begin{array}{c}\text { Case } \\
\text { No. }\end{array}$ & $\begin{array}{c}\text { Drop Weight } \\
\text { (lbs.) }\end{array}$ & $\begin{array}{c}\text { Impact } \\
\text { Velocity (fps) }\end{array}$ & $\begin{array}{c}\text { Impact Energy } \\
\text { (ft.-lbs.) }\end{array}$ & $\begin{array}{c}\text { Analysis } \\
\text { Uncertainty Basis }\end{array}$ \\
\hline 1 & 66,200 & 7.53 & 58,279 & Best Estimate \\
\hline 2 & 66,200 & 28.72 & 848,314 & Best Estimate \\
\hline 3 & 66,200 & 28.72 & 848,314 & Conservative \\
\hline 4 & 66,200 & 44.16 & $2,006,135$ & Best Estimate \\
\hline 5 & 66,200 & 44.16 & $2,006,135$ & Conservative \\
\hline 6 & 66,200 & 7.53 & 58,279 & Conservative \\
\hline 7 & 46,600 & 38.89 & $1,095,554$ & Best Estimate \\
\hline 8 & 46,600 & 38.89 & $1,095,554$ & Conservative \\
\hline 9 & 66,200 & 10.62 & 115,990 & Best Estimate \\
\hline 10 & 66,200 & 10.62 & 115,990 & Conservative \\
\hline 11 & 45,300 & 27.36 & 527,160 & Best Estimate \\
\hline 12 & 45,300 & 27.36 & 527,160 & Conservative \\
\hline
\end{tabular}


SNF-5470, Revision 0

\begin{tabular}{|c|c|c|c|}
\hline $\mathbf{M} \boldsymbol{g} \mathbf{D}^{\text {Professional }}$ & \multicolumn{2}{|c|}{ CALCULATION SHEET } & $\begin{array}{cc}\text { Page No. } & \text { of } \\
6 & 21 \\
\text { CalculationNo. }\end{array}$ \\
\hline \multirow{2}{*}{\multicolumn{2}{|c|}{$\begin{array}{l}\text { Chent/Location } \\
\text { ARES Corporation }\end{array}$}} & Prepared By & M\&D 99-013 \\
\hline & & B. Winkel & Revision No. \\
\hline \multicolumn{2}{|c|}{$\begin{array}{l}\text { Subject } \\
\text { Floor/Wall Joint Evaluation for Drop Loading }\end{array}$} & Checked 8y & 0 \\
\hline \multicolumn{2}{|c|}{$\begin{array}{l}\text { Ret. Orawing(s) } \\
\mathrm{H}-1-21072\end{array}$} & Revised by/Date & \\
\hline
\end{tabular}

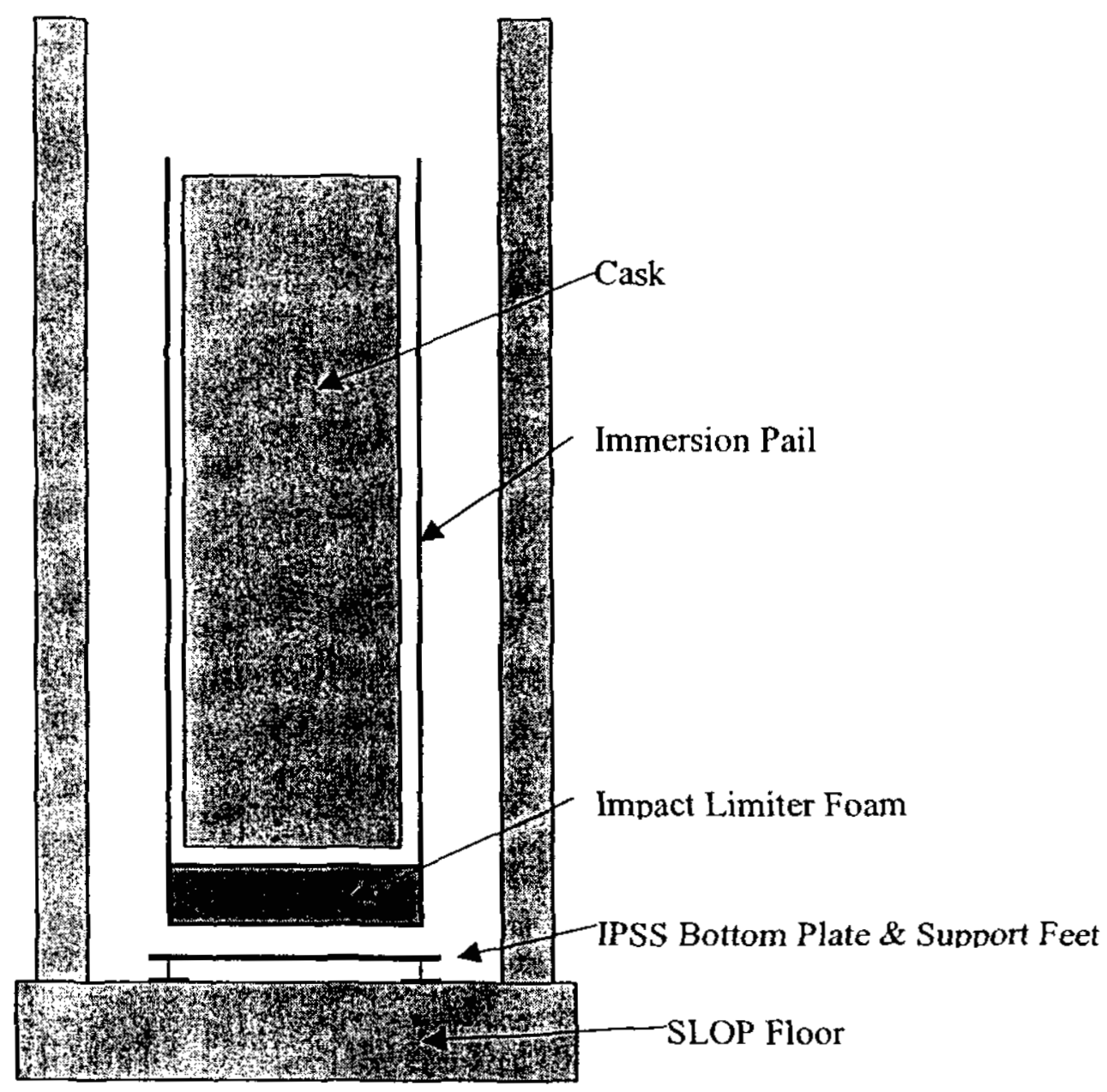

Figure 1-1 - Cask Drop Configuration in South Load Out Pit 


\section{SNF-5470, Revision 0}

\begin{tabular}{|c|c|c|c|}
\hline M\&D ${ }^{\text {Professional }}$ & \multicolumn{2}{|c|}{ CALCULATION SHEET } & \multirow{2}{*}{$\begin{array}{|cc|}\text { Page No. } & \text { of } \\
7 & 21 \\
\text { Calculationno. } & \\
\text { M\&D } & 99-013 \\
\end{array}$} \\
\hline \multicolumn{2}{|l|}{$\begin{array}{l}\text { Chent/Location } \\
\text { ARES Corporation }\end{array}$} & Prepared By & \\
\hline \multicolumn{2}{|c|}{$\begin{array}{l}\text { Subject } \\
\text { Floor/Wall Joint Evaluation for Drop Loading }\end{array}$} & $\begin{array}{l}\text { B. Winkel } \\
\text { Checked By }\end{array}$ & \multirow{2}{*}{$\begin{array}{l}\text { Revision Nó. } \\
\text { Supporting Document No }\end{array}$} \\
\hline \multicolumn{2}{|c|}{$\begin{array}{l}\text { Ref. Drawing(s) } \\
\mathrm{H}-1-21072\end{array}$} & Revised By / Date & \\
\hline
\end{tabular}

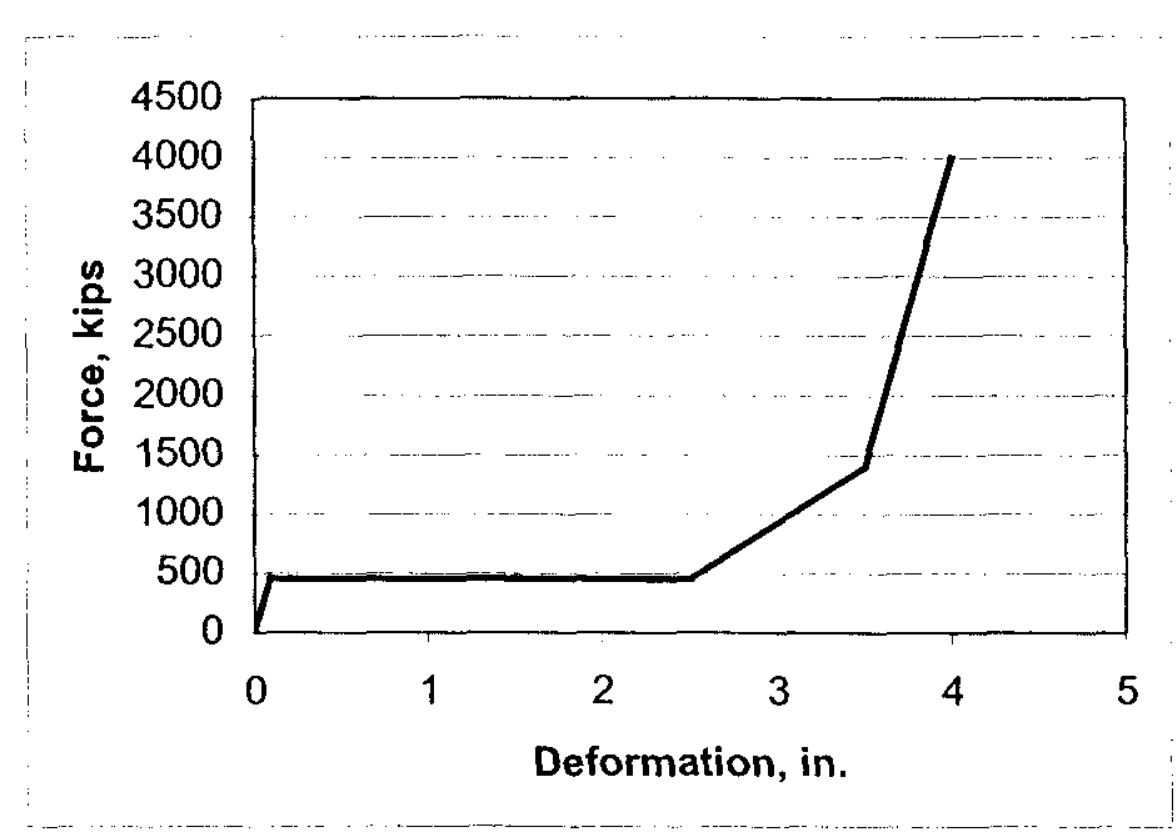

Figure 1-2 - Impact Limiter Assumed Force/Deflection Properties

\subsection{SUMMARY AND CONCLUSIONS}

Using a finite element model of the lower portion of the South Load Out Pit (SLOP), a transient dynamic analysis was performed for each of the cask drop cases listed in Table 1-1. From the dynamic analysis results, residual gap predictions for the wall/floor joint were obtained for each drop case. Immediately following the time of impact, the maximum gap opening occurs, followed by some gap closure due to foundation soil rebound. The remaining "residual gap" is the item of interest for predicting leakage flow.

For the lower energy drops (Energy $<120,000 \mathrm{ft}-\mathrm{lbs}$ ), inelastic stretching of the joint rebar was limited to minor yielding in the middle of the west, south, and north walls of the SLOP. For the remaining drops, significant yielding of the joint rebar occurred in all but the east wall. The east wall is more heavily reinforced and is furthest from the cask drop location. Thus, for the higher energy drops, the wall/floor residual gap is wedge shaped, with the maximum gap on the west wall and essentially zero at the east wall (pivot line). A plot of the maximum residual gap versus drop energy is provided in Figure 2-1. 
SNF-5470, Revision 0

\begin{tabular}{|c|c|c|c|}
\hline M\&D Professional & \multicolumn{2}{|c|}{ CALCULATION SHEET } & \multirow{3}{*}{\begin{tabular}{|cc} 
Page No. & of \\
8 & 21 \\
CalculationNo. \\
M\&D & $99-013$ \\
\end{tabular}} \\
\hline \multirow{2}{*}{\multicolumn{2}{|c|}{$\begin{array}{l}\text { Client/Location } \\
\text { ARES Corporation }\end{array}$}} & Prepared By & \\
\hline & & B. Winkel & \\
\hline \multicolumn{2}{|c|}{$\begin{array}{l}\text { Subject } \\
\text { Floor/Wall Joint Evaluation for Drop Loading }\end{array}$} & Checked By & 0 \\
\hline \multicolumn{2}{|c|}{$\begin{array}{l}\text { Rer. Drawing(s) } \\
\mathrm{H}-1-21072\end{array}$} & Revised By / Date & \\
\hline
\end{tabular}

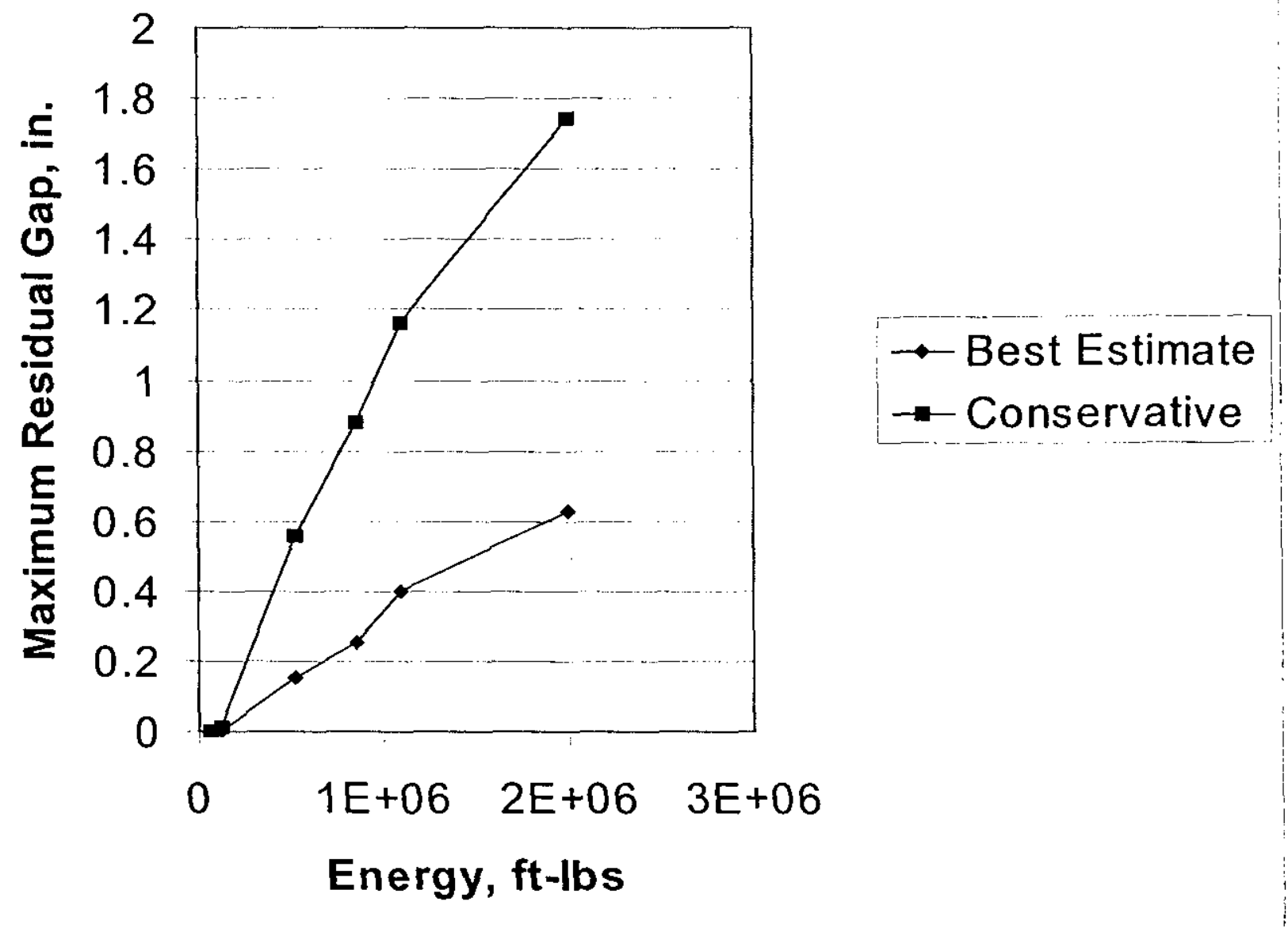

Figure 2-1 - Maximum Residual Gap Versus Impact Energy

\subsection{DESIGN INPUT/ASS UMPTIONS}

\subsection{DESIGN INPUT}

The structural concrete of the K Basin South Load Out Pit is defined in Drawing No. H-1-21072 (Reference 9). As indicated above, the cask handling weights considered for the drop analyses range from 45,300 to $66,200 \mathrm{lbs}$. The floor impact velocities provided as input to this calculation are listed in Table 1-1.

\subsection{LOADS AND LOAD COMBINATIONS}

The potential loads associated with the wall/floor joint are 1) drop loads, 2) wall soil pressure, 3) wall water pressure, 4) dead weight of the concrete structure, 5) water pressure on the floor, and 6) seismic loading. It is conservative to ignore the reinforced concrete structure dead weight loading because the concrete dead weight results in compression of the wall/floor joint, whereas a drop onto the SLOP floor places the joint in 
SNF-5470, Revision 0

\begin{tabular}{|c|c|c|c|}
\hline & \multirow{2}{*}{\multicolumn{2}{|c|}{ CALCULATION SHEET }} & \multirow{3}{*}{\begin{tabular}{|cc} 
Page No. & of \\
9 & 21 \\
Calculation No. \\
M\&D \\
Revision No.
\end{tabular}} \\
\hline $\mathbf{M} \boldsymbol{d} \mathbf{D}{ }^{\text {Professional }}$ & & & \\
\hline \multicolumn{2}{|l|}{$\begin{array}{l}\text { Client/Location } \\
\text { ARES Corporation }\end{array}$} & $\begin{array}{l}\text { Prepared By } \\
\text { R Winkel }\end{array}$ & \\
\hline \multicolumn{2}{|c|}{$\begin{array}{l}\text { Subject } \\
\text { Floor/Wall Joint Evaluation for Drop Loading }\end{array}$} & Checked By & 0 \\
\hline $\begin{array}{l}\text { Ref. Drawing(s) } \\
\mathrm{H}-1-21072 \\
\end{array}$ & & Revised By $/$ Date & \\
\hline
\end{tabular}

tension. The soil and water pressure on the basin walls tend to balance each other. Concerning seismic loading, it is unlikely that a seismic event would coincide with a drop accident and highly unlikely that the peak loadings on the wall/floor joint from a drop and seismic event would coincide in time. Therefore, for the current evaluation only the floor water pressure was considered to be coincident with the drop event.

\subsection{ASSUMPTIONS}

The following assumptions have been applied to the drop evaluation:

1. For analysis simplification, the reinforced concrete structure and supporting soil are modeled as linear components. The effects of concrete cracking on the section stiffness was conservatively ignored (higher concrete stiffness transfers more load to wall/floor joint). The one exception to the concrete structure linear behavior is the use of elastic/plastic rebar elements at the wall/floor joint. Also soil plasticity was considered in estimating the magnitude of floor rebound, as discussed in Section 3.4.

2. It is assumed that the rebar has not experienced significant corrosion. This assumption is primarily based upon the fact that basin inspections have revealed no evidence of rebar corrosion (Reference 3, Table 1). The minimum rebar yield strength of $40 \mathrm{ksi}$ is assumed for the conservative analyses. For the best estimate analyses, average rebar strengths were considered, as well as strain rate effects on the rebar yield strength.

3. A maximum water depth of 21 feet (K West) was used to calculated the maximum pressure loading on the basin floor.

4. The impact is critically damped, i.e. no significant bouncing of the missile occurs following impact. The adequacy of this assumption is addressed in the drop test data benchmark study summarized in Appendix B.

\subsection{MATERIAL PROPE RTIES}

The drop load reaction load path is sensitive to the relative stiffness of the concrete and soil. For example, for a very stiff basin foundation soil, a relatively high portion of the drop load would be reacted directly through the floor to the foundation soil. For a softer foundation soil, more of the drop load is reacted at the wall/floor connection. Therefore, significant attention was given to addressing the uncertainties in the soil stiffness properties, as indicated in the design input parameters discussed below.

The material properties used in the basin drop calculations are listed in Table 3-1 - M. Some explanations are in order to provide the basis for some of the material properties listed. For the maximum concrete elastic modulus value, an upper bound concrete compressive strength of $6000 \mathrm{psi}$ was used $\left(\mathrm{E}=57,000\left[\mathrm{f}_{\mathrm{e}}{ }^{\prime}\right]^{0.5}\right)$ per Section 8.5.1 of Reference 1) which is based upon limited non-destructive testing of the basin concrete (Reference 3) plus other experience with older concrete structures at the Hanford site. The lower bound soil subgrade modulus of $100 \mathrm{psi} /$ in is based upon limited load/deflection testing data found in Reference 10, as discussed in Appendix $U$ of Reference 13. The average subgrade modulus value of $300 \mathrm{psi} / \mathrm{in}$ is based upon typical values listed for dense sand/gravel materials. See, for example, Table 9-1 of Reference 4. 
SNF-5470, Revision 0

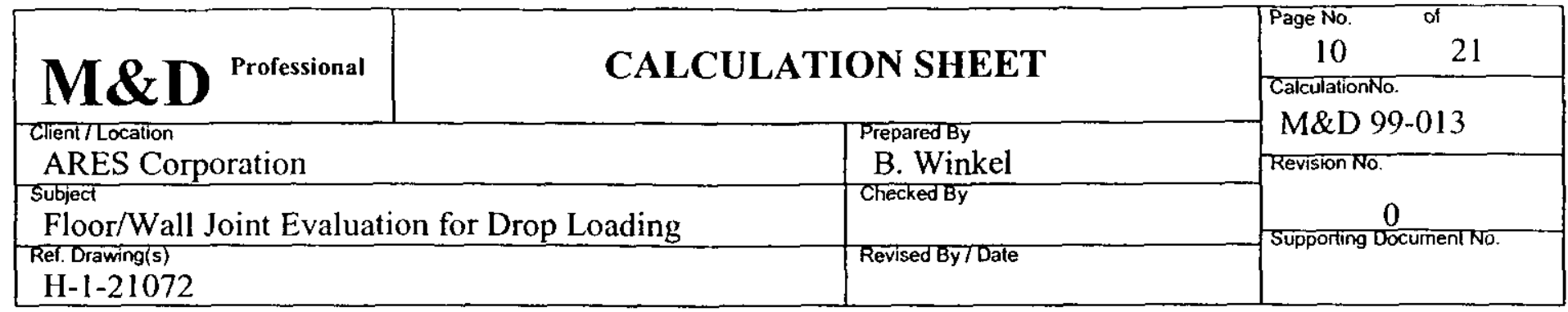

Table 3-1 - Material Properties Used in the Basin Drop Load Calculations

\begin{tabular}{|l|l|l|}
\hline Material & Property & Values Used \\
\hline Concrete & Density & $150 \mathrm{lb} / \mathrm{ft}^{3}$ \\
\hline & Upper Bound Elastic Modulus & $4.4 \times 10^{6} \mathrm{psi}$ \\
\hline & Poisson's Ratio & 0.2 \\
\hline Soil & Compressive Strength & $3000-6000 \mathrm{psi}$ \\
\hline & $\begin{array}{l}\text { Lower Bound Subgrade } \\
\text { Modulus }\end{array}$ & $100 \mathrm{psi} / \mathrm{in}$ \\
\hline Reinforcing Bar & Mverage Subgrade Modulus & $300 \mathrm{psi} / \mathrm{in}$ \\
\hline & Average Yield Strength & $48,000 \mathrm{psi}$ \\
\hline
\end{tabular}

One of the limitations of the elastic soil modeling is the quantifying of the rebound of the floor following the impact loading. The use of elastic springs over predicts the amount of floor rebound. A better estimate of the floor rebound can be obtained by reviewing load test results on foundation piles. The typical pile test involves load magnitudes equal to $200 \%$ of the pile design loads. From Figure $10-11$ of Reference 7 , the rebound results summarized in Table $3-2$ and 
SNF-5470, Revision 0

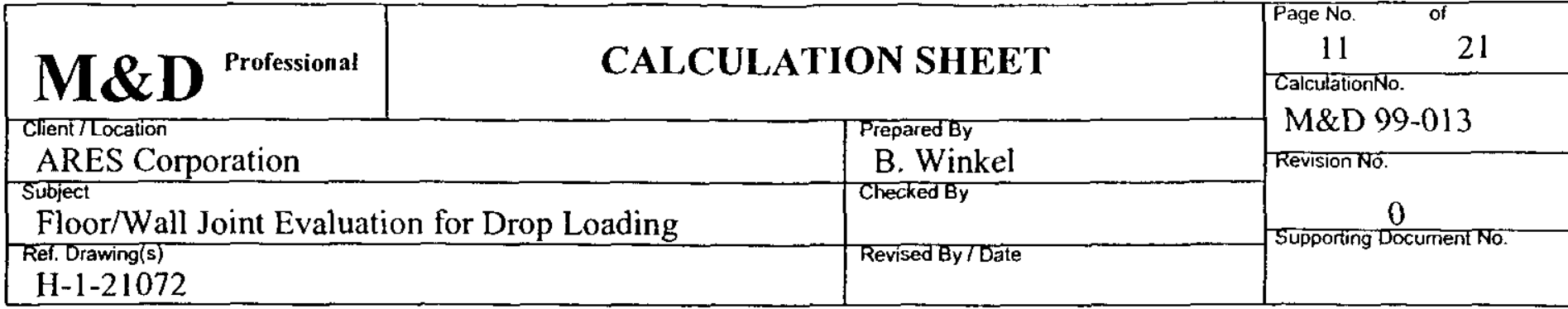

\section{Rebound Versus Max. Soil Deformation}

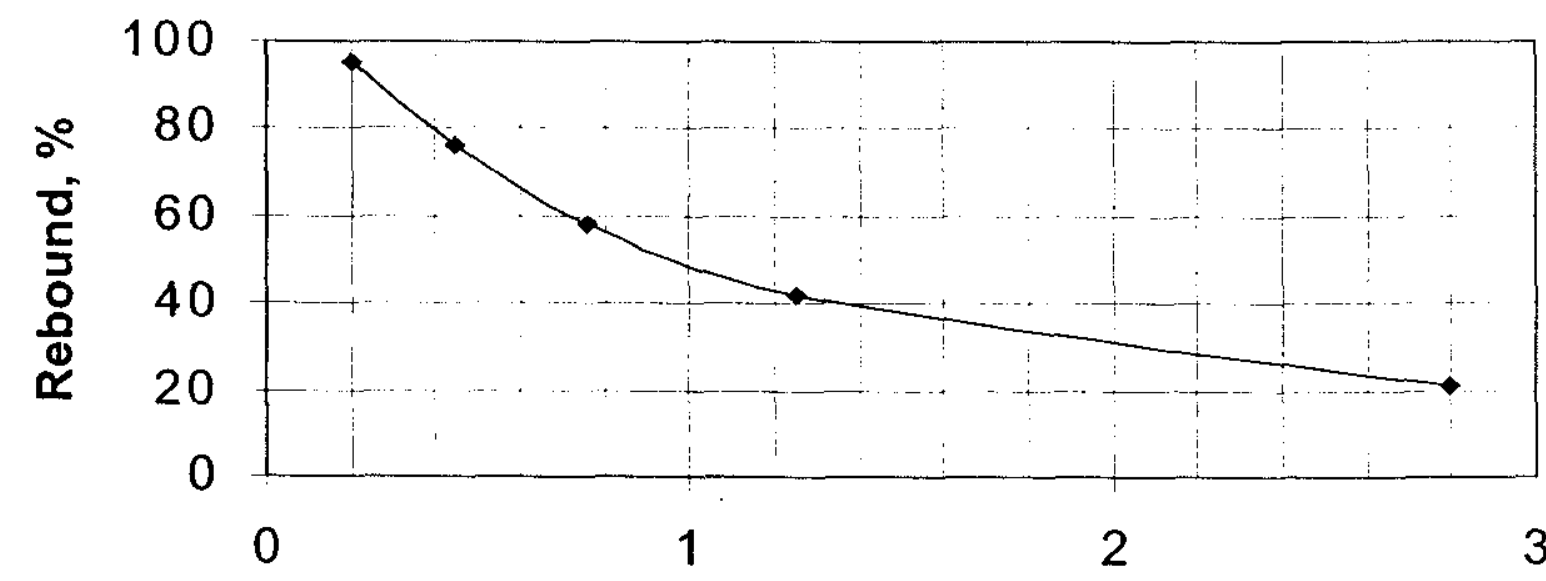

Max. Soil Deformation, in.

Figure 3-1. Note that the pile deformations ranged from 0.20 to $2.80 \mathrm{in}$., and the corresponding rebounds ranged from 95 to $21 \%$.

The Table 3-2/ 
SNF-5470, Revision 0

\begin{tabular}{|c|c|c|c|}
\hline $\mathbf{M} \boldsymbol{E} \mathbf{D}^{\text {Professional }}$ & \multicolumn{2}{|c|}{ CALCULATION SHEET } & $\begin{array}{|cc|}\begin{array}{c}\text { Page No. } \\
12\end{array} & \text { of } \\
\text { Calculation No. } & \\
\end{array}$ \\
\hline \multicolumn{2}{|l|}{ Client/Location } & Prepared By & M\&D 99-013 \\
\hline \multicolumn{2}{|c|}{$\begin{array}{l}\text { Subject } \\
\text { Floor/Wall Joint Evaluation for Drop Loading }\end{array}$} & $\begin{array}{l}\text { B. Winkel } \\
\text { Checked By }\end{array}$ & Revision No. \\
\hline \multicolumn{2}{|c|}{$\begin{array}{l}\text { Ret. Orawing(s) } \\
\mathrm{H}-1-21072\end{array}$} & Revised By/Date & \\
\hline
\end{tabular}

\section{Rebound Versus Max. Soil Deformation}

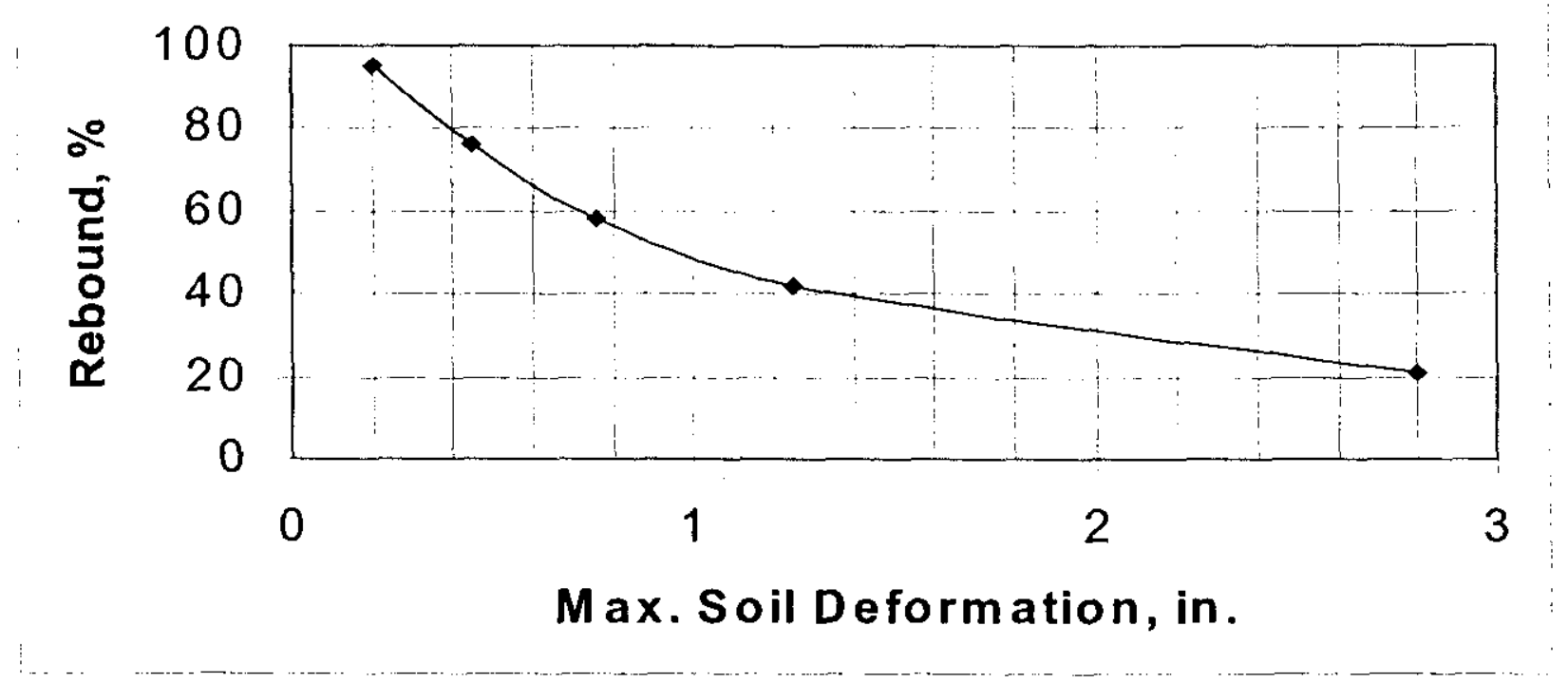

Figure 3-1 rebound percentages were used to approximate the rebound magnitudes for the cask drop accidents. Specifically, the SLOP floor rebound was estimated by correlating the maximum floor deformation to the corresponding gross settlement and rebound percentage from the pile test results. This is conservative in the sense that maximum floor deformations were used, which is about twice the average floor deformation. The approach is unconservative in the sense that the predicted gross floor deformations are based upon an elastic soil model. As shown below, the range of pile settlements are similar to the maximum predicted floor deformations. Also note that for small deformations $(<0.1$ in.), the response is elastic, i.e. the rebound is close to $100 \%$.

The $40 \mathrm{ksi}$ minimum yield strength of the reinforcing bars corresponds to the specified minimum value for the Grade 40 rebar used in the basin construction. The average value is $20 \%$ higher than the minimum, which is typical for rebar fabricated from low carbon steel. See, for example, Figure 3-25 of Reference 13. The yield strengths listed in Table 3-1 are quasi-static values. For drop accidents, the strain rate dependency of the rebar increases the yield strength. For Grade 40 steel, Appendix C of Reference 1, a $20 \%$ increase in the yield strength is specified for strain rates associated with impact loading. This $20 \%$ strain rate increase was applied to both the best estimate and conservative dynamic analyses. 
SNF-5470, Revision 0

\begin{tabular}{|c|c|c|c|}
\hline & \multirow{2}{*}{\multicolumn{2}{|c|}{ CALCULATION SHEET }} & \multirow{3}{*}{\begin{tabular}{|lr} 
Page No. & of \\
13 & 21 \\
CalculatioriNo. & \\
M\&D & $99-013$
\end{tabular}} \\
\hline M\&D Professional & & & \\
\hline \multicolumn{2}{|l|}{$\begin{array}{l}\text { Cleent/location } \\
\text { ARES Corporation }\end{array}$} & $\begin{array}{l}\text { Prepared By } \\
\text { B. Winkel }\end{array}$ & \\
\hline \multicolumn{2}{|c|}{$\begin{array}{l}\text { Subject } \\
\text { Floor/Wall Joint Evaluation for Drop Loading }\end{array}$} & Checked By & \multirow{2}{*}{$\begin{array}{l}\text { Revision No. } \\
\qquad \begin{array}{l}0 \\
\text { Supporting Document }\end{array}\end{array}$} \\
\hline \multicolumn{2}{|c|}{$\begin{array}{l}\text { Ref. Drawing(s) } \\
\mathrm{H}-1-21072\end{array}$} & Revised By/Dale & \\
\hline
\end{tabular}

Table 3-2 - Pile Load Tests, Rebound Results

\begin{tabular}{|c|c|c|c|}
\hline Test Load (kips) & Gross Settlement (in.) & Rebound (in.) & $\begin{array}{c}\text { Rebound (\% of Gross } \\
\text { Settlement) }\end{array}$ \\
\hline 100 & 0.20 & 0.19 & 95 \\
\hline 200 & 0.45 & 0.34 & 76 \\
\hline 300 & 0.76 & 0.44 & 58 \\
\hline 400 & 1.25 & 0.53 & 42 \\
\hline 500 & 2.80 & 0.60 & 21 \\
\hline
\end{tabular}

\section{Rebound Versus Max. Soil Deformation}

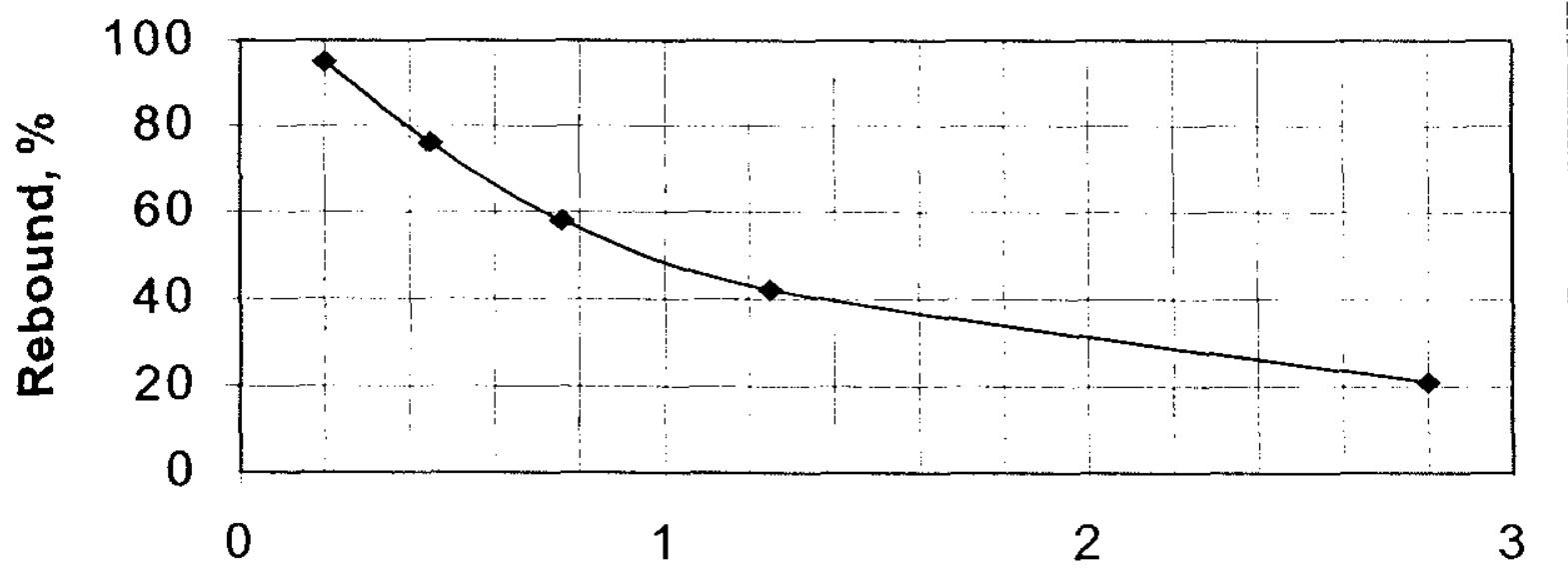

Max. Soil Deformation, in.

Figure 3-1 - Soil Rebound Percentage Versus Maximum Deformation 
SNF-5470, Revision 0

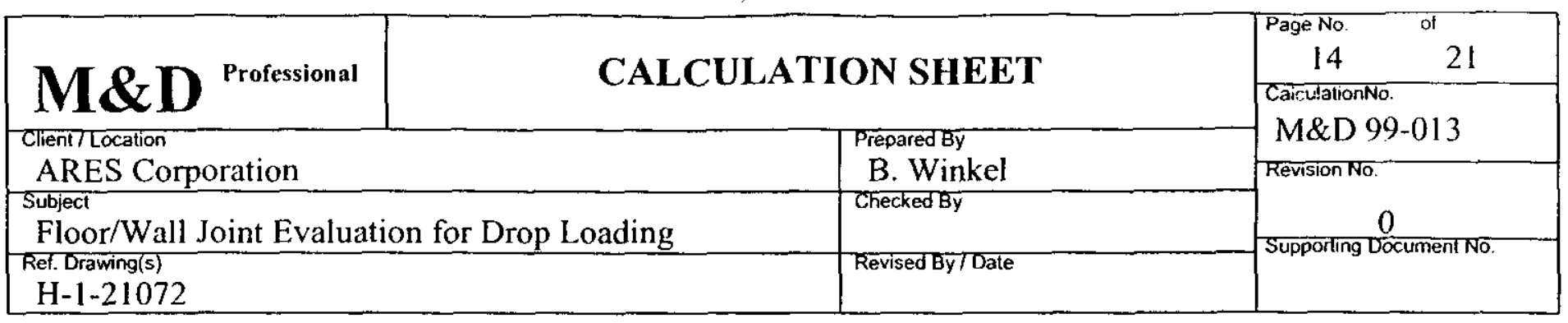

\subsection{ANALYSIS APPROA CH}

Transient dynamic analyses were performed for the twelve cask drop cases listed in Table 1-1, using the ANSYS computer code. A description of the ANSYS finite element model of the South Load Out Pit follows below.

\subsection{FINITE ELEMENT MODEL}

The ANSYS structural model of the K Basin wall/floor joint developed for the dynamic analysis is shown in Figure 4-1. This model was adapted from a model that has been previously used for SLOP drop analyses and has been subjected to checking and independent reviews. Note that to keep the analysis run times to reasonable values, only the lower portion of the SLOP walls was modeled. This model simplification is conservative, but reasonable, based upon prior experience with drop analyses in the SLOP. Vertical support to the floor is provided by soil springs, which are input using the elastic foundation stiffness (EFS) parameter for the ANSYS SHELL63 elements.

The top of the SLOP wall was fixed vertically, which was found to be reasonably conservative in prior experience since the actual walls are constrained by soil friction and attachment to the basin. Since the focus of the wall/floor joint evaluation is yielding in the reinforcing bars, which connect the floor to the wall, the rebar was explicitly modeled using elastic/plastic spar elements (ANSYS LINK8). The basis for the rebar element modeling details is described in the next section.

\subsubsection{Wall/floor Rebar Modeling Details}

Accurate predictions of rebar stress and strain (and joint deformations) require an accurate representation of rebar bond slip at the wall/floor construction joint. The effective length of the joint rebar increases with load magnitude, and can be quantified by using the simplifying assumption that the rebar stress is balanced by a uniform shear stress at the O.D. of the rebar equal to the bond strength. Reasonable estimates of rebar "effective lengths" are 16 inches (\#10 rebar) and 15 inches (\#9 rebar), as derived below.

Based upon the testing at the University of Illinois (Reference 5), a reasonable estimate of the bond strength of deformed bars is

$$
\sigma_{\text {boud }}=14 \sqrt{f_{c}^{\prime}}
$$

where $f_{c}^{\prime}$ is the concrete design strength and $\sigma_{b o n d}$ is the ultimate bond strength.

When the rebar reaches the material yield strength, the length of the distributed ultimate bond stress can be estimated by assuming that the rebar stress is balanced by a uniform shear/bond stress at the rebar periphery, equal to the above bond strength. For the uniform bond stress assumption, the rebar stress would be transferred in a linear fashion from the yield stress at the joint down to zero at the end of the balanced rebar bond strength stress. The associated length of rebar achieving the bond ultimate strength is found by equating the bond force to the rebar yield force: 
SNF-5470, Revision 0

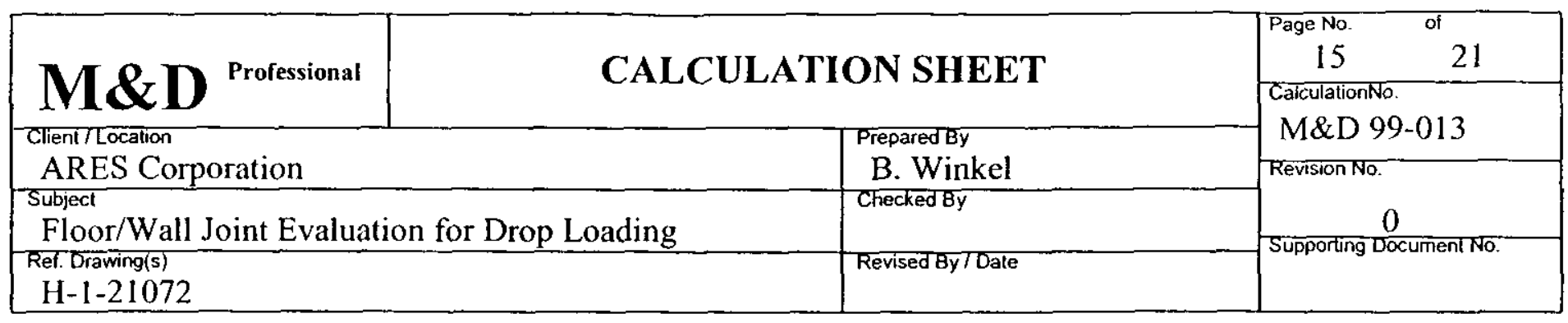

$$
\begin{gathered}
\sigma_{y} A_{\text {rebar }}=\sigma_{\text {bond }}\left(\text { circum }_{\text {rebar }}\right) l_{\text {eff }} \\
\sigma_{y} \frac{\pi d^{2}}{4}=14 \sqrt{f_{c}^{\prime}}(\pi d) l_{e f f} .
\end{gathered}
$$

Solving for $l_{e f f}$ :

$$
l_{\text {eff }}=\frac{\sigma_{y} d}{4\left(14 \sqrt{f_{c}^{\prime}}\right)}=\frac{40,000(0.625)}{56 \sqrt{3,000}}=8.15 \mathrm{in} .
$$

where $\sigma_{y}$ is the rebar yield strength, $d$ is the rebar diameter ( $\# 5$ bars), and $f_{c}{ }^{\prime}$ is the concrete 28 -day design strength. Repeating the same calculation for a $d$ value of 0.875 inches ( $\# 7$ bars) results in an effective length of 11.4 inches. For modeling simplicity and conservatism, the shorter length of $8.15 \mathrm{in}$. was used for both bar sizes.

The actual effective rebar length varies with load magnitude, due to a progressive bond slip, which is highest near the construction joint. The higher bond slip near the joint results in a bond stress of near zero, adjacent to the joint, which means that upon yielding, the rebar stress does not drop below the yield stress for some finite distance from the joint. Based upon comparisons with rebar bond test load/deflection data, it is reasonable to assume an effective length of half of $l_{\text {eff }}$ on each side of the joint, for a total effective length of $l_{\text {eff. }}$. The adequacy of this approximation in selecting an effective rebar modeling length at the construction joint was bench-marked by comparing the rebar model, with a constant effective length of $l_{\text {eff }}$ deformation predictions with actual rebar bond test data, as shown in Figure 4-2. The "model" prediction shown was based upon the same geometry and material properties used in the test program (\#7 rebar, $\mathrm{f}_{\mathrm{y}}=42 \mathrm{ksi}, \mathrm{f}_{\mathrm{c}}{ }^{\prime}=$ $5000 \mathrm{psi}$ ). Note that the bilinear shape of the constant length model response curve differs somewhat from the rebar test data curves, but is close to the average response at a stress level of $100 \%$ of the yield strength.. Thus, the effective length, $l_{e f f}$, derived above, was judged to be adequate for the elastic/plastic rebar elements in the finite element model shown in Figure 4-1. 
SNF-5470, Revision 0

\begin{tabular}{|c|c|c|c|}
\hline \multirow[b]{2}{*}{ M\& } & \multirow{2}{*}{\multicolumn{2}{|c|}{ CALCULATION SHEET }} & \multirow{3}{*}{$\begin{array}{|cc|}\begin{array}{c}\text { Page No. } \\
16\end{array} & \text { of } \\
\text { CalculationNo. } \\
\text { M\&D }\end{array}$} \\
\hline & & & \\
\hline \multirow{2}{*}{\multicolumn{2}{|c|}{$\begin{array}{l}\text { Client } / \text { Location } \\
\text { ARES Corporation }\end{array}$}} & Prepared By & \\
\hline & & B. Winkel & Revision No. \\
\hline \multicolumn{2}{|c|}{$\begin{array}{l}\text { Subject } \\
\text { Floor/Wall Joint Evaluation for Drop Loading }\end{array}$} & Checked By & 0 \\
\hline \multicolumn{2}{|c|}{$\begin{array}{l}\text { Ret. Drawing(s) } \\
\mathrm{H}-1-21072\end{array}$} & Revised By/Date & \\
\hline
\end{tabular}

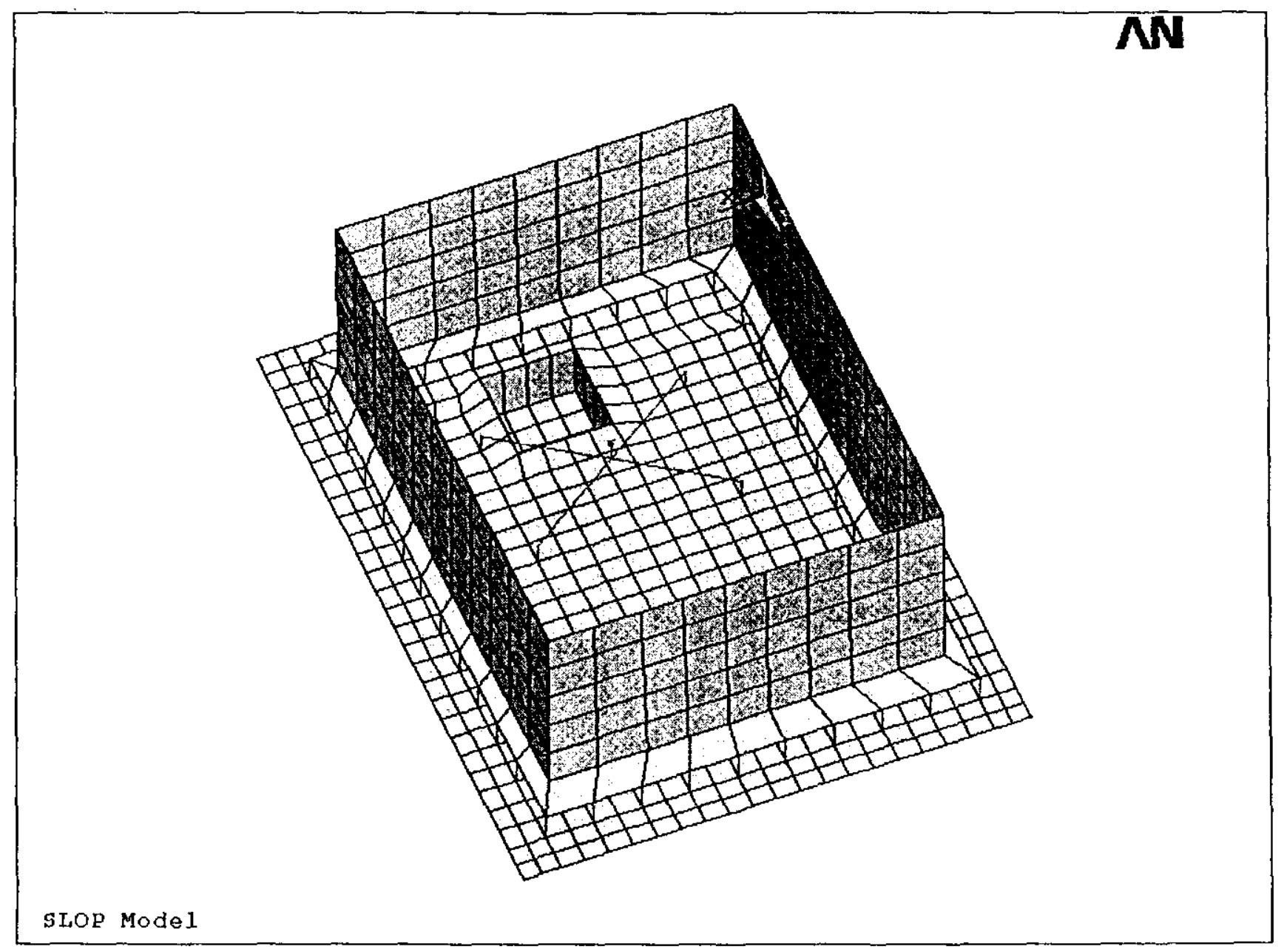

Figure 4-1 - Finite Element Model of the K Basin Wall/Floor Joint 
SNF-5470, Revision 0

\begin{tabular}{|c|c|c|c|}
\hline & \multirow{2}{*}{\multicolumn{2}{|c|}{ CALCULATION SHEET }} & \multirow{3}{*}{ 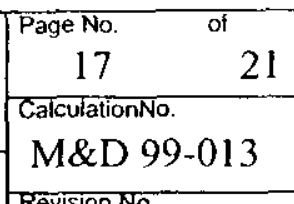 } \\
\hline Mien Professional & & & \\
\hline $\begin{array}{l}\text { Clent/location } \\
\text { ARES Corporation }\end{array}$ & & $\begin{array}{l}\text { PrepäredBy } \\
\text { B. Winkel }\end{array}$ & \\
\hline $\begin{array}{l}\text { Subject } \\
\text { Floor/Wall Joint Evalua }\end{array}$ & oading & & 0 \\
\hline $\begin{array}{l}\text { Rer. Drawing(s) } \\
\text { H-1-21072 }\end{array}$ & & Revised By/Date & \\
\hline
\end{tabular}

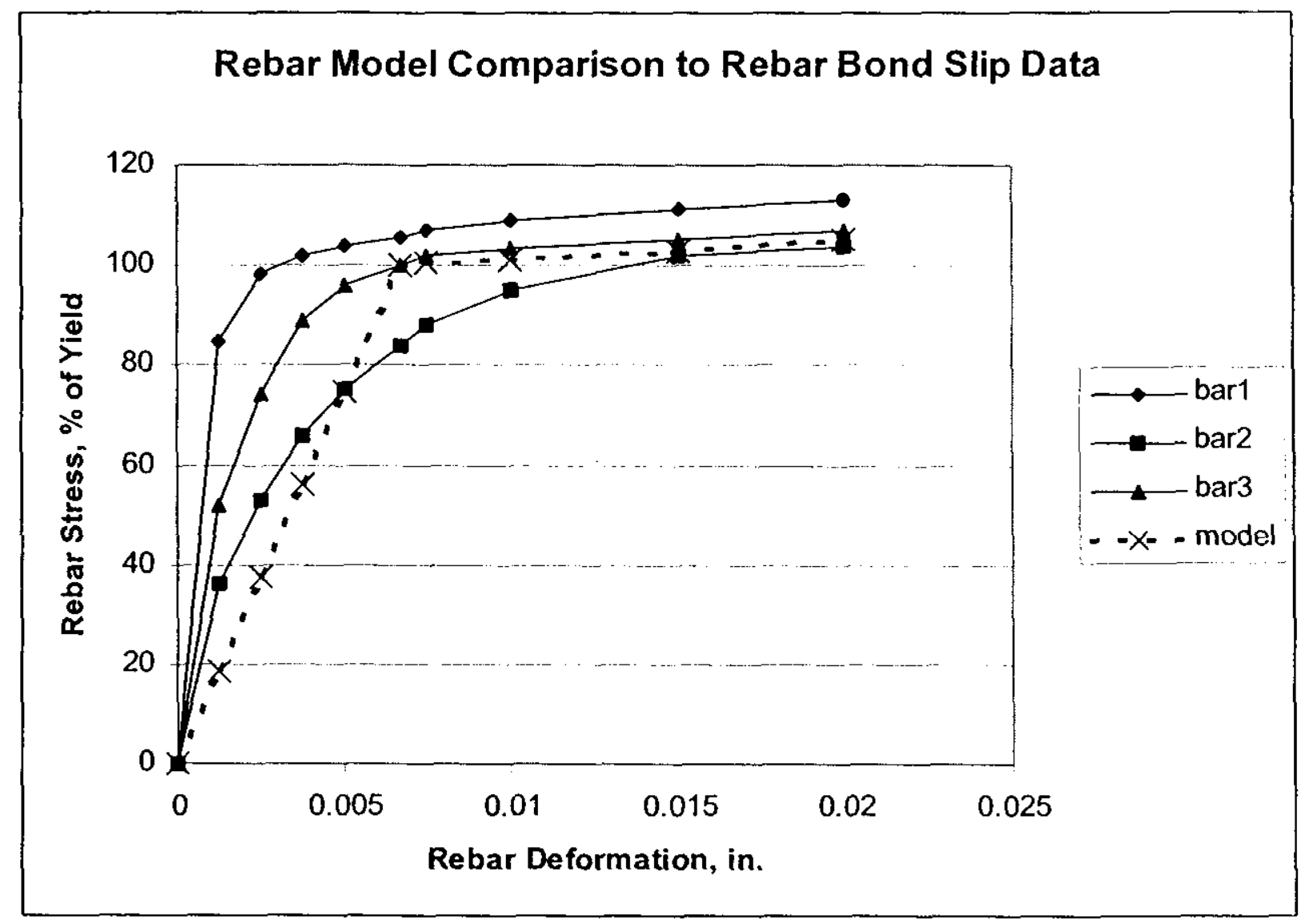

Figure 4-2 -- Evaluation of Construction Joint Rebar Model Adequacy 
SNF-5470, Revision 0

\begin{tabular}{|c|c|c|c|}
\hline M\&D $\mathbf{D}^{\text {Professional }}$ & \multicolumn{2}{|c|}{ CALCULATION SHEET } & \begin{tabular}{|c|c|} 
Page No. & of \\
18 & 21 \\
CalculationNo.
\end{tabular} \\
\hline \multicolumn{2}{|l|}{$\begin{array}{l}\text { Client } / \text { Location } \\
\text { ARES Corporation }\end{array}$} & $\begin{array}{l}\text { Prepared By } \\
\text { B Winkel }\end{array}$ & M\&D 99-013 \\
\hline \multicolumn{2}{|c|}{$\begin{array}{l}\text { Subject } \\
\text { Floor/Wall Joint Evaluation for Drop Loading }\end{array}$} & $\begin{array}{l}\text { B. Winket } \\
\text { Checked By }\end{array}$ & 0 \\
\hline \multicolumn{2}{|c|}{$\begin{array}{l}\text { Ret. Drawing(s) } \\
\text { H-1-21072 }\end{array}$} & Revised By / Date & \\
\hline
\end{tabular}

\subsubsection{Loading and Other A nalysis Details}

\section{Drop Loading}

The drop loading was applied by using a mass element (ANSYS MASS21), with a mass magnitude equal to the cask mass value listed in Table 1-1. The lateral position of the mass element corresponded to the centerline of the cask. Contact of the mass element to the floor was made using rigid links to the elements representing the four Immersion Pail Support Structure (IPSS) feet which are appropriately positioned on the floor. Gap and damper elements (ANSYS CONTAC52 and COMBIN14) connect the ends of the rigid links to the floor, as shown in Figure 4-1. The mass was given an initial velocity corresponding to the values listed in Table 1-1.

\section{Missile/Floor Contact Element Properties}

A total "contact stiffness" of $1 \times 10^{6} \mathrm{lb} /$ in was used for the gap elements representing the IPSS feet, based upon the benchmark evaluation presented in Appendix B. Damper elements (ANSYS COMBIN14) were used in parallel with the impact spring elements. To achieve critical damping (see Assumption 4, Section 3.3), the following damper element constant was used for the 66,200-lb. basket (Reference 14, page 23):

$$
c_{c}=2 \sqrt{\mathrm{km}}=2 \sqrt{1 \times 10^{6} \cdot 66,200 / 386.4}=26,000 \mathrm{lb} / \mathrm{in} / \mathrm{sec} .
$$

The corresponding values for the $46,600-\mathrm{lb}$. and $45,300-\mathrm{lb}$. casks are 22,000 and $21,700 \mathrm{lb} / \mathrm{in} / \mathrm{sec}$, respectively.

\section{Floor Pressure Loading}

Using the SLOP water depth of 22.5 feet (K East basin), the water pressure on the floor was calculated to be

$$
p_{\text {water }}=\gamma h=62.4(22.5)=1404 p s f=9.75 p s i .
$$

\section{Transient Time Step}

Transient dynamic analyses were performed (ANSYS ANTYPE,TRANS) using automatic time stepping with an initial time step of $0.5 \times 10^{-4}$ seconds. Drop analyses were performed for all cask drop cases listed in Table 1-1. 
SNF-5470, Revision 0

\begin{tabular}{|c|c|c|c|}
\hline M\&D ${ }^{\text {Professional }}$ & \multicolumn{2}{|c|}{ CALCULATION SHEET } & \multirow{4}{*}{\begin{tabular}{|c}
19 \\
CalculationNo. \\
M\&D 99-013 \\
Revision No. \\
0 \\
Supporting Document No
\end{tabular}} \\
\hline $\begin{array}{l}\text { Chent/Location } \\
\text { ARES Corporation }\end{array}$ & & 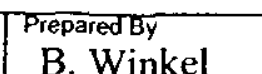 & \\
\hline $\begin{array}{l}\text { Subject } \\
\text { Floor/Wall Joint Evalua }\end{array}$ & oading & & \\
\hline $\begin{array}{l}\text { Ref. Orawing(s) } \\
\mathrm{H}-1-21072\end{array}$ & & Revised By/Date & \\
\hline
\end{tabular}

\subsection{ANALYSIS RESULTS}

The typical SLOP floor deformation pattern for a cask drop event is shown in Figure 5-1. Note that the maximum deformation occurs on the west side of the floor and is close to zero on the east end. This pattern is due to 1) the eccentricity of the load (closer to the west side), and 2) larger rebar on the east side of the wall/floor joint. Using the outside rebar on the middle of the west wall as the "maximum gap". As described in Section 4.1.2, this maximum gap occurring during the transient is reduced by an approximate rebound value, resulting in the final maximum "residual gap" values. It is also noted that the wedged-shape joint gap described above is not typical for the low energy drops (Case Nos. 1, 6, 9, and 10). For the low energy drops, the rebar at the wall intersections (corners) does not yield resulting in a floor deformation pattern which is somewhat bowl-shaped.

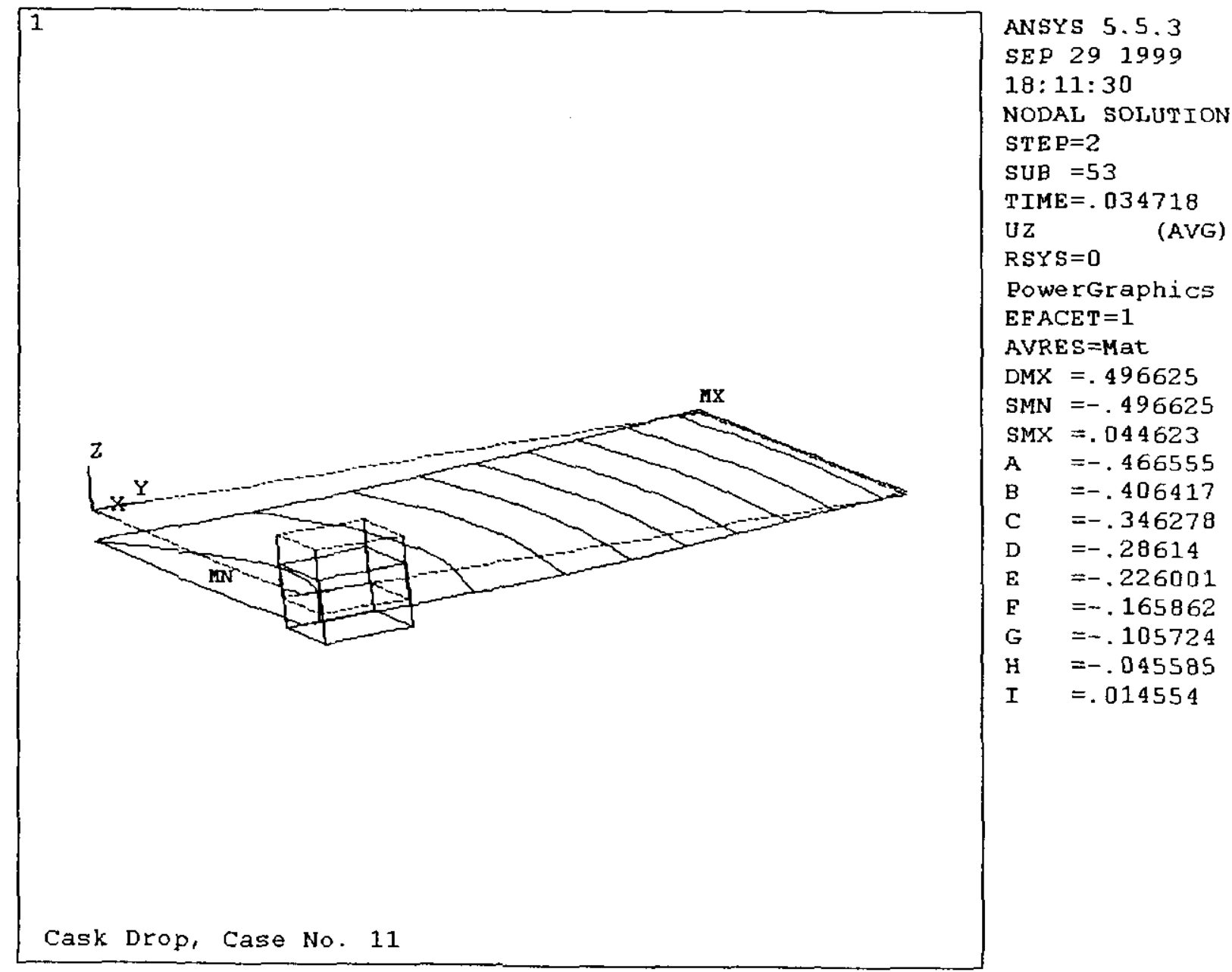

\section{Figure 5-1 - Typical Floor Deformation Pattern}

The residual gap predictions for the twelve drop cases are listed in Table 5-1. 
SNF-5470, Revision 0

Floor/Wall Joint Evaluation for Drop Loading Rer. Drawing(s)

H-1-21072

Table 5-1 - Residual Gap Predictions for the Cask Drop Cases

\begin{tabular}{|c|c|c|c|}
\hline Drop Case No. & $\begin{array}{c}\text { Impact Energy } \\
\text { (ft-lbs) }\end{array}$ & $\begin{array}{c}\text { Analysis } \\
\text { Category }\end{array}$ & $\begin{array}{c}\text { Maximum } \\
\text { Residual Gap (in.) }\end{array}$ \\
\hline 1 & 58,279 & Best Estimate & $<0.001$ \\
\hline 2 & 848,314 & Best Estimate & 0.25 \\
\hline 3 & 848,314 & Conservative & 0.88 \\
\hline 4 & $2,006,135$ & Best Estimate & 0.63 \\
\hline 5 & $2,006,135$ & Conservative & 1.74 \\
\hline 6 & 58,279 & Conservative & $<0.001$ \\
\hline 7 & $1,059,554$ & Best Estimate & 0.40 \\
\hline 8 & $1,059,554$ & Conservative & 1.16 \\
\hline 9 & 115,990 & Best Estimate & $<0.001$ \\
\hline 10 & 115,990 & Conservative & $<0.010$ \\
\hline 11 & 527,160 & Best Estimate & 0.15 \\
\hline 12 & 527,160 & Conservative & 0.56 \\
\hline
\end{tabular}

A graphical presentation of the residual gap predictions is provided in Figure 2-1. 
SNF-5470, Revision 0

\begin{tabular}{|c|c|c|c|}
\hline M\&D $\mathbf{D}^{\text {Professional }}$ & \multicolumn{2}{|c|}{ CALCULATION SHEET } & \multirow{2}{*}{$\begin{array}{|cc|}\text { Page No. } & \text { of } \\
21 & 21 \\
\text { CalculationNo. } \\
\text { M\&D 99-013 } \\
\text { Revision No. }\end{array}$} \\
\hline $\begin{array}{l}\text { Client } / \text { Location } \\
\text { ARES Corporation }\end{array}$ & & $\begin{array}{l}\text { Prepared By } \\
\text { B. Winkel }\end{array}$ & \\
\hline $\begin{array}{l}\text { Subject } \\
\text { Floor/Wall Joint Evalua }\end{array}$ & oading & Checked By & 0 \\
\hline $\begin{array}{l}\text { Ref. Drawing(s) } \\
\text { H-1-21072 }\end{array}$ & & Revised By/Date & \\
\hline
\end{tabular}

\subsection{REFERENCES}

1. ACI 1995, Code Requirements for Nuclear Safety Related Concrete Structures, ACI 349, American Concrete Institute, Detroit, Michigan.

2. ACI 1995a, Building Code Requirements for Reinforced Concrete, ACI-318, American Concrete Institute, Detroit, Michigan.

3. ICF Kaiser 1996, JCS IK-95-01419, 105 KW Basin Structural Inspection, Correspondence No. SCS-W96-939, ICF Kaiser Hanford Company, Richland, Washington.

4. Bowles 1988, Foundation Analysis and Design, McGraw-Hill, New York City, New York.

5. Lai, Shing Sham, G. T. Will and S. Otani, 1984, "Model for Inelastic Biaxial Bending of Concrete Members", ASCE Journal of Structural Engineering, V. 110, No. 11.

6. Collier, S. T., 1947, Bond Characteristics of Commercial and Prepared Reinforcing Bars, Journal of the American Concrete Institute, V. 43, Detroit Michigan.

7. Liu, C. and Evett, J. B., 1992, Soils and Foundations, $3^{\text {rd }}$ Edition, Prentice Hall, Englewood Cliffs, New Jersey.

8. WHC-SD-WM-SAR-062, K Basins Safety Analysis Report, Revision 3J, dated December 8, 1998.

9. Drawing H-1-21072, Structural Concrete Sections and Details at Elev. 0'-0" Storage and Transfer Area, Sheet 1, Revision 5, dated April 1, 1983.

10. Udine, G, 1956, HAPO Soil Information, General Electric Company, Richland, Washington.

11. Teng, W. C., 1962, Foundation Design, Prentice-Hall, Englewood Cliffs, New Jersey.

12. Shannon and Wilson, 1999, "Geotechnical Engineering Review of K Basins Soil Properties; $100 \mathrm{~K}$ Area, Hanford Site, Washington", Shannon and Wilson, Inc., Richland, Washington.

13. DESH 1999, Conceptual Design Report - Cask Loadout System/Cask Drop Redesign for the Immersion Pail Support Structure and Operator Interface Platform, $105 \mathrm{~K}$ West Basin, Project A.5/A.6, HNF-4576, Rev. 0, Duke Engineering Services, Richland, Washington. Macgregor, James G., 1992, Reinforced Concrete Mechanics and Design, $2^{\text {nd }}$ Edition, Prentice Hall, Englewood Cliffs, New Jersey.

14. Thomson 1972, Theory of Vibration with Applications, Prentice-Hall, Inc. New Jersey.

15. LLNL, 1998, Summary and Evaluation of Low-Velocity Impact Tests of Solid Billet Onto Concrete Pads, NUREG/CR-6608, Lawrence Livermore National Laboratory, Livermore, California. 
SNF-5470, Revision 0

\begin{tabular}{|c|c|c|c|}
\hline M\&D ${ }^{\text {Professional }}$ & \multicolumn{2}{|c|}{ CALCULATION SHEET } & \multirow{2}{*}{\begin{tabular}{|cc} 
Page No. & of \\
A1 & A6 \\
CalculationNo. & \\
M\&D & $99-013$
\end{tabular}} \\
\hline $\begin{array}{l}\text { Client/Location } \\
\text { ARES Corporation }\end{array}$ & & Prepared By & \\
\hline $\begin{array}{l}\text { Subject } \\
\text { Floor/Wall Joint Evaluat }\end{array}$ & oading & $\begin{array}{l}\text { B. Winkel } \\
\text { Checked By }\end{array}$ & $\begin{array}{r}\text { Revision No. } \\
0\end{array}$ \\
\hline $\begin{array}{l}\text { Ref. Drawing(s) } \\
\mathrm{H}-\mathrm{I}-21072\end{array}$ & & Revised By $/$ Date & Supporting Document No. \\
\hline
\end{tabular}

APPENDIX A

TYPICAL ANSYS INPUT FILE 
SNF-5470, Revision 0

\begin{tabular}{|c|c|c|c|}
\hline & \multirow{2}{*}{\multicolumn{2}{|c|}{ CALCULATION SHEET }} & \multirow{3}{*}{\begin{tabular}{|c|} 
Page No. \\
A2
\end{tabular}} \\
\hline M\&D $\mathcal{D}^{\text {Professional }}$ & & & \\
\hline \multicolumn{2}{|l|}{$\begin{array}{l}\text { Client / Location } \\
\text { ARES Corporation }\end{array}$} & Prepared By & \\
\hline \multirow{2}{*}{\multicolumn{2}{|c|}{ Joint Evaluation for Drop Loading }} & D. Wecked By & \\
\hline & & & 0 \\
\hline \multicolumn{2}{|l|}{$\begin{array}{l}\text { Ref. Drawing(s) } \\
\text { H-1-21072 }\end{array}$} & Revised By/Date & \\
\hline
\end{tabular}

/prep7

/title, Load Out Pit Model

!element types

et, 1,63 !concrete

et, 2,8 !rebar

et,3,52 !gap elements (outside rebar)

et, 4,4 !rigid link

et, $5,39,1,3$ !nonlinear spring(foam)

et, $6,21,, 2$ !impacting mass element

et, $7,14,3$ !dashpot

!real constants

$\mathrm{r}, 1,17.75,,, 100$ !West Pit Wall

$r, 2,16,,, 0 \quad$ !Remaining Pit Walls

$r, 3,27,,, 100 \quad$ !Basin Walls

$\mathrm{r}, 4,21,,, 100$ !Pit Floor

$r, 5,24,,, 100$ !Basin Floor/Pit E. Wall (bottom)

$\mathrm{r}, 6,18,,,, 100 \quad$ !Sump Bottom Thickness

$\mathrm{r}, 7,0.31 \quad$ ! $\$ 5$ rebar

$\mathrm{r}, 8,0.60 \quad$ ! \#7 rebar

$\mathrm{r}, 9,17 \quad$ !rigid plate, floor/wall conn.

$\mathrm{r}, 10,1 \mathrm{e} 9 \quad$ !gap element spring

$\mathrm{r}, 11,1 \mathrm{e} 8,, 0.0,17965$ !pit walls friction

$\mathrm{r}, 12,1 \mathrm{e} 8,,, 11700$ !basin/channel walls friction

$r, 13,171.3 \quad$ !impacting mass $(64,900 \mathrm{lbs}$.)

$r, 14,2.5 \mathrm{e} 5,3446$ !impact gap element

$r, 15,0,6545$ !impact dashpot element constant

$r, 16,10,1000,1000,10,10,, 1000$ !rigid link

$r, 17,0,0,085,450000,2.5,450000$

rmore,3.5,1.4e6,4,4e6 !nonlinear spring (39)

!material prop.

!concrete

mp,ex, $1,4.4 \mathrm{e} 6$

mp, nuxy, $1,0.2$

mp,dens, $1, .000225$

!rebar, elas./plas.

mp,ex, $2,29 \mathrm{e} 8$

mp,nuxy, $2,0.3$

Tb,Bkin, 2,1

Tbdata, $1,48 \mathrm{e} 3,8 \mathrm{e} 5$

!rigid plates, wall-to-floor conn.

mp,ex,3,4.0e6

mp,nuxy, $3,0.2$

!inelas. floor

mp, ex, 4,4.4e6

mp,nuxy, $4,0.2$

mp,dens, $4, .000225$

Tb,Bkin, 4,1

Tbdata, $1,457,8 \mathrm{e} 5$ !pseudo yield strength, floor eles.

$\mathrm{n}, 1,0,0,0$

$\mathrm{n}, 3,9.32$

fill $\mathrm{n}, 21,118.68$

fill, 3,21

n, 23,128

fill, 21,23

ngen, $3,23,1,23, \ldots, 5.16$

ngen, $3,23,47,69, \ldots 5.685$

ngen, $2,23,93,115,, 5.83$

ngen, $2,23,116,118,, 5.83$

$\mathrm{n}, 143,18.94,33.35$

fill, 141,143

n, $157,109.06,33.35$

fill, 143,157

$\mathrm{n}, 159,118.68,33.35$

fill, 157,159

$\mathrm{n}, 161,128,33.35$

fill, 159,161

ngen, $17,23,139,161,, 5.831$

ngen, $2,414,116,138, \ldots 104.96$

ngen, $2,460,93,115,,, 116.62$

ngen, $4,23,553,575,, 6.083$

ngen, $3,23,622,644,, 4.72$

!modify nodes @ sump boundary

n, $126,58,24.75$

$\mathrm{n}, 130,82,24.75$

fill

n, $218,58,48.75$

$\mathrm{n}, 222,82,48.75$

fill

fill, 126,218,3

fill, $130,222,3$

!n, 103,57.93,20.7

!n, 107,82.2,20.7

!fill

$\mathrm{n}, 241,57.6,56.2$

$\mathrm{n}, 245,83.4,56.2$

fill

!generate floor elements

real, 4

type, 1

mat, 1

$\mathrm{e}, 1,2,25,24$

egen, $22,1,-1$

egen, $29,23,-23$

!delete eles. in sump hole

edele, 121,124

edele, 143,146

edele, 165,168

edele, 187,190

!generate sump elements

real, 6

ngen, $2,700,126,130,,,-16.5$

ngen. $2,700,218,222,, \ldots,-16.5$ 
SNF-5470, Revision 0

\begin{tabular}{|c|c|c|c|}
\hline & \multirow{2}{*}{\multicolumn{2}{|c|}{ CALCULATION SHEET }} & \multirow{3}{*}{\begin{tabular}{|l|} 
Page No. \\
A3 \\
CaculationNo. \\
M\&D $99-013$
\end{tabular}} \\
\hline $\mathbf{M} \mathcal{E}_{\mathbf{z}} \mathrm{D}^{\text {Professional }}$ & & & \\
\hline \multirow{2}{*}{\multicolumn{2}{|c|}{$\begin{array}{l}\text { Client/Location } \\
\text { ARES Corporation }\end{array}$}} & Prepared B] & \\
\hline & & B. Winkel & \\
\hline \multicolumn{2}{|c|}{$\begin{array}{l}\text { Subject } \\
\text { Floor/Wall Joint Evaluation for Drop Loading }\end{array}$} & & 0 \\
\hline \multicolumn{2}{|c|}{$\begin{array}{l}\text { Ref. Drawing(s) } \\
\mathrm{H}-1-21072\end{array}$} & Revised By 7 Dale & \\
\hline
\end{tabular}

ngen, $2,700,149,195,23,,-16.5$

ngen, $2,700,153,199,23,,-16.5$

fill, 849,853

fill, 872,876

fill, 895,899

e, $826,827,850,849$

egen, $4,1,-1$

egen, $4,23,-4$

e, $126,127,827,826$

egen, $4,1,-1$

$\mathrm{e}, 149,126,826,849$

egen, $4,23,-1$

e, $130,153,853,830$

egen, $4,23,-1$

e, $219,218,918,919$

egen, $4,1,-1$

!generate rebar eles.

ngen, $2,700,49,67,2,, 8.15$

ngen, $2,700,95,113,2,, 8.15$

ngen, $2,700,141,509,46,, 8.15$

ngen, $2,700,143,511,46,, 8.15$

ngen, $2,700,157,525,46,, 8.15$

ngen, $2,700,159,527,46,,, 8.15$

ngen, $2,700,555,573,2,, 8.15$

ngen, $2,700,624,642,2,, 8.15$

real, 7

type, 2

mat, 2

e,49,749

egen, $10,2,-1$

e, 95,795

egen, $10,2,-1$

e, 141,841

egen, $9,46,-1$

e, 143,843

egen, $9,46,-1$

e, 157,857

egen, $9,46,-1$

e, 159,859

egen, $9,46,-1$

e, 555,1255

egen, $10,2,-1$

e, 624,1324

egen, $10,2,-1$

type, 1

mat, 1

!Keypoints

k, $1,0,0,0$

k, $2,14,0,0$

$\mathrm{k}, 3,114,0,0$ $\mathrm{k}, 4,128,0,0$

$k, 5,0,15,88$

k, $6,14,15.88,8.15$

k, $7,114,15.88,8.15$

k. $8,128,15.88$

k, $9,0,147$

$k, 10,14,147,8.15$

k, $11,37.5,147,8.15$

$k, 12,89.0,147,8.15$

$\mathrm{k}, 13,114,147,8.15$

$k, 14,128,147,8.15$

$\mathrm{k}, 15,0,166$,

$k, 16,14,166$

$k, 17,37.5,166$

$k, 18,89.0,166$

k, 19,114,166

k,20,128,166

$\mathrm{k}, 21,14,15.88,63$

$\mathrm{k}, 22,114,15,88,63$

k,23,14,147,63

$k, 24,37.5,147,63$

$k, 25,90.5,147,63$

$\mathrm{k}, 26,114,147,63$

$\mathrm{k}, 27,14,15.88,324$

$k, 28,114,15.88,324$

k, $29,14,147,324$

$\mathrm{k}, 30,37.5,147,324$

k,31,90.5,147,324

k,32,114,147,324

$\mathrm{k}, 33,37.5,215,63$

$\mathrm{k}, 34,90.5,215,63$

$\mathrm{k}, 35,37.5,215,324$

$\mathrm{k}, 36,90.5,215,324$

$\mathrm{k}, 37,37.5,0$

$k, 38,89.0,0 \quad$ !sump edge loca.

$\mathrm{k}, 39,37.5,15,88,8.15$

$\mathrm{k}, 40,89.0,15,88,8.15$

$k, 41,37.5,15,88,63$

$\mathrm{k}, 42,90.5,15.88,63$

$\mathrm{k}, 43,37.5,15.88,324$

$\mathrm{k}, 44,90.5,15.88,324$

$\mathrm{k}, 45,-200,215,63$

$k, 46,-200,215,324$

k,47,250,215,63

$\mathrm{k}, 48,250,215,324$

$k, 49,-200,153.5,63$

$k, 50,37.5,153.5,63$

$k, 51,90.5,153.5,63$

$\mathrm{k}, 52,250,153.5,63$

$k, 53,37.5,153.5,324$

$k, 54,90.5,153.5,324$

$k, 55,-200,335,63$ 
SNF-5470, Revision 0

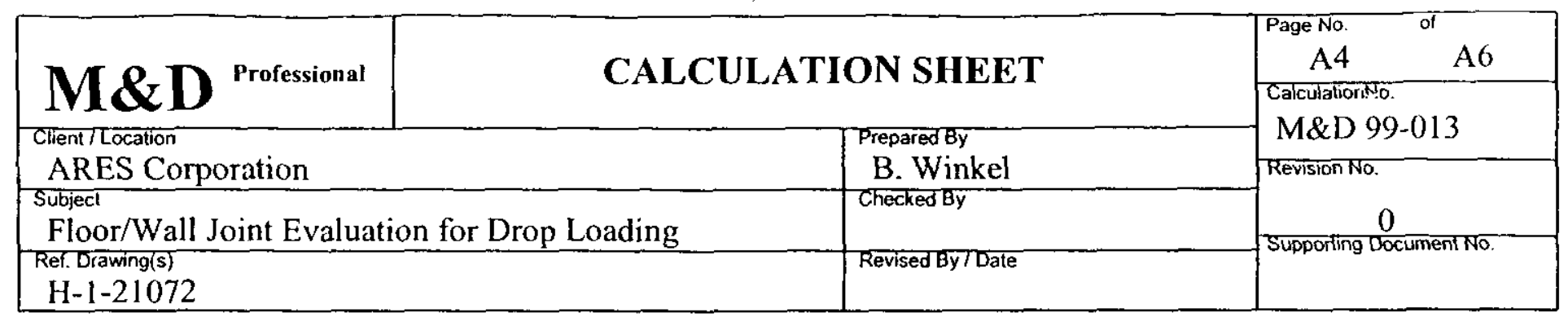

$k, 56,37.5,335,63$

k, $57,90.5,335,63$

$k, 58,250,335,63$

$k, 59,14,15.88,348$

$\mathrm{k}, 60,37.5,15.88,348$

$\mathrm{k}, 61,90.5,15.88,348$

$k, 62,114,15.88,348$

k, $63,14,147,348$

k, $64,37.5,147,348$

k, $65,90.5,147,348$

k, $66,114,147,348$

!Areas

a, $1,2,6,5$

a, $2,37,39,6$

a, $37,38,40,39$

a, $38,3,7,40$

a, $3,4,8,7$

a, $5,6,10,9$

a, $6,39,11,10$

a, $39,40,12,11$

a, $40,7,13,12$

a, $7,8,14,13$

a, $9,10,16,15$

a, $10,11,17,16$

a, $11,12,18,17$

a, 12,13,19,18

a, 13,14,20,19 !Area 15

aatt, 4

asel,none

a, $6,39,41,21$

a, $39,40,42,41$

a, $40,7,22,42$

a, $27,43,60,59$

a, $43,44,61,60$

a,44,28,62,61 !Area 21

aatt, 1

asel,none

a, $10,6,21,23$

a, $13,7,22,26$

a, $29,27,59,63$

a,32,28,62,66 !Area 25

aatt, 2

asel,none

a, $21,41,43,27$

a, $41,42,44,43$

a, $42,22,28,44$

aatt, 1

asel,none

a, $23,21,27,29$

a, $26,22,28,32$

a, $24,23,29,30$ a, 26,25,31,32

a, $33,50,53,35$

a, $50,24,30,53$

a, 34,51,54,36

a,51,25,31,54 !Area 36

aatt, 2

asel,none

$\mathrm{a}, 45,33,35,46$

a, $34,47,48,36$

aatt, 3

asel,none

a, $11,10,23,24$

a, 12,11,24,25

a, $13,12,25,26$

a, $45,49,50,33$

a, $33,50,51,34$

a, $50,24,25,51$

a,34,51,52,47

a, $55,45,33,56$

a, $56,33,34,57$

a,57,34,47,58 !Area 48

aatt, 5

alls

esize, 8.3

eshape, 2

!amesh, 1,7

!amesh,9,15

isel, $\mathrm{s}, \mathrm{loc}, \mathrm{z}, 8.15$

!lesize,all,6.5

alls

esize, 13

amesh, 16,48

!amesh, 16,18

!amesh,22,23

!amesh,39,41

aclear, 19,21

aclear, 24,38

aclear, 42,48

!wall/floor connection plates

real, 9

mat, 3

e, $749,751,1345,1344$

e, $751,753,1343,1345$

e, $753,755,1365,1343$

e, $755,757,1364,1365$

e, $757,759,1363,1364$

e,759,761,1362,1363

e,761,763,1361,1362

e,763,765,1392,1361

e,765,767,1391,1392

e, $795,749,1344,1344$

e,841,795,1344,1434 
SNF-5470, Revision 0

\begin{tabular}{|c|c|c|c|}
\hline $\mathbf{M} \mathcal{C} \mathrm{D}^{\text {Professional }}$ & \multicolumn{2}{|c|}{ CALCULATION SHEET } & \multirow{2}{*}{\begin{tabular}{|c|}
$\begin{array}{c}\text { Page No. } \\
\text { A5 }\end{array}$ \\
CalculationNo. \\
M\&D
\end{tabular}} \\
\hline $\begin{array}{l}\text { Client/Location } \\
\text { ARES Corporation }\end{array}$ & & Prepared By & \\
\hline $\begin{array}{l}\text { ARES Corporation } \\
\text { Subject } \\
\text { Floor/Wall Joint Evalua }\end{array}$ & oading & $\begin{array}{l}\text { B. Winkel } \\
\text { Checked By }\end{array}$ & $\begin{array}{r}\text { Revision No. } \\
0\end{array}$ \\
\hline $\begin{array}{l}\text { Ref. Drawing(s) } \\
\mathrm{H}-1-21072\end{array}$ & & RevisedBy/Dale & Supporting Document No. \\
\hline
\end{tabular}

e,887,841,1434,1435

e, $933,887,1435,1436$

e, $979,933,1436,1437$

e, $1025,979,1437,1438$

e, $1071,1025,1438,1439$

e, $1117,1071,1439,1440$

e, $1163,\{117,1440,1441$

e, 1209,1163,1441,1442

e, $1255,1209,1442,1443$

e, $1255,1443,1433,1324$

e, $1326,1324,1433,3328$

e, $1328,1326,3328,3327$

e, $1330,1328,3327,3341$

e, 1332,1330,3341,3340

e, $1334,1332,3340,3339$

e, $1336,1334,3339,3338$

e, $1338,1336,3338,3337$

e, $1340,1338,3337,3366$

e, 1342,1340,3366,1499

e, 1273,1342,1499,1509

e, $1273,1509,1508,1227$

e, $1227,1508,1507,1181$

e, $1181,1507,1506,1135$

e, $1135,1506,1505,1089$

e, 1089,1505,1504,1043

e, $1043,1504,1503,997$

e, $997,1503,1502,951$

e,951,1502,1501,905

e,905,1501,1500,859

e, $859,1500,1391,813$

e,767,813,1391,1391

$\mathrm{e}, 1344,1345,797,797$

e, 1345,1343,799,797

$\mathrm{e}, 1343,1365,801,799$

$\mathrm{e}, 1365,1364,803,801$

e, $1364,1363,805,803$

e, $1363,1362,807,805$

$\mathrm{e}, 1362,1361,809,807$

e,1361,1392,811,809

e,1392,1391,811,811

$\mathrm{e}, 1344,797,843,1434$

$\mathrm{e}, 1434,843,889,1435$

e, $1435,889,935,1436$

$\mathrm{e}, 1436,935,981,1437$

e, 1437,981,1027,1438

e, 1438,1027,1073,1439

e, $1439,1073,1119,1440$

$\mathrm{e}, 1440,1119,1165,1441$

$\mathrm{e}, 1441,1165,1211,1442$

e, 1442,1211,1257,1443

e, $1443,1257,3328,1433$ e, 1257,1259,3327,3328

e,1259,1261,3341,3327

e, $1261,1263,3340,3341$

e, $1263,1265,3339,3340$

e, $1265,1267,3338,3339$

e, $1267,1269,3337,3338$

e, $1269,1271,3366,3337$

e,1271,1509,1499,3366

e, 1271,1225,1508,1509

$\mathrm{e}, 1225,1179,1507,1508$

e, $1179,1133,1506,1507$

e, $1133,1087,1505,1506$

e, 1087,1041,1504,1505

e, $1041,995,1503,1504$

e, $995,949,1502,1503$

$\mathrm{e}, 949,903,1501,1502$

$\mathrm{e}, 903,857,1500,1501$

e,857,811,1391,1500

!Gap elements on outside rebar

real, 10

type, 3

e,49,749

egen, $10,2,-1$

e, 624,1324

egen, $10,2,-1$

e,95,795

egen, $11,46,-1$

$\mathrm{e}, 113,813$

egen, $11,46,-1$

nummrg,all

n,4192,38.251,45.012,6

$\mathrm{n}, 4200,89.749,45.012,6$

$\mathrm{n}, 4330,38.251,79.998,6$

n, 4338,89.749,79.998,6

!impacting mass

$\mathrm{n}, 4400,64,62.5,6$

$\mathrm{n}, 4401,64,62.5,12$

type, 6

real, 13

$\mathrm{e}, 4401$ !mass

type, 3

real,14

type, 5

real, 17

e,4400,4401 !nonlinear spring (impact limiter)

e,192,4192 !gap elements

$\mathrm{e}, 200,4200$

e, 330,4330

e, $, 338,4338$ 
SNF-5470, Revision 0

\begin{tabular}{|c|c|c|c|}
\hline Mde $D^{\text {Professional }}$ & \multicolumn{2}{|c|}{ CALCULATION SHEET } & \multirow{2}{*}{\begin{tabular}{|l}
$\begin{array}{c}\text { Page No. } \\
\text { A6 } 6\end{array}$ \\
CalculationNo. \\
M\&D 99-013
\end{tabular}} \\
\hline \multicolumn{2}{|l|}{$\begin{array}{l}\text { Client/Location } \\
\text { ARES Corporation }\end{array}$} & \begin{tabular}{|l} 
Prepared By \\
B. Winkel
\end{tabular} & \\
\hline \multicolumn{2}{|c|}{$\begin{array}{l}\text { Subject } \\
\text { Floor/Wall Joint Evaluation for Dron Loading }\end{array}$} & Checked By & Revision ivo. \\
\hline \multicolumn{2}{|c|}{ Ref. Drawing(s) } & Revised By/Date & Supporing Document No. \\
\hline
\end{tabular}

type, 7

real, 15

e, 192,4192 !dashpots

$\mathrm{e}, 200,4200$

e, 330,4330

$\mathrm{e}, 338,4338$

type, 4

real, 16

e,4192,4400 !rigid links

$\mathrm{e}, 4200,4400$

e, 4330,4400

$\mathrm{e}, 4338,4400$

/VIEW, $1,0.126685636958,-0.479200338424 \mathrm{E}-01$, 0.99078474945 l

/ANG, 1, -61.5345416226

eplo

!Water Pressure Loading

esel,s,real, 4

nsle

nsel,s,loc,y,15.8,147.1

nsel, , loc, $\mathrm{x}, 13.9,114.1$

nsel,r,loc, $z, 0$

esln, 1

sfe,all,,pres,,-9.75 !pit floor water pressure

alls

nsel,s,loc, $z, 62.9,63.1$

d,all,uy,,,,ux,uz

nsel,s,node, ,4000,4500

d,all,ux,.,., uy

nall

d, 1, ux,,.,,uy

d, 23 ,uy

fini

/solu

antype,trans

outres,all,all

timint, off

$\mathrm{d}, 4401, u z,-.3446$

d, $4401, u x$

d, 4401 ,uy

time, 0.001

nsubst, 4

solve

ddel,4401,uz

timint,on

time,. 05

deltim,.00005,.0000],.001

!initial veloc. solve

fini

/post26

nsol, 2,4401,u,z,uzmass

deriv, $3,2,1$, ,vzmass

plvar,2

ESOL,4,2791, ,SMIS, 1,FZ

PLVAR, 4

NSOL,5,57,U,Z,uz57

plvar, 5 
SNF-5470, Revision 0

\begin{tabular}{|c|c|c|c|}
\hline $\mathbf{M} \boldsymbol{E} \mathbf{D}^{\text {Professional }}$ & \multicolumn{2}{|c|}{ CALCULATION SHEET } & $\begin{array}{l}\begin{array}{l}\text { Page No. } \\
\text { B I }\end{array} \\
\text { Calculationino. }\end{array}$ \\
\hline \multicolumn{2}{|l|}{$\begin{array}{l}\text { Client/Location } \\
\text { ARES Corporation }\end{array}$} & $\begin{array}{l}\text { PreparedBy } \\
\text { B. Winkel }\end{array}$ & $\begin{array}{l}\text { M\&D 99-013 } \\
\text { Revision No. }\end{array}$ \\
\hline \multicolumn{2}{|c|}{$\begin{array}{l}\text { Subject } \\
\text { Floor/Wall Joint Evaluation for Drop Loading }\end{array}$} & & 0 \\
\hline \multicolumn{2}{|c|}{$\begin{array}{l}\text { Rel Drawing(s) } \\
\mathrm{H}-1-21072\end{array}$} & Revised By/Date & \\
\hline
\end{tabular}

APPENDIX B

BENCHMARK TESTING OF ANALYSIS METHODOLOGY 
SNF-5470, Revision 0

\begin{tabular}{|c|c|c|c|}
\hline M $g_{2}$ Professional & \multicolumn{2}{|c|}{ CALCULATION SHEET } & $\begin{array}{|cc|}\text { Page No. } & \text { of } \\
\text { B2 } & \text { B4 } \\
\text { CalculationNo. }\end{array}$ \\
\hline \multirow{2}{*}{\multicolumn{2}{|c|}{$\begin{array}{l}\text { Client/Location } \\
\text { ARES Corporation }\end{array}$}} & Prepared By & M\&D 99-013 \\
\hline & & B. Winkel & Revision No. \\
\hline \multicolumn{2}{|c|}{$\begin{array}{l}\text { Subject } \\
\text { Floor/Wall Joint Evaluation for Drop Loading }\end{array}$} & Checked By & 0 \\
\hline \multicolumn{2}{|c|}{$\begin{array}{l}\text { Rer Drawing(s) } \\
\mathrm{H}-\mathrm{I}-21072\end{array}$} & Revised By/Date & \\
\hline
\end{tabular}

\subsection{INTRODUCTION}

The purpose of this appendix is to address the adequacy of the dynamic analysis methodology used in evaluating the structural response to a drop onto the $105 \mathrm{~K}$ fuel storage basin floor. The basis for addressing the methodology adequacy is by analytically duplicating impact testing performed at Sandia Laboratories (Reference 15) and comparing the analytical results with the test data. By appropriately modifying the analytical model parameters, the analytical approach can be tuned to the test data and the resulting modeling/methodology is "benchmarked" against actual drop tests which are similar to the fuel storage basin drop accident configurations.

\subsection{LOW VELOCITY IMPACT TEST PROGRAM REVIEW}

Reference 15 summarizes the results from a series of four low velocity impact testing programs performed at Sandia and Lawrence Livermore Laboratories. All of the tests involved steel billets (weight: 6475 and 6600 lbs.) dropping onto 12-in and 18-in. thick concrete pads. The drop heights ranged from 18 in. to 72 in. Tests \#233 and \#234 of Test Series No. 3 involve 72-in. end drops of 6600-lb. billets onto a 1-ft. thick concrete $\mathrm{slab}$, which is closest to the $3400-\mathrm{lb}$. fuel basket drop of $8-\mathrm{ft}, 1$-in. These two test configurations were selected to benchmark the drop analysis approach used in the $\mathrm{K}$ Basin floor drop evaluations.

For the tests of interest (\#233 and \#234) the steel billets were instrumented with six accelerometers--four on the top and two on the side of each billet. Acceleration time histories are reported for all six instrumented locations.

\subsection{BENCHMARK TEST ANALYSIS MODEL}

The benchmark test configuration involved a 6 -ft. by $6-\mathrm{ft}$. slab, with a thickness of $1 \mathrm{ft}$. The slab for test \#233 was supported with a 1 - $\mathrm{ft}$. thick "engineered fill" on top of an "unknown soil B". The test \#234 slab was supported directly on grade ("unknown soil B"). Consistent with the K Basin drop analysis approach, the concrete slab was modeled using shell elements (ANSYS SHELL63). The ANSYS model is shown in Figure B-1. The 6600-lb. billet was modeled with a single mass element (ANSYS MASS21), which was given an impact velocity of $236 \mathrm{in.} / \mathrm{sec}$. (72-in. drop). The interface between the mass and the slab included a gap element (ANSYS CONTAC52) and a damper (ANSYS COMBIN14).

The slab was supported on elastic springs using a lower-bound subgrade modulus of $100 \mathrm{psi} / \mathrm{in}$. An upperbound modulus of $500 \mathrm{psi} / \mathrm{in}$. was also considered, but the predicted mass decceleration was found to be relatively insensitive to the soil stiffness. The peak predicted mass deccelerations were found to be relatively sensitive to the mass/slab interface stiffness and damper constants. A range of interface element parameters were considered, as described below. Consistent with the analysis approach described in Section 4.1.2, the damper dashpot constant magnitude corresponded to the critical damping value $\left(\mathrm{c}=2(\mathrm{~km})^{1 / 2}\right)$. 
SNF-5470. Revision 0

\begin{tabular}{|c|c|c|c|}
\hline M\&d $\mathbf{D}^{\text {Professional }}$ & \multicolumn{2}{|c|}{ CALCULATION SHEET } & \multirow{2}{*}{\begin{tabular}{|l|}
$\begin{array}{c}\text { Page No. } \\
\text { B3 }\end{array}$ \\
CalculationNo. \\
M4 \\
M\&D 99-013 \\
\end{tabular}} \\
\hline $\begin{array}{l}\text { Chent/location } \\
\text { ARES Corporation }\end{array}$ & & $\begin{array}{l}\text { Prepared By } \\
\text { R Winkel }\end{array}$ & \\
\hline $\begin{array}{l}\text { Subject } \\
\text { Floor/Wall Joint Evalua }\end{array}$ & oading & Checked By & 0 \\
\hline $\begin{array}{l}\text { Ref. Drawing(s) } \\
\mathrm{H}-1-21072\end{array}$ & & Revised By / Date & \\
\hline
\end{tabular}

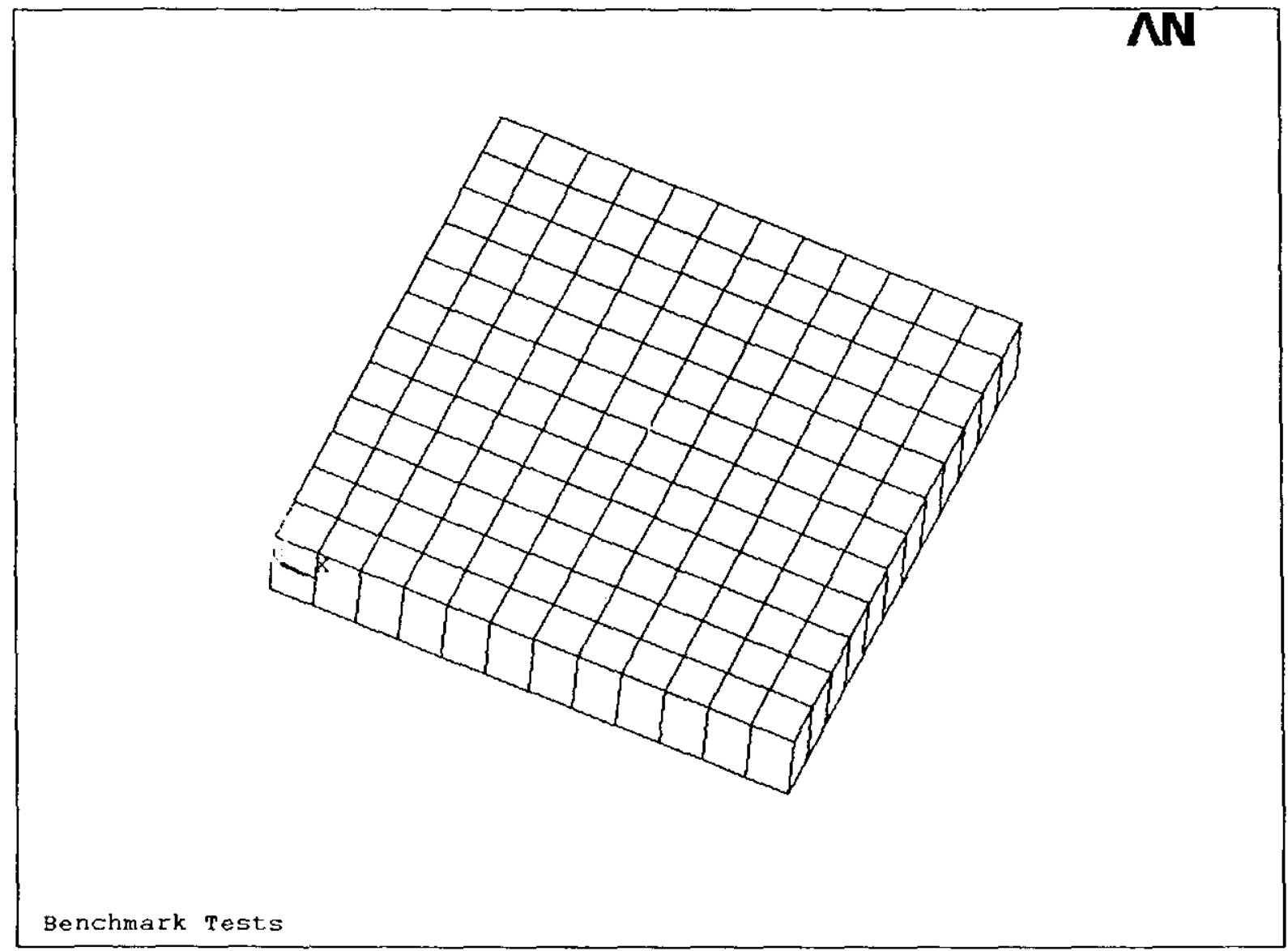

Figure B-1 - Finite Element Model of Benchmark Test Configuration

\subsection{COMPARISON OF ANALYSIS AND TEST RESULTS}

The predicted mass decceleration histories for various values of interface stiffness are shown in Figure B-2. The peak deccelerator values for the 12 reported test data sets ranged from 121 to $210 \mathrm{~g}$ 's. The "Sandia Tests" curve shown represents the upper bound test results for test \#233 and \#234. Note that a model impact stiffness of $1 \times 10^{6} \mathrm{lb}$./in. predicts a peak acceleration above the bounding test data.

\subsection{CONCLUSION}

Based upon the analytical/test data comparisons described above, the most important model parameters for duplicating the Sandia drop test data are the interface stiffness and damper constant. By assuming critical damping and a model interface stiffness of $1 \times 10^{6} \mathrm{lb}$./in., a reasonable upper bound to the test data can be obtained. 
SNF-5470, Revision 0

\begin{tabular}{|c|c|c|c|}
\hline $\mathbf{M} \boldsymbol{\&} \mathbf{D}^{\text {Professional }}$ & \multicolumn{2}{|c|}{ CALCULATION SHEET } & \multirow{2}{*}{ 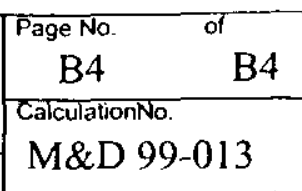 } \\
\hline $\begin{array}{l}\text { Chent/Location } \\
\text { ARES Cornoration }\end{array}$ & & Prepared By & \\
\hline $\begin{array}{l}\text { ARES Corporation } \\
\text { Subject } \\
\text { Flor/Wall Ioint Fylu }\end{array}$ & 07 & $\begin{array}{l}\text { B. Winkel } \\
\text { Checked By }\end{array}$ & $\begin{array}{r}\text { Revision No. } \\
0\end{array}$ \\
\hline $\begin{array}{l}\text { Floor/Wall Jont Evasuat } \\
\text { Refrawing(s) } \\
\text { - } 1-21072\end{array}$ & & Revised By/Date & Supporting Docuinent No. \\
\hline
\end{tabular}

\section{Analysis/Test Comparison}

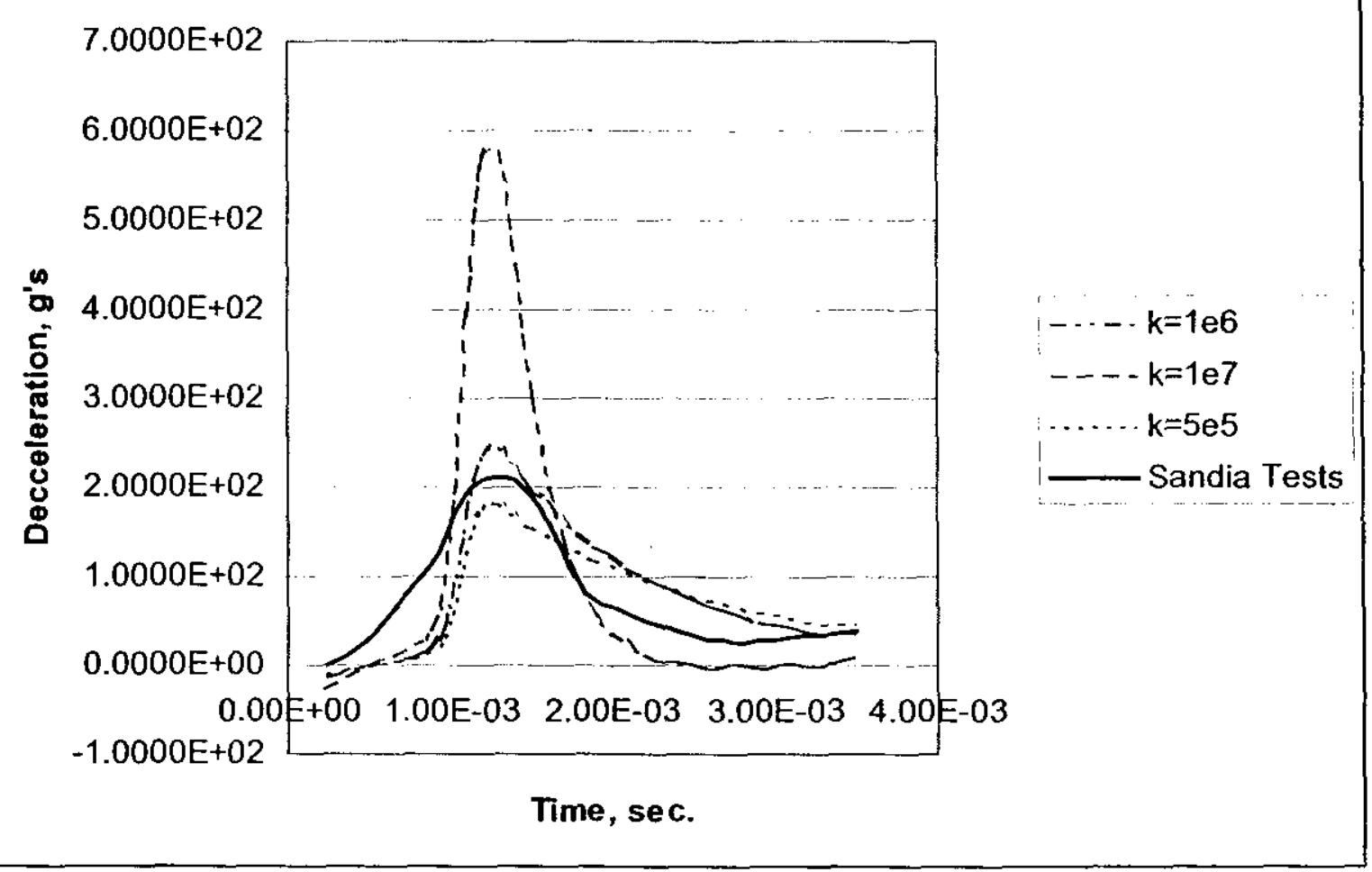

Figure B-2 - Comparison of Drop Test Data with Analytical Predictions 


\begin{tabular}{|c|c|}
\hline 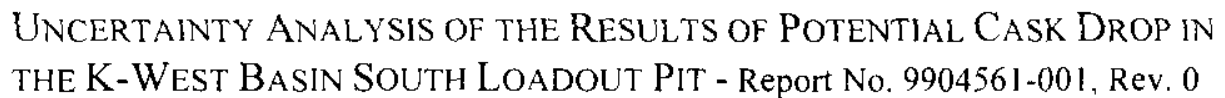 & $\begin{array}{r}\text { SNF-5470, Revision } 0 \\
\text { November } 1999\end{array}$ \\
\hline
\end{tabular}

Appendix C

Impact Energy and Associated Concrete Crack Diameter 


\section{C.1 Introduction}

In Appendix B the residual gap diameter between the SLOP basin floor and wall was determined for a range of concrete impact energies. The analysis used both best engineering values as well as conservative values for the parameters used in the analysis over the range of concrete impact energies. In this Appendix the results of Appendix B are combined with uncertainty distributions to determine the distribution of the residual gap diameters between the SLOP basin floor and wall. These residual gap diameters are used in Appendix $D$ to determine the initial flow rate through this gap.

\section{C.2 Uncertainty in the Residual Gap Diameter}

The results of Appendix B, Table 5-1, are graphed in Figure C-1 as the residual floor/wall gap diameter as a function of the concrete impact energy for the analyses using both the best engineering and conservative values of the parameters. The results for each set of data are reasonably linear, specifically in light of the uncertainty in the analysis. Therefore, each set of data were fit to a linear expression. The fitted linear curves as well as the 5 th and 95 th percent confidence levels for the fitted curves are given in Figure C-1.

The uncertainty in the residual floor/wall gap diameter given by the curves for the analyses using best engineering and conservative parameters was assumed to be lognormally distributed. The curve representing the results of the analysis using best engineering parameters was assumed to be the median value of the Lognormal distribution. The Lognormal standard deviation was varied until the spread in the distribution enveloped the results of the analysis using the conservative parameters. This process was repeated for each set of data given in Figure C-1. As the median value of the Lognormal distribution decreased (as represented by the curve that represents the results using best engineering parameters) the spread in the distribution also decreased such that a constant Lognormal standard deviation appeared to represent all of the data. Assuming a constant Lognormal standard deviation while varying the median value of the Lognormal distribution simplifies the analysis to follow. The results for the distribution of residual floor/wall gap as a function of the impact energy is shown in Figure C-2. The over-laid Lognormal distribution in Figure C-2 represents the uncertainty in the floor/wall gap at each concrete impact energy.

The information in Figure $\mathrm{C}-2$ is used as follows. For each value of the concrete impact energy the median value of the distribution for the floor/wall gap is determined using the linear equation that represents the results in Figure $\mathrm{C}-2$ using best engineering parameters. This median value and the constant standard deviation represents a Lognormal distribution from which a value of the floor/wall gap is determined. This process is repeated for 10,000 iterations, each time selecting a new impact energy, a median value of a Lognormal distribution for the floor/wall gap 
UnCERTAinty ANalysis of the Results of Potential CaSk Drop in

SNF-5470, Revision 0

THE K-WEST BASIN SOUTH LOADOUT PIT - Report No. 9904561-001. Rev. 0

November 1999

and a value from the distribution for the floor/wall gap.

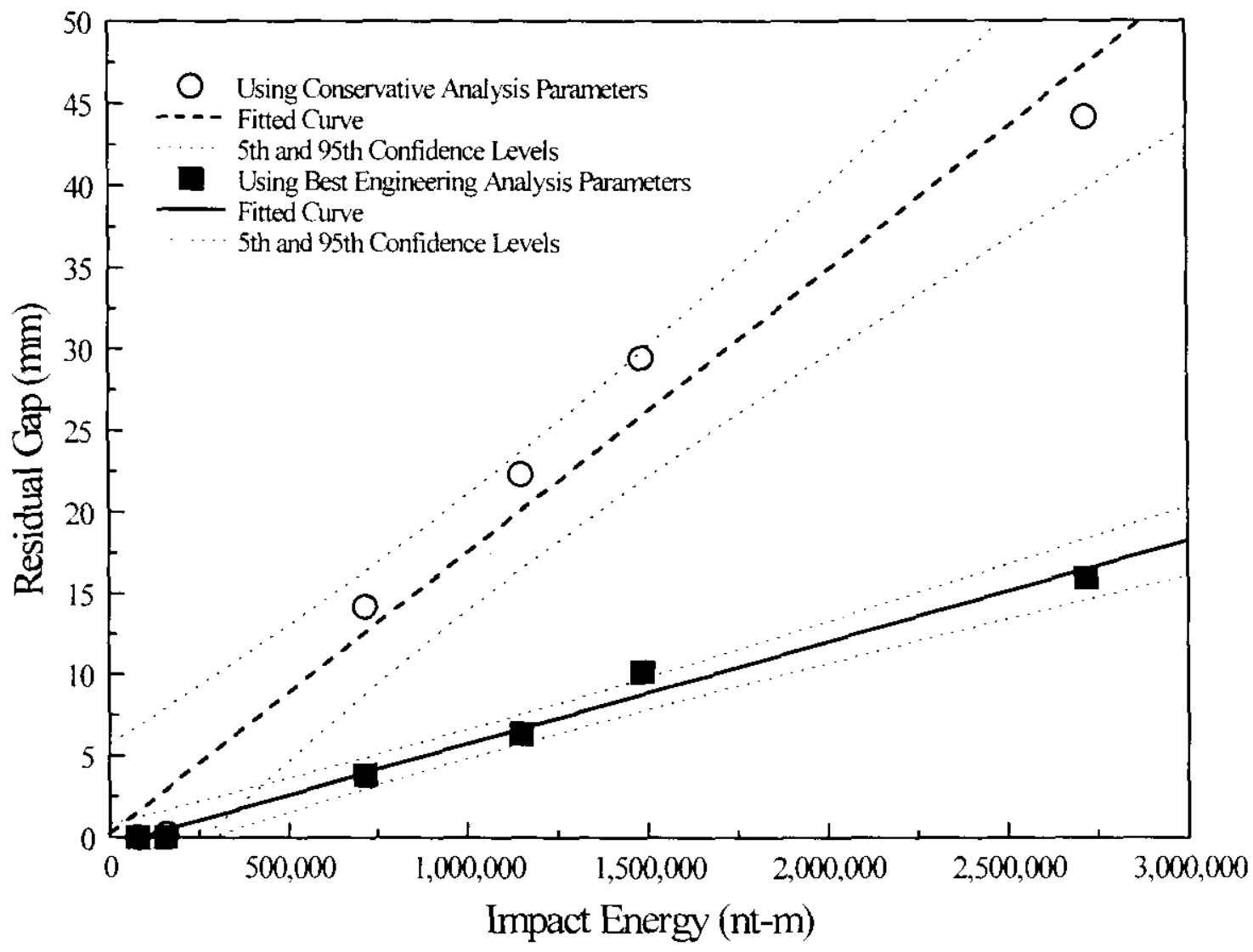

Figure C-1. Residual Floor / Wall Gap às a Function of the Impact Energy. 


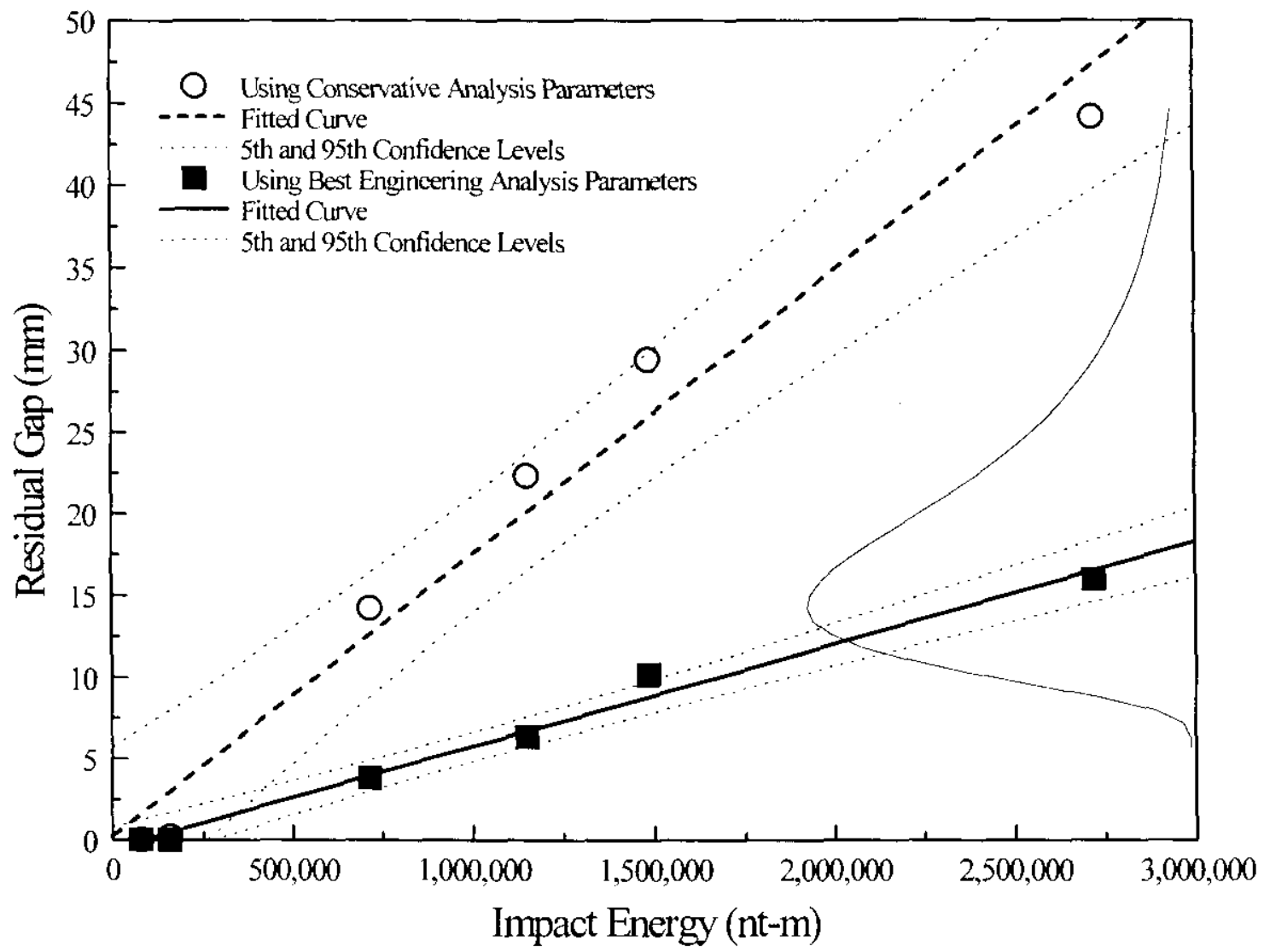

Figure C-2. Uncertainty in the Residual Gap as a Function of the Impact Energy.

The results of the analysis are presented in Figures $\mathrm{C}-3$ and $\mathrm{C}-4$. Figure $\mathrm{C}-3$ is the cumulative distribution function which represents the probability of not exceeding a specific floor/wall gap diameter in millimeters. Figure C-4 is the complementary cumulative distribution function which represents the probability of exceeding a specific floor/wall gap diameter. The most likely floor/wall gap diameter is $4.5 \mathrm{~mm}(0.177 \mathrm{in})$. There is a probability of 0.95 that the floor/wall gap diameter will not exceed $12 \mathrm{~mm}(0.472 \mathrm{in})$. Conversely, there is a probability of 0.05 that the floor/wall gap diameter will exceed $12 \mathrm{~mm}(0.472 \mathrm{in})$. There is a probability of 0.99 that the floor/wall gap diameter will not exceed $18 \mathrm{~mm}(0.709 \mathrm{in})$. These results are used in the analysis of Appendix D. 


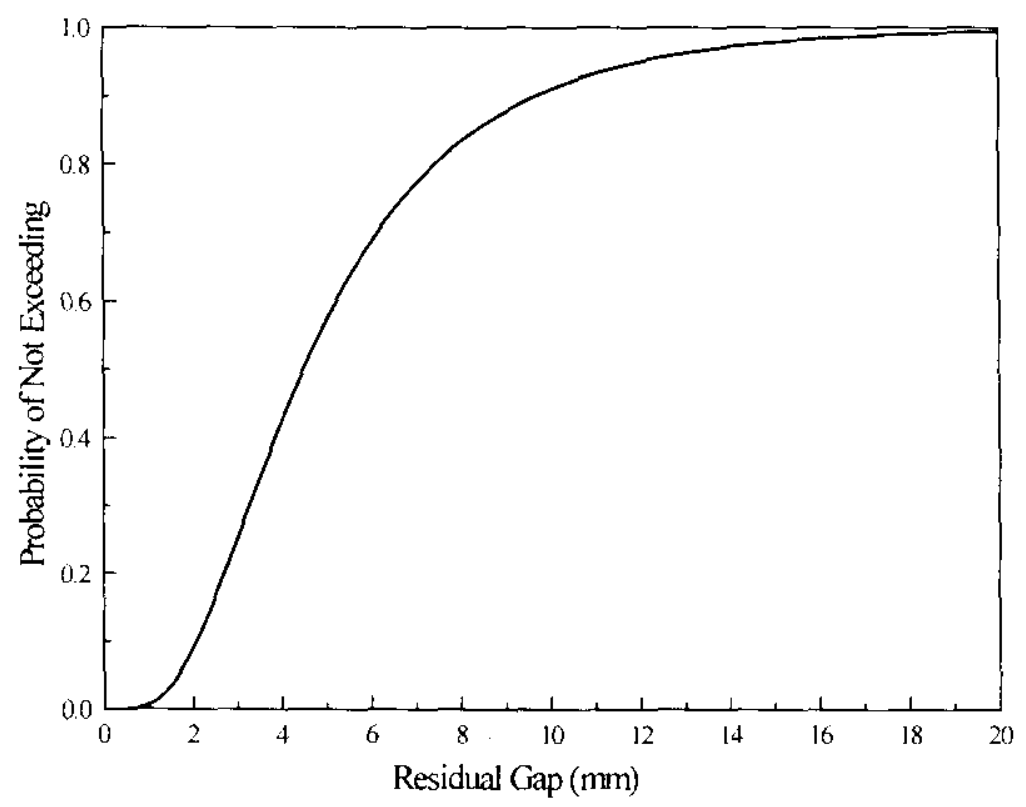

Figure C-3. Cumulative Distribution Function of the Residual Floor/Wall Gap.

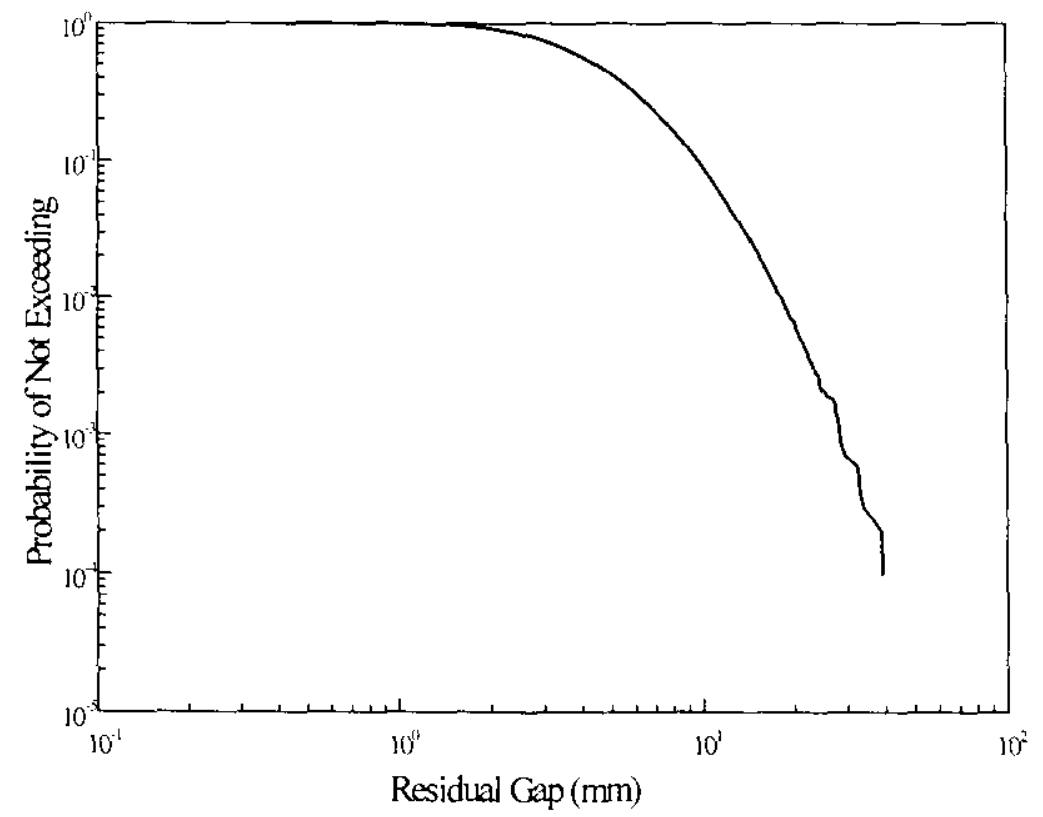

Figure C-4. Complementary Cumulative Distribution Function of the Residual Floor/Wall Gap. 
Appendix D

Flow In A Porous Media 


\section{D.1 Introduction}

This appendix evaluates the volumetric flow rate through the floor/wall gap created by an impact energy to the SLOP basin concrete floor from dropping a cask and MCO. The analysis starts with fluid flow which in general is given by the expression $\mathrm{Q}=\mathrm{A} \mathrm{U}$, where $\mathrm{A}$ is the crossectional area of the floor/wall gap and $U$ is the superficial fluid velocity. The pressure gradient through the floor/wall gap is expressed as $\Delta P=f(U)$. The pressure gradient through the floor/wall gap is defined due to various conditions as a function of the superficial velocity and other physical parameters. The superficial velocity is determined from the pressure gradient, and is used to evaluate the fluid flow. In this appendix the equations are expressed in SI units, but the final results will be expressed in English units.

\section{D.2 Early Analysis}

In a series of letter reports Plys (Plys 1999) estimated the initial leak rate from the SLOP due to an assumed drop of the cask. The initial leak rate was through the floor/wall joint that was separated due to the impact energy on the concrete floor. The first estimate considered tubular flow through the floor/wall gap. The volumetric flow rate was given by the expression;

$$
\frac{\mathrm{Q}}{\mathrm{A}}=\mathrm{C}_{\mathrm{D}} \sqrt{2 \overline{\mathrm{g} \mathrm{H}}} \text {, }
$$

where $\mathrm{Q}=$ volumetric flow rate in $\mathrm{m}^{3} / \mathrm{sec}$,

$\mathrm{C}_{\mathrm{D}}=$ loss coefficient,

$\mathrm{g}=$ gravitational acceleration $\left(9.81 \mathrm{~m}^{2} / \mathrm{sec}\right)$, and

$\mathrm{H}=$ water depth.

The loss coefficient is approximated by the expression;

$$
C_{D}=\frac{1}{\sqrt{K+\frac{4 f L}{D}}},
$$

where $\mathrm{K}=$ total loss coefficient for acceleration, contraction, expansion, etc,

$\mathrm{f}=$ friction factor,

$\mathrm{L}=$ length of the flow path, and

$\mathrm{D}=$ equivalent hydraulic diameter which is $2 \times$ gap thickness.

In this analysis, there were discussions about the concrete path roughness, $\epsilon$, and friction factors. Ranges of $\epsilon$ were provided $(0.3$ to $3 \mathrm{~mm})$ from which ranges of $\epsilon / \mathrm{D}$ could be estimated. 
In the second analysis (Plys 1999) consideration was given to estimating the initial leak rate through the floor/wall gap at the SLOP floor wall joint using a porous medium approach. It was presented that a porous medium approach was applicable because the roughness $(\epsilon)$ is as large or larger than the floor/wall gap thickness. Plys notes that there is an experimental basis for application of the porous medium approach to correlate flow through cracks in reinforced concrete (Rizkalla, et al. 1979).

The pressure gradient in a porous medium is given by (Macdonald, et al. 1979)

$$
\frac{\Delta \mathrm{P}}{\mathrm{L}}=\frac{180(1-\epsilon)^{2}}{\mathrm{D}_{\mathrm{n}}^{2} \epsilon^{3}} \mu \mathrm{U}+\frac{\mathrm{B}(1-\epsilon)}{\mathrm{D}_{\mathrm{n}} \epsilon^{3}} \rho \mathrm{U}^{2},
$$

where $\Delta \mathrm{P}=$ pressure drop, which is given by $\Delta \mathrm{P}=\rho \mathrm{g} \mathrm{H}(\mathrm{Pa})$,

$\mathrm{L}=$ the length for the flow through the floor/wall gap $(\mathrm{m})$,

$\rho=$ water density $\left(\mathrm{kg} / \mathrm{m}^{3}\right)$,

$\mathrm{g}=$ gravitational acceleration $\left(9.81 \mathrm{~m} / \mathrm{s}^{2}\right)$,

$\mathrm{H}=\mathrm{K}$ Basin water height $(7.4 \mathrm{~m})$,

$D_{n}=$ equivalent average flow diameter $(m)$,

$\epsilon=$ porosity

$\mu=$ viscosity $\left(1 \times 10^{-3} \mathrm{~kg} / \mathrm{m}\right.$-sec $)$,

$\mathrm{U}=$ superficial velocity $(\mathrm{m} / \mathrm{sec})$,

$A=$ flow area $\left(\mathrm{m}^{2}\right)$, and

$\mathrm{B}=$ roughness parameter.

Plys noted that the roughness parameter ranges from 2 for smooth particles to 4 for rough particles in a porous medium.

In a third analysis Epstein (Plys 1999) included in the equation for the pressure gradient in a porous medium a contribution for the flow resistance imposed by the soil just out side of the floor/wall gap. For this case the pressure gradient from the floor/wall gap inlet region to the soil outside and downstream of the gap outlet is given by;

$$
\Delta \mathrm{P}=\frac{180(1-\epsilon)^{2}}{\mathrm{D}_{\mathrm{n}}^{2} \epsilon^{3}} \mu \mathrm{LU}+\frac{\mathrm{B}(1-\epsilon)}{\mathrm{D}_{\mathrm{n}} \epsilon^{3}} \rho L U^{2}+\frac{2 \mu \mathrm{D}_{\mathrm{n}} \mathrm{U}}{\kappa_{\text {soil }}}
$$

where $\kappa=$ the soil permeability.

The last term in Equation D-4 represents the pressure drop in the exterior soil. The soil permeability is related to the saturated hydraulic conductivity by the expression; 


$$
\kappa_{\text {soil }}=\frac{\mu \mathrm{k}_{\text {soil }}}{\rho \mathrm{g}} .
$$

Therefore Equation D-4 becomes;

$$
\Delta P=\frac{180(1-\epsilon)^{2}}{D_{n}^{2} \epsilon^{3}} \mu L U+\frac{B(1-\epsilon)}{D_{n} \epsilon^{3}} \rho L U^{2}+\frac{2 \rho g D_{n} U}{k_{\text {sois }}} .
$$

Note that in Equation D-6 the first term is a viscous pressure drop, the second term in an inertial pressure drop, and the third term is a pressure drop due to the resistance of the exterior soil. The viscous pressure drop varies as $1 / D_{n}{ }^{2}$, the inertial pressure drop varies as $1 / D_{n}$, while the pressure drop due to the exterior soil resistance varies as $\mathrm{D}_{\mathrm{n}}$. Thus, as the equivalent diameter increases the viscous pressure and inertial pressure decrease while the pressure drop due to the exterior soil resistance increases.

\section{D-3 Considerations of the Flow Through a Porous Medium}

Equation D-6 is a quadratic equation in the superficial velocity U. Equating the pressure gradient as $\Delta \mathrm{P}=\rho \mathrm{g} \mathrm{H}$, the quadratic equation can be written as;

$$
\begin{aligned}
& a U^{2}+b U+c=0 \quad \text { with } \\
& U=\frac{-b \pm \sqrt{b^{2}-4 a c}}{2 a}
\end{aligned}
$$

where

$$
\begin{aligned}
& \mathrm{a}=\frac{\mathrm{B}(1-\epsilon) \rho L}{\mathrm{D}_{\mathrm{n}} \epsilon^{3}} \\
& \mathrm{~b}=\frac{180(1-\epsilon)^{2} \mu L}{\mathrm{D}_{\mathrm{n}}{ }^{2} \epsilon^{3}}+\frac{2 \rho g D_{n}}{\mathrm{k}_{\text {soil }}} \\
& \mathrm{c}=-\rho g \mathrm{H} .
\end{aligned}
$$

The volumetric flow is given by $Q=A U$. Note that $D_{n}$ is the equivalent hydraulic diameter which is 2 times the floor/wall gap thickness. 
The asymmetric impact location of a dropped cask on the K Basin SLOP concrete floor results in the largest separation of the floor/wall joint in the wall nearest to the drop location (west side of the SLOP floor). The separation of the floor/wall joint is close to zero on the east end of the SLOP floor (nearest to the $\mathrm{K}$ Basin entrance). Besides the asymmetric impact location this asymmetry is also due to larger rebar on the east side of the floor/wall joint (see Appendix B). Figure D-1 provides a layout of the K Basin SLOP both in plan view and in a section view. The section view in Figure D-1 shows that the floor/wall gap around the perimeter of the SLOP is approximated by two triangles on the north and south walls and a rectangle on the west wall. Therefore the area of the floor/wall gap is approximated by;

$$
A=2\left(\frac{1}{2} \text { base } * \text { height }\right)+\text { width } * \text { height }=(b+w) * d .
$$

In Equation D-9, $b$ is the base of the SLOP, $w$ is the width of the SLOP, and $d$ is the height of the floor/wall gap. Note that the sum of the base and the width is just equal to the perimeter of the SLOP. As noted in Figure D-1 there is a water stop located midway in the concrete wall that was used as a flow limiter in fabrication of the floor wall joint. For this analysis, the equivalent hydraulic flow area is taken to be the area of the floor/wall gap at the location of the water stop. Therefore, the dimensions used to calculate the perimeter of the SLOP are taken from Figure D-1 and are given by;

$$
\begin{aligned}
& \text { base }=10^{\prime}-111 / 8^{\prime \prime}=131.125-\text { in }=3.3 \mathrm{~m}, \\
& \text { width }=8^{\prime}-6^{\prime \prime}=102-\text { in }=5.6 \mathrm{~m} \text {. } \\
& \text { perimeter = base } x \text { height }=233 \mathrm{in}=5.92 \mathrm{~m} \text {, and } \\
& d=\text { floor } / \text { wall joint gap distance. }
\end{aligned}
$$

Note in Equation D-4, $D_{n}=2 d$.

The volumetric flow rate is given by $Q=A U=p d U=p\left(D_{n} / 2\right) U$. The contribution to the volumetric flow rate due to the pressure drop of the exterior soil is approximated by;

$$
\mathrm{Q} \sim \mathrm{p} \frac{\mathrm{D}_{\mathrm{n}}}{2} \frac{\mathrm{k}_{\text {soil }} \Delta \mathrm{P}}{2 \rho \mathrm{g} \mathrm{D}_{\mathrm{n}}} \sim \frac{\mathrm{D}_{\mathrm{n}}}{\mathrm{D}_{\mathrm{n}}} .
$$

Equation D-10 shows that the resistance to the flow due to the presence of the exterior soil against the SLOP basin wall soon asymptotes to a constant value independent of the floor/wall joint gap.

The contribution of the inertial pressure drop to the volumetric flow rate is approximated by;

$$
\mathrm{Q} \sim \mathrm{p} \frac{\mathrm{D}_{\mathrm{n}}}{2} \sqrt{\frac{\Delta \mathrm{P} \epsilon^{3}}{\mathrm{~B}(1-\epsilon) \rho \mathrm{L}} \mathrm{D}_{\mathrm{n}}} \sim \mathrm{D}_{\mathrm{n}}^{\frac{3}{2}} .
$$



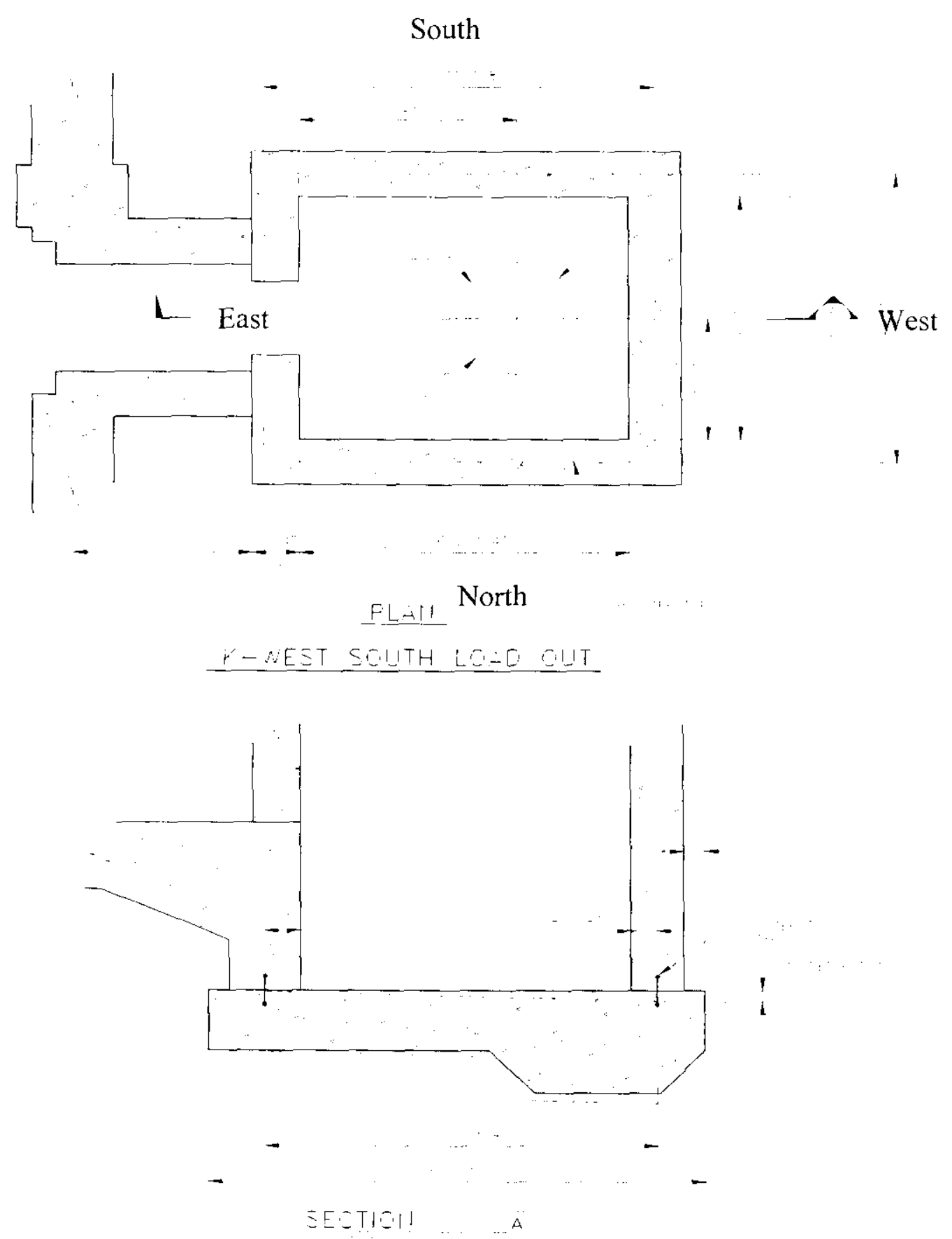

Figure D-1. Layout of the K-West Basin SLOP. 
Similarly the contribution of the viscous pressure drop to the volumetric flow rate is approximated by;

$$
\mathrm{Q} \sim \mathrm{p} \frac{\mathrm{D}_{\mathrm{n}}}{2} \frac{\Delta \mathrm{P} \epsilon^{3}}{180(1-\epsilon)^{2} \mu \mathrm{L}} \mathrm{D}_{\mathrm{n}}^{2} \sim \mathrm{D}_{\mathrm{n}}{ }^{3}
$$

For small floor/wall gap distances, the contribution to the volumetric flow rate is dominated by the inertial pressure drop term. The viscous pressure drop term is very small but increases approximately as $D_{n}{ }^{3}$. However, for large floor/wall gap distances the contribution to the volumetric flow rate is dominated by the pressure drop of the exterior soil which asymptotes to a constant value. Therefore, the volumetric flow rate, when all of the contributions in Equation D6 are included, asymptotes to a constant value for floor/wall gap distances beyond $5 \mathrm{~mm}(0.197$ in) even for largely varying values of the soil permeability.

Point-values for each of the parameters included in Equation D-6 are given in Table D-1. Based on these point-values, the superficial velocity, $U$, was calculated solving the quadratic equation, Equation D-6. Using this superficial velocity, the volumetric flow rate was calculated for a range of floor/wall gap distances. The results are presented in Table D-2 for floor/wall gap distance up to $10 \mathrm{~mm}(0.394 \mathrm{in})$.

Table D-1. Point-Values for Each of the Parameters Listed in Equation D-6

\begin{tabular}{|l|c|c|}
\hline Parameter & Parameter Point Value & Units \\
\hline Viscosity, $\mu$ & $1.0 \times 10^{-3}$ & $\mathrm{~kg} / \mathrm{m}-\mathrm{sec}$ \\
\hline Water Density, $\rho$ & $1.0 \times 10^{3}$ & $\mathrm{~kg} / \mathrm{m}^{3}$ \\
\hline Roughness Parameter, B & 2.5 & \\
\hline Porosity, $\epsilon$ & 0.7 & $\mathrm{~m} / \mathrm{sec}^{3}$ \\
\hline Soil Hyd. Conductivity, $\mathrm{k}_{\text {soil }}$ & $5.8 \times 10^{-5}$ & $\mathrm{~m} / \mathrm{sec}^{2}$ \\
\hline Gravitational Acc., $\mathrm{g}$ & 9.81 & $\mathrm{~m}$ \\
\hline SLOP Basin Height & 7.6 & $\mathrm{~m}$ \\
\hline SLOP Basin Width & 2.6 & $\mathrm{~m}$ \\
\hline SLOP Basin Length & 3.3 & $\mathrm{~m}$ \\
\hline SLOP Basin Perimeter, $\mathrm{p}$ & 5.9 & $\mathrm{~m}$ \\
\hline SLOP Basin Wall Thickness & 0.406 & \\
\hline
\end{tabular}


Table D-2. Volumetric Flow Rate for Flow Through a Porous Medium for Various Floor/Wall Gap Distances

\begin{tabular}{|c|c|c|c|c|c|}
\hline \multicolumn{2}{|c|}{$\begin{array}{l}\text { FloorlWall Gap } \\
\text { Oistance }\end{array}$} & \multicolumn{2}{|c|}{$\begin{array}{l}\text { Flow Through Porous Medium } \\
\text { Whthout Soil Resistance }\end{array}$} & \multicolumn{2}{|c|}{$\begin{array}{l}\text { Flow Through Porous Medium with } \\
.8 \text { Soll resistance }\end{array}$} \\
\hline d & d & 0 & 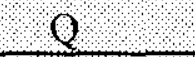 & 9 & 9 \\
\hline$(\mathrm{mm})$ & $(\mathrm{m})$ & $\left(\mathrm{m}^{3} / \mathrm{sec}\right)$ & $(\mathrm{gpm})$ & $\left(\mathrm{n}^{3} / \mathrm{sec}\right)$ & (gpm) \\
\hline 0.1 & 0.0039 & $5.1 \times 10^{-5}$ & 0.8 & $4.8 \times 19^{-5}$ & 0.8 \\
\hline 0.2 & 0.0079 & $1.9 \times 10^{-4}$ & 3.0 & $1.6 \times 10^{-4}$ & 2.5 \\
\hline 0.5 & 0.0197 & $8.3 \times 10^{-4}$ & 13 & $4.5 \times 10^{-4}$ & 7.1 \\
\hline 0.6 & 0.024 & $1.2 \times 10^{-3}$ & 19 & $5.3 \times 10^{-4}$ & 8.3 \\
\hline 1.0 & 0.039 & $2.4 \times 10^{-3}$ & 38 & $6.1 \times 10^{-4}$ & 9.7 \\
\hline 2.0 & 0.079 & $6.8 \times 10^{-3}$ & 108 & $6.5 \times 10^{-4}$ & 10.3 \\
\hline 5.0 & 0.197 & $2.7 \times 10^{-2}$ & 430 & $6.6 \times 10^{-4}$ & 10.4 \\
\hline 10.0 & 0.394 & $7.7 \times 10^{-2}$ & 1217 & $6.6 \times 10^{-4}$ & 10.4 \\
\hline
\end{tabular}

When the soil resistance is included in the pressure drop, the volumetric flow rate is substantially reduced for larger floor/wall gap distances. As noted above, the volumetric flow rate asymptotes to a constant value for floor/wall gap distances larger than $5 \mathrm{~mm}$ (0.197 in).

Reported values of the soil permeability around the Hanford site vary significantly as reported by Kee (1997). A value of $\mathrm{k}_{\mathrm{s}}=14.8 \mathrm{~cm} / \mathrm{hr}(0.00013 \mathrm{ft} / \mathrm{sec})$ was reported as the log-based mean of 16 measured values for the Hanford formation gravels in the 200 Areas (Khaleel and Freeman 1995). However, the soil permeability outside of the immediate wall of the SLOP K Basin is unknown. Even though it is recognized that there will be some resistance to the flow imposed by the soil outside of the wall this contribution is neglected in this uncertainty analysis. Consequently, the uncertainty analysis evaluates the initial flow rate through the floor/wall gap caused by the impact energy of dropping a cask and MCO. The uncertainty analysis does not consider the continuing flow rate that would result from the changing pressure head of the $\mathrm{K}$ Basin water level impeded by soil resistance.

The parameters in Equation D-6 that are represented by uncertainty probability density functions are the porosity $(\epsilon)$ and the roughness parameter $(B)$. All other parameters in Equation D-6 are for water or physical parameters for the K Basin SLOP. The soil saturated hydraulic conductivity is not included because the pressure drop due to soil resistance was not included in the analysis. 
A value of the porosity of 0.7 would represent a fairly dense medium. Values of the porosity of various materials are given in Table D-3. Note that clay has a porosity of 0.45 while sand and gravel has a porosity of 0.2 . For the uncertainty analysis the porosity of the rubbalized concrete in the floor/wall gap was represented by a uniform probability density function ranging from 0.2 to 0.7 . The uniform probability density means that any value of the porosity from 0.2 to 0.7 is equally likely.

The roughness parameter could range from 2 for smooth particles to 4 for rough particles in a porous medium. For the uncertainty analysis the roughness parameter was represented by a uniform probability density function ranging from 2 to 4 . Again this means than any value from 2 to 4 is equally likely.

Table D-3. Ranges of the Value of the Porosity

\begin{tabular}{|l|c|}
\hline System & Porosity \\
\hline Clay & 0.45 \\
\hline Sand & 0.35 \\
\hline Gravel & 0.25 \\
\hline Sand and Gravel & 0.20 \\
\hline Sandstone & 0.15 \\
\hline Quartzite / Granite & 0.01 \\
\hline
\end{tabular}

\section{D.4 Contribution Due to the Water Stop}

As noted in Figure D-1 there is a water stop located midway in the concrete wall that was used as a flow limiter in fabrication of the floor wall joint. The primary function of a water stop is to prevent water from escaping through the concrete construction or contraction joint across which the water stop is installed. The construction documents for the 105-K Building (H-1-21072, HW-4754) indicate that the water stop installed in the K-West Basin SLOP at the joint between the concrete floor slab and the concrete wall is a rubber dumbbell, 2-bulb type. The water stop consists of a basic polymer of either natural rubber or a co-polymer of butadiene and styrene or a blend of both. The water stop was specified to have the following characteristics:

- Tensile strength (minimum) $2500 \mathrm{psi}$

- $\quad$ Elongation at break (minimum) 450 percent. 
The water stop specification stated that "all splices shall be at least equal to the performance and physical characteristics of adjoining unspliced stops (except that the tensile strength may be reduced 50 percent)."

The joint between the concrete floor slab and the wall in the K Basin SLOP was designed as a construction joint and was expected to incur little or no movement. Thus, the water stop would not need to be designed to accommodate any floor/wall movement. The dumbbell 2-bulb type water stop used in this application relies on the bulbs at the ends of the section to provide anchorage in the concrete. When tension is applied to this water stop shape, the web portion of the water stop is reduced in thickness, leaving only the bulbs at the ends in contact with the concrete. Since concrete is not completely impervious, some water may seep past the small area along the web portion of the water stop.

Comparative leak tests have been performed on different shapes of water stops (Kellam 1959). These leak tests indicated that leakage rates with a $1 / 8$-in $(3 \mathrm{~mm})$ joint opening were almost non-existent but that "The dumbbell samples, while being watertight with closed joints, almost without exception leaked with a $1 / 2$-in joint opening." However, they also indicated that when the joint was closed and an additional test run, the joints were effectively watertight.

Another concern would be the condition of the existing K-Basin water stops, which are almost 50 years old. Rubber water stops are longest lived when they are completely out of contact with oxygen and protected from sunlight. When exposed to oxygen or sunlight, rubber water stops harden and crack. The conditions existing inside the K-Basins concrete create a favorable environment for the water stop material, there is no sunlight and there is minimal exposure to oxygen.

The water stops in the K Basin SLOP could be damaged or torn by the motion which would separate the concrete floor from the concrete wall. The most likely damage would be vertical tears in the water stop as the aged material is stretched and placed in tension. The aged material may not have a comparable elongation property as the initial material ( $450 \%$ elongation). Further there could be openings created around the bulbs at each end of the water stop around which water could seep.

The contribution of the water stop was represented as follows. The flow area through the floor/wall gap, which includes the exposed area of the water stop, was represented by;

$$
A=p d(1-F),
$$

where as before $\mathrm{p}$ is the basin perimeter, $\mathrm{d}$ is the floor/wall gap distance. $\mathrm{F}$ is the fraction of the flow area that is not available for water flow due to the presence of a portion of the water stop that is intact. If $F=1$, then $1-F=0$ which means that there is zero flow area that is available for 
water flow. The fraction of flow area not available with the presence of the water stop was represented by a uniform probability density function with values from 0 to 1 . A fraction of flow area not available of $1(1-1=0)$ means that the water stop was completely effective and there is essentially no flow area. A fraction of flow area not available of $0(1-0=1)$ means that the water stop is not effective and the full flow area is available for water flow. The uniform probability density function means that any value of the fraction of area not available for water flow is equally likely. 


\section{Appendix E}

\section{Uncertainty Analysis Results}




\section{E.1 Introduction}

In this appendix the information discussed in each of the other appendices is brought together to form a model which is evaluated using a Monte Carlo convolution process. Several probability density functions which represent specific information relative to the analysis are combined to provide the results that may be expected should a cask and $\mathrm{MCO}$ be dropped into the K Basin SLOP. Each of the probability density functions are representative of conditional probabilities. That is, probabilities that are conditional on a previous event occurring.

The conditional probabilities that are combined in the final model are: (1) the probability of an impact energy given a load drop (Appendix A), (2) the residual floor/wall gap dependence on the impact energy (Appendix B and C), (3) the probability of the residual floor/wall gap given an impact energy (Appendix C), and (4) the volumetric flow rate through the floor/wall gap given a residual floor/wall gap (based on Appendix D). The convolution model combines each of these probability density functions which in turn are based on the uncertainty associated with each of the variables involved in each calculation. In this analysis it was expeditious to partition the solution into the various sections that were discussed in each appendix. In reality, a composite model could have been developed which would have combined all of the analysis together into one model. However, for this analysis it was more instructive to examine each portion of the analysis to gain additional insight into the results.

\section{E.2 Results for the Initial Volumetric Flow Rate}

Each of the conditional probabilities identified above were sampled 10,000 times to provide a floor/wall gap distance. Based on the floor/wall gap distance, the initial flow rate was calculated using the equations and appropriate variable uncertainties presented in Appendix D. The superficial velocity was determined and used to calculate an initial volumetric flow rate through a porous medium. The volumetric flow rate was calculated for each of the 10,000 iterations in $\mathrm{m}^{3} / \mathrm{sec}$, but was converted to gallons per minute for presentation of the results. The probability density function for the distribution for the volumetric flow rate is provided in Figure E-1. The corresponding cumulative distribution function is provided in Figure E-2. The probability density function that represents the initial volumetric flow rate, for larger flows, is exponentially decreasing. However, the best fit to all of the data is a Lognormal probability density function with log-mean of 3.79 and a log-standard deviation of 1.57 , which relates to a mean of $51.2 \mathrm{gpm}$ and a standard deviation of $29.8 \mathrm{gpm}$. A summary of the results presented in Figures E-1 and E2 is given in Table E-1. 


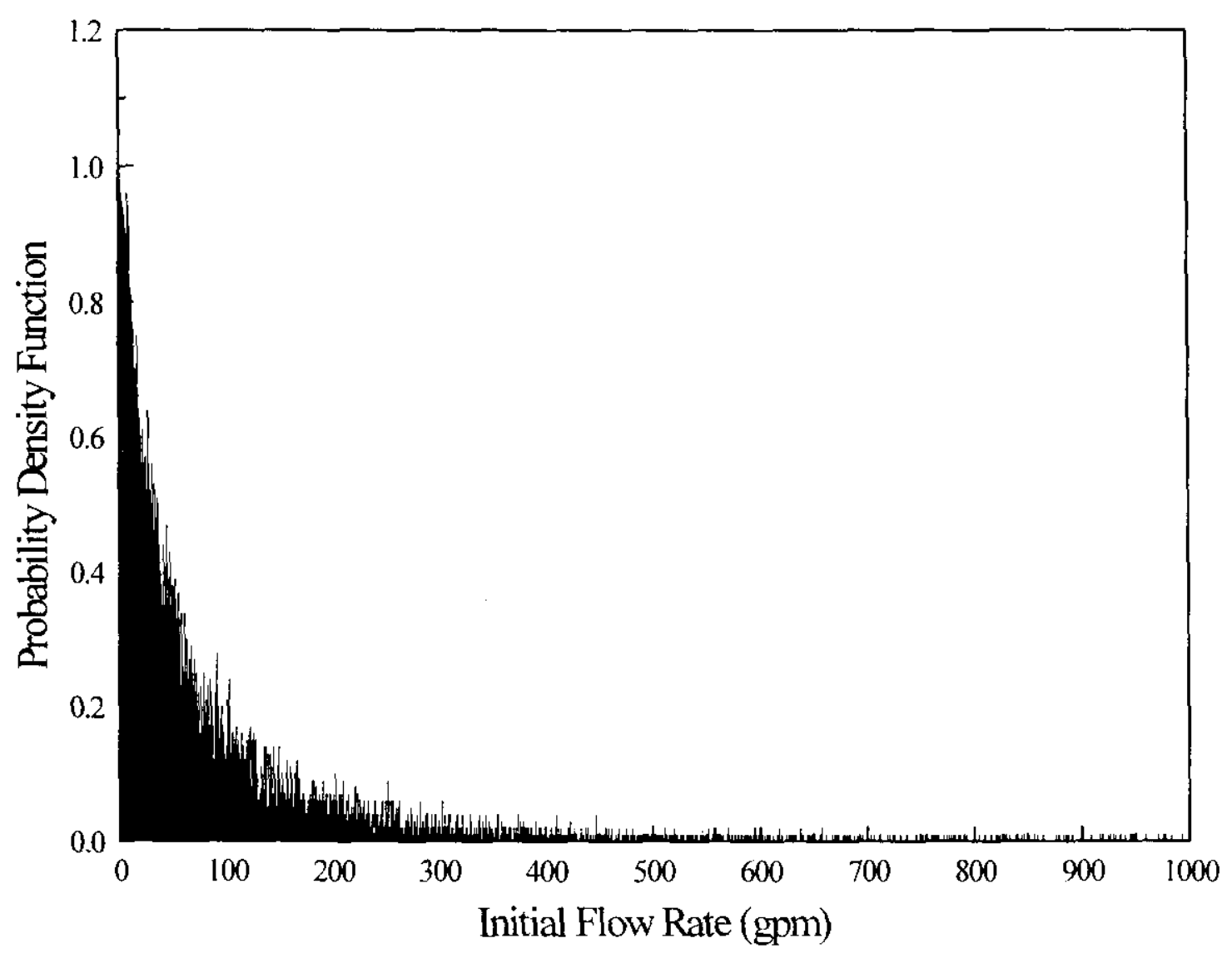

Figure E-1. Probability Density Function for the Initial Volumetric Flow Rate. 


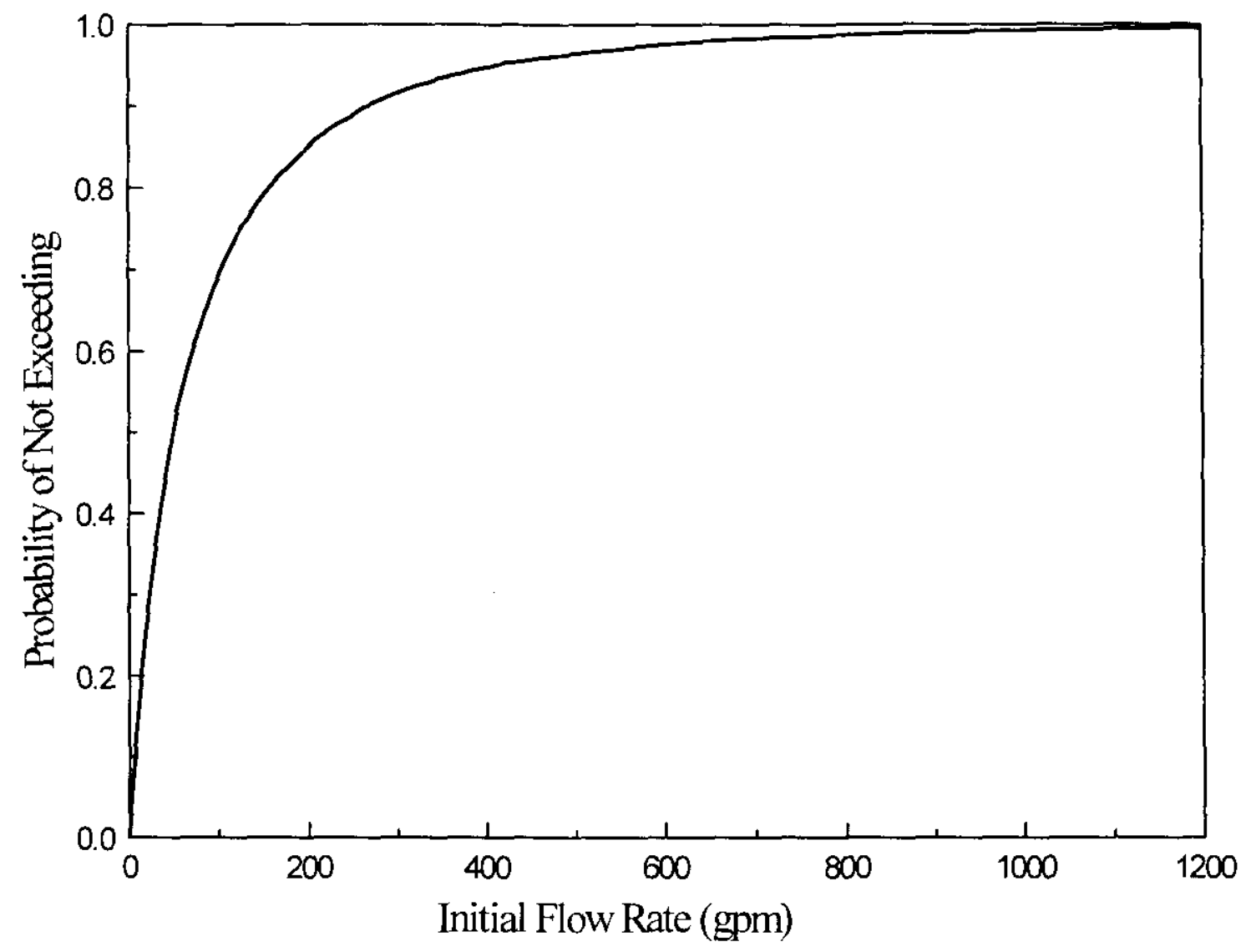

Figure E-2. Cumulative Distribution Function for the Initial Volumetric Flow Rate. 
Table E-1. Probability of Not Exceeding a Specified Initial Volumetric Flow Rate

\begin{tabular}{|l|c|c|}
\hline 14 & Probability & Initial Volumetric Flow Rate Is Less \\
\hline Mean & & Than (gpm) \\
\hline 50 th Percentile & 0.50 & 51 \\
\hline 90th Percentile & 0.90 & 268 \\
\hline 95th Percentile & 0.95 & 411 \\
\hline 99th Percentile & 0.99 & 916 \\
\hline
\end{tabular}

From Table E-1, for example, there is a probability of 0.95 that the volumetric flow rate will be less than $411 \mathrm{gpm}$. The associated percentile figures in Table E-1 indicate, for example, that 95 percent of the time the volumetric flow rate was less than $411 \mathrm{gpm}$. Stated another way, given the conditional probability that a cask and $\mathrm{MCO}$ have been dropped in the K-West Basin SLOP, there is a $95 \%$ confidence that the resulting initial volumetric flow rate will be less than $411 \mathrm{gpm}$. In the analysis there were a few volumetric flow rates that were as large as $1,000 \mathrm{gpm}$ to 2,000 $\mathrm{gpm}$, but these flow rates occurred less than $1 \%$ of the time. Hence, as noted from Table E-1, $99 \%$ of the time the volumetric flow rate is less than $916 \mathrm{gpm}$, or there is a $99 \%$ confidence that the initial volumetric flow rate will be less than $916 \mathrm{gpm}$.

A sensitivity analysis was completed to identify the variables that contribute the most to the uncertainty in the initial volumetric flow rate. The results are as expected. The uncertainty in the initial volumetric flow rate is predominantly due to the height at which a cask and MCO may be dropped into the K-Basin SLOP. The uncertainty in the cask drop height is reflected in the uncertainty in the floor/wall gap thickness. Other variables that provide significant contributions to the uncertainty in the initial volumetric flow rate are the effectiveness of the waterstop and the porosity of the concrete rubble in the floor/wall gap. Therefore, as expected, the most effective way to prevent any water flow from the $\mathrm{K}$-Basins is to prevent a cask and $\mathrm{MCO}$ from being dropped. 


\section{Appendix F \\ Validation of Analysis Models}




\section{F.1 Introduction}

This appendix provides the documentation for the functional reviews and specific hand calculations to demonstrate that the model analysis is correct.

\section{F.2 Functional Reviews}

During the functional reviews, the client decided that an appendix that was provided with the original draft report should be removed from the document. This appendix, original Appendix A, SLOP Transfer Bay Crane Fault Tree Analysis, determined the distribution for the probability of dropping a cask and MCO into the K-West Basin South Loadout Pit (SLOP) while using the $\mathrm{K}$-West Basin transfer bay crane. Therefore, the focus of the present report was changed to consider the conditional probability that a cask and $\mathrm{MCO}$ had been dropped and then determine the subsequent results in terms of the initial volumetric flow rate from the $\mathrm{K}$ Basin.

Comments resulting from the functional reviews are documented on Review Comment Records (RCRs). The report was modified as appropriate to incorporate these comments. Attachment F1 provides the approved and signed RCRs.

\section{F.3 Validation of Models}

The calculations used in Appendix A, C, D, and E were checked for appropriateness and accuracy. In most cases point value solutions were checked by appropriate hand calculations and/or spreadsheet calculations. Attachment F-2 provides the checked calculations and/or calculation notes. 


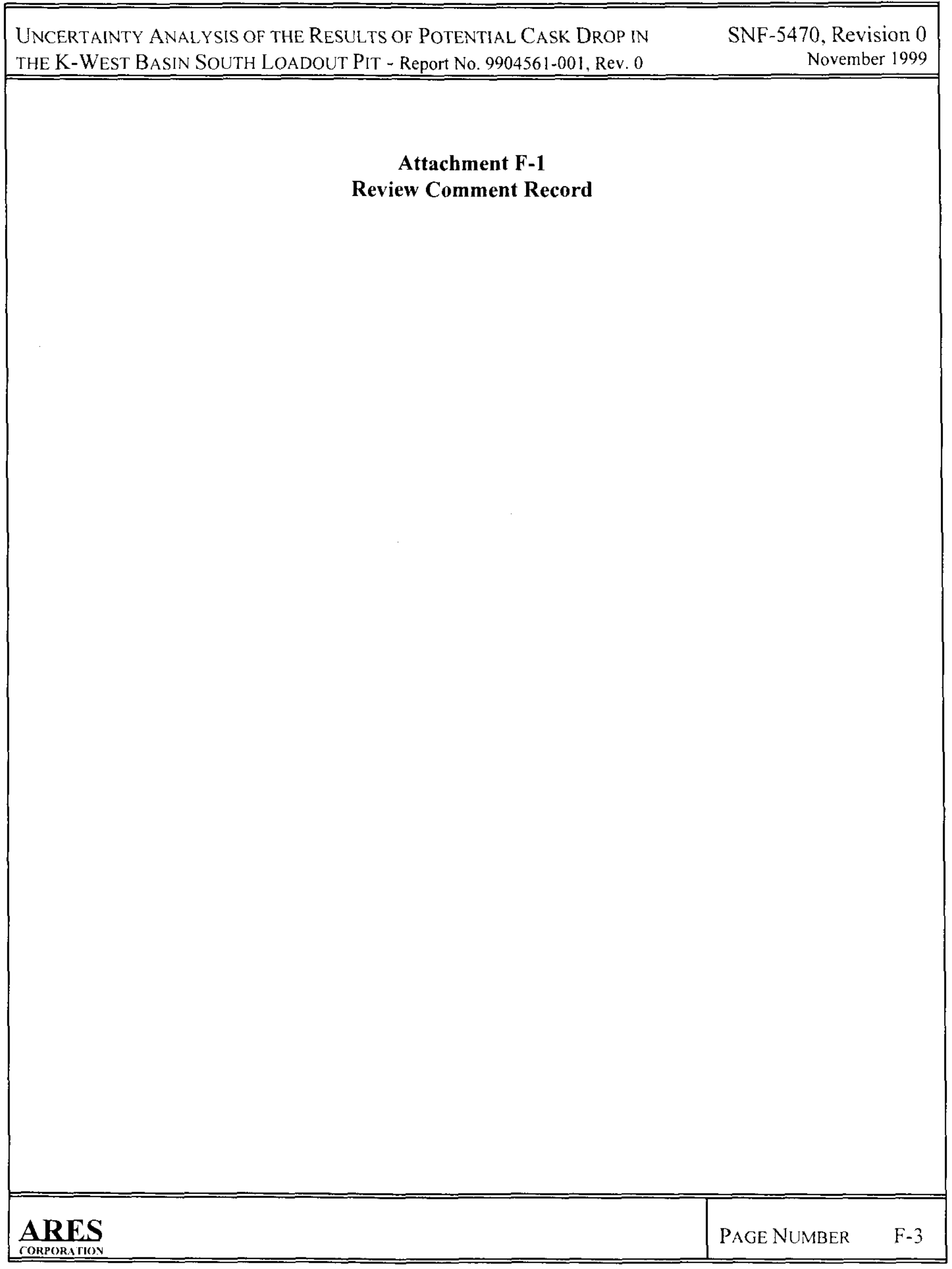


SNF-5470, Revision 0

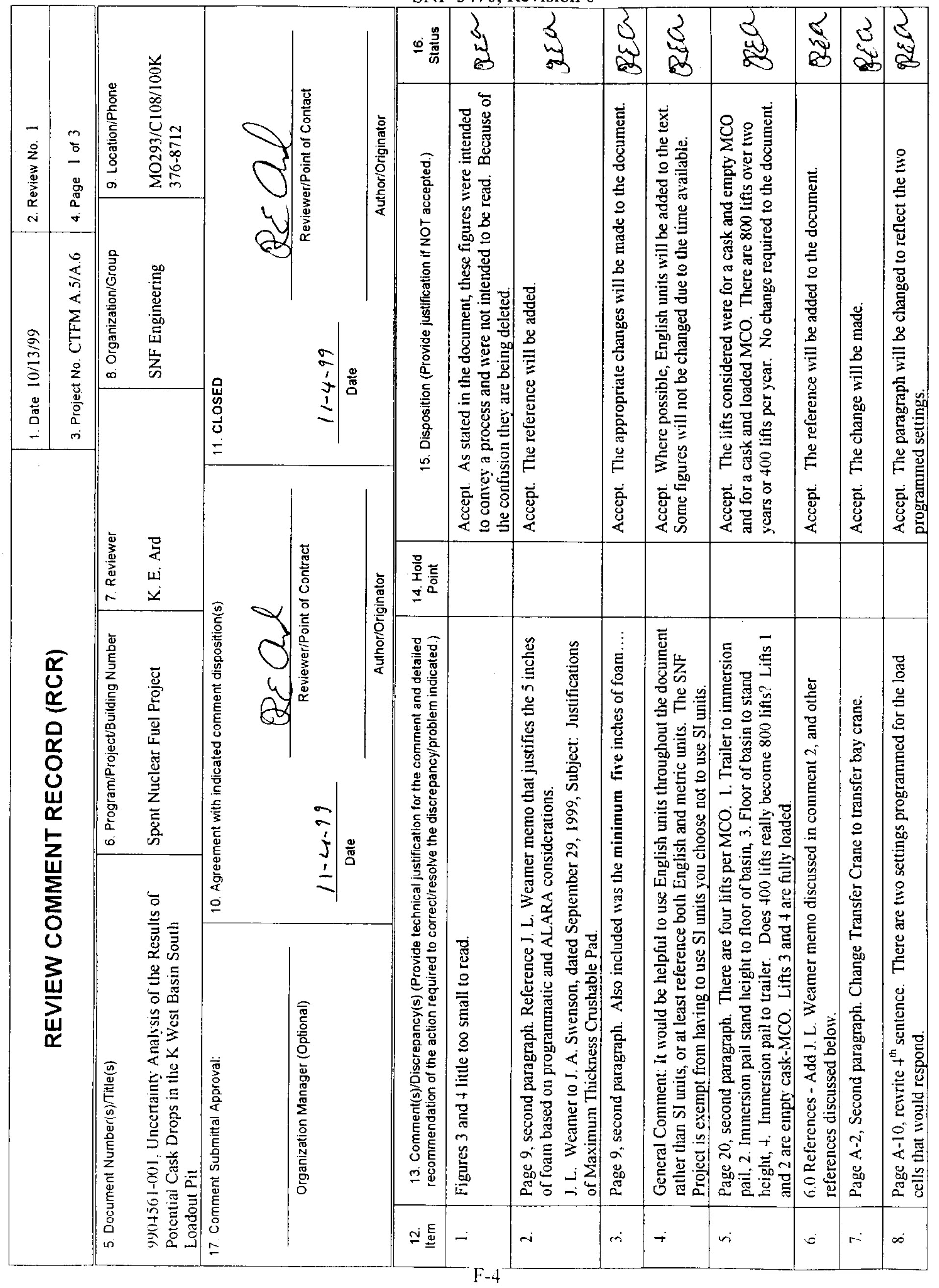


SNF-5470, Revision 0

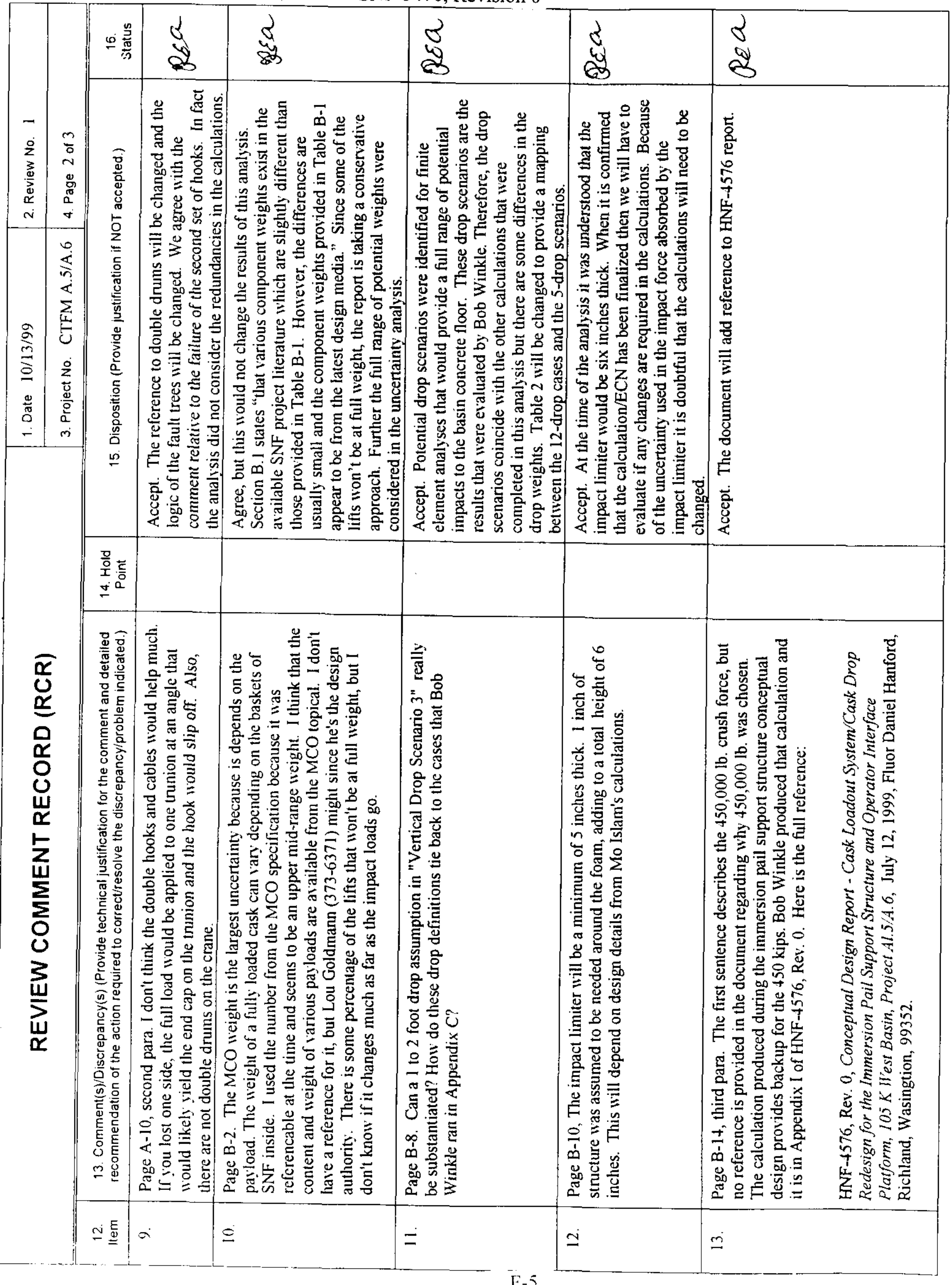




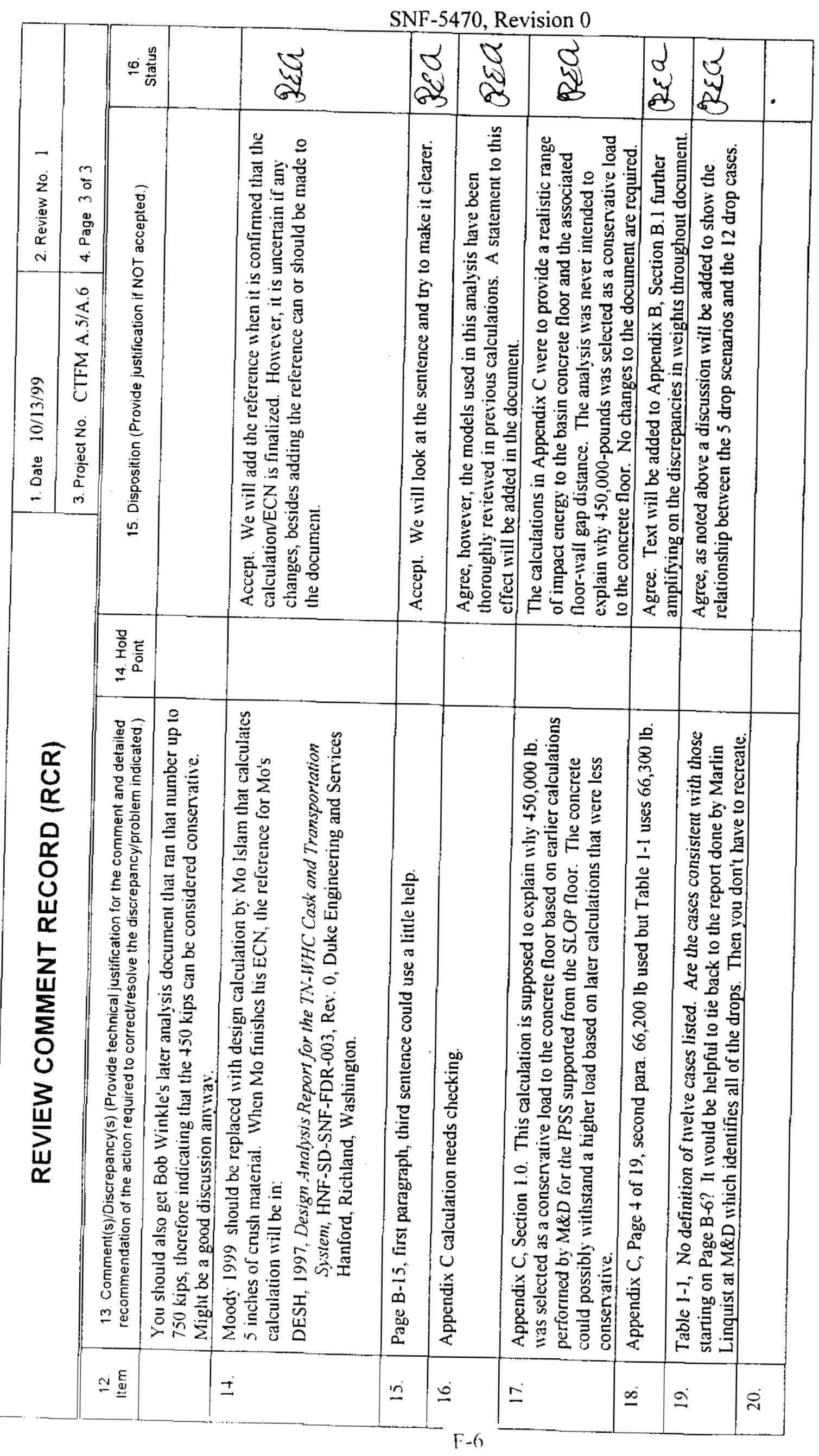




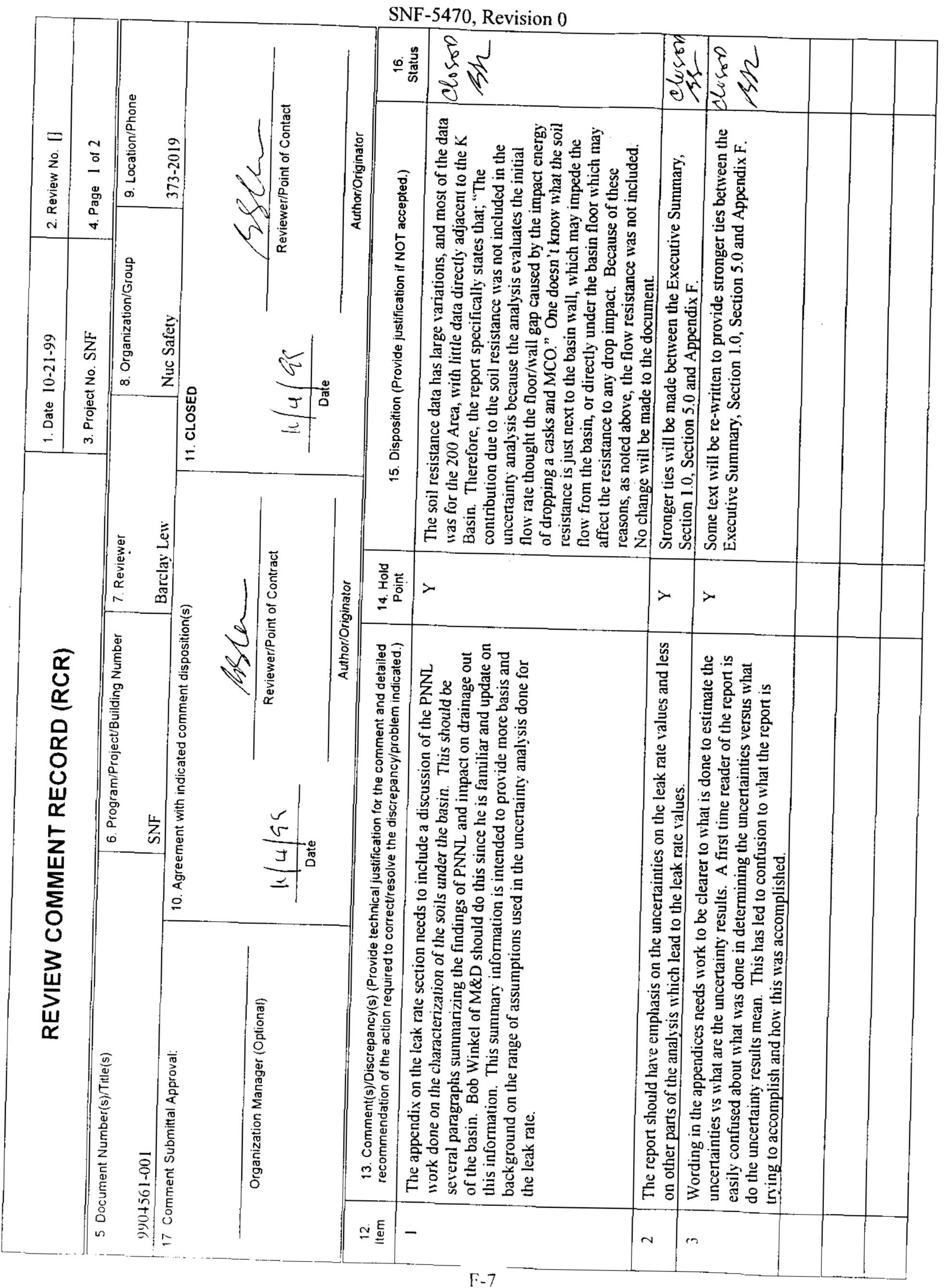


SNF-5470, Revision 0

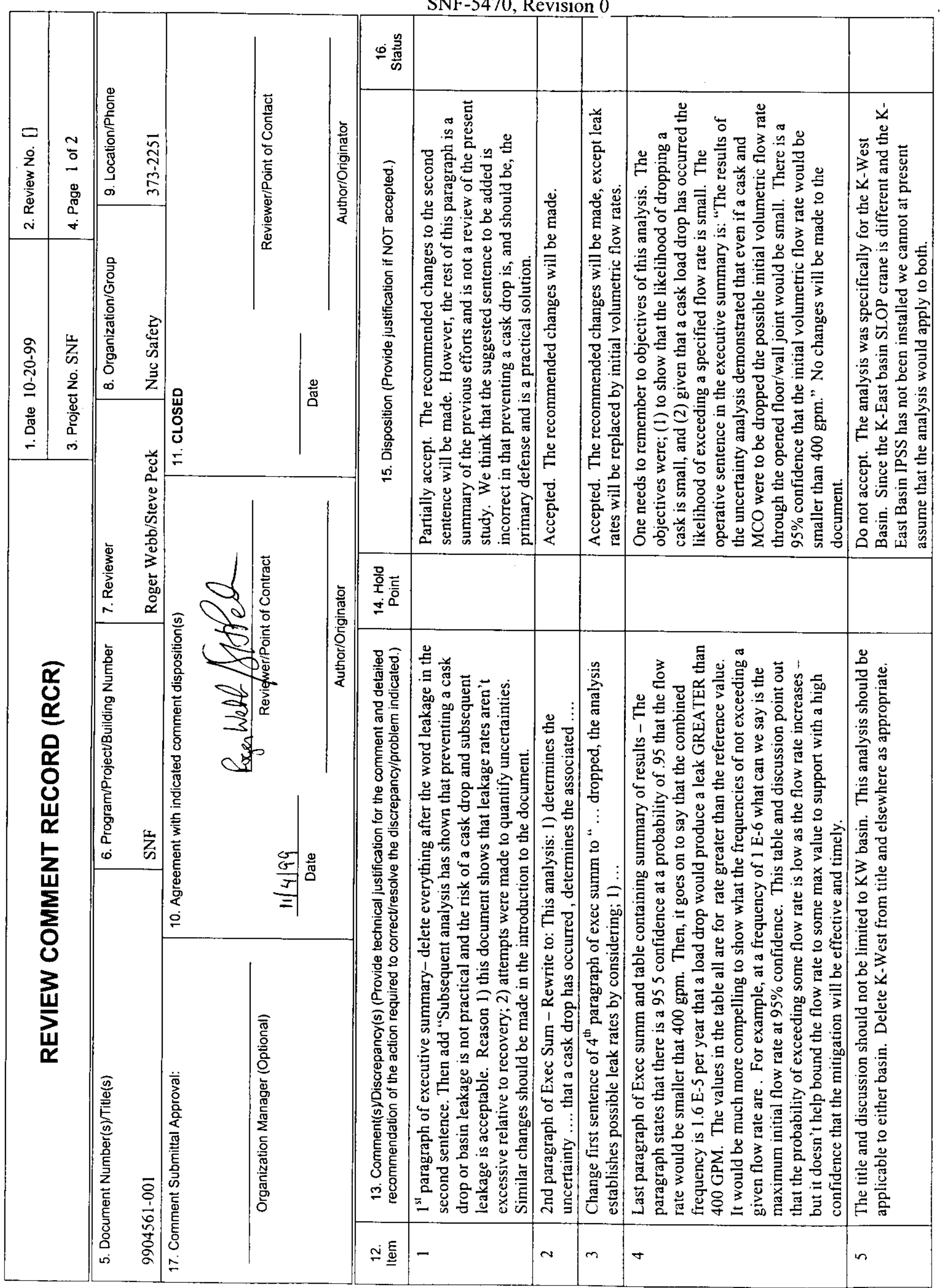




\section{Attachment F-2 \\ Checked Calculations and Calculation Notes}

\begin{tabular}{|l|ll|}
\hline $\mathbf{A R E S}$ & PAGE NUMBER & F-9 \\
\hline
\end{tabular}




\section{Formulas Used For Model Verification}

The following formulas were used in verifying the model calculations.

The velocily of the Cask and MCO falling in air just prior to impact with the buttom of the

Immersion Pail is given by;

$$
V_{1}-\sqrt{2 \mathrm{BH}_{1}} \text {. }
$$

The velocity of the Cask and MCO immediately after impact with the botton of the Immersion Pail is given by:

$$
v_{2}=\sqrt{v_{1}^{2}-\frac{2 U}{m}}
$$

where $U$ is the energy lost due to shearing the Immersion Pail pins.

The velocity of the Cask, MCO and Immersion Pail just prior to impacting the bottom of the Immersion Pail Support Structure is calculated by; $V_{3}$ calculated using Equation $\Lambda-14$ of Appendix $A$.

The kinetic energy of the Cask, MCO and Immersion Pail just prior to impacting the bottom of the Immersion Pail Support Structure is given byr;

$$
K F_{1}=\frac{1}{2} \mathrm{~m} \mathrm{~V}_{3}^{2}
$$

The remaining kinetic energy after the eneryy lost by crushing the impact lin iter and shearing the legs of the Immersion Pail Support Structure is calculated froro;

$$
K E_{1}=K E_{1}-U_{\text {impace linirer }}-U_{\text {bottom plate }} \text {. }
$$

This is the impact energy to the K. Basin SL.OP concrete floor.

The following equations are used to calculate the initial volumetric flow thro tgh the $\mathrm{K}$ Basin SLOP loor / wall gap which results from the impact energy of dropping a ca $3 \mathrm{k}, \mathrm{MCO}$ and Immersion Pail.

Referring to Equation D-6 in Appendix 6, the superficial velocity through the floor/wall gap is given by;

$$
u=\frac{-b+\sqrt{b^{2}-4 a c}}{2 a}
$$


where the constants $a, b$. and $c$ are calculated using Equation D-8 in Appentix D.

With the superficial velocity through the foor/wall gap and the floor/wall g, a height, the initial volumetric flow through the floor/wall gap is given by;

$$
Q=A U-d p U F
$$




\begin{tabular}{|c|c|c|}
\hline \multicolumn{3}{|c|}{ Table F-1. Parameters For Model Hand Calculations } \\
\hline Drag Coefficient & 1.2 & \\
\hline Density of Water & 1000 & $\mathrm{~kg} / \mathrm{m}$ \\
\hline Gravitational Const & $9.81 \mathrm{r}$ & $\mathrm{m} / \mathrm{sec} 2$ \\
\hline Frontal Area of IP & $1.2464 \mathrm{r}_{\mathrm{r}}$ & $\mathrm{m} 2$ \\
\hline Volume of Cask \& IP & $4.9128 \mathrm{r}$ & $\mathrm{m} 3$ \\
\hline Constant k & 747.85 & $\mathrm{~kg} / \mathrm{m}$ \\
\hline Cask, MCO; Mass 1 & 58885 & $1 \mathrm{~b}$ \\
\hline Cask, MCO, IP; Mass 2 & 66191 & lb \\
\hline Drop Height $(\mathrm{m})$ & 9.217 & $\mathrm{~m}$ \\
\hline Height 1 & 4.46 & $\mathrm{~m}$ \\
\hline Height 2 & 4.76 & $\mathrm{~m}$ \\
\hline Mass 1 & 26710 & $\mathrm{~kg}$ \\
\hline Mass 2 & 30024 & $\mathrm{~kg}$ \\
\hline Velocity at lmpact to IP & 9.350 & $\mathrm{~m} / \mathrm{sec}$ \\
\hline Energy absorbed to yield pins & 36000 & nt-m \\
\hline Velocity after Impact with IP & 9.20 & $\mathrm{~m} / \mathrm{sec}$ \\
\hline Velocity at Impact to Bottom & 11.679 & $\mathrm{~m} / \mathrm{sec}$ \\
\hline $\mathrm{KE}$ & 2047503 & nt-m \\
\hline Foam & 270000 & nt-m \\
\hline IPSS & 20000 & nt-m \\
\hline Remain & 1757503 & nt-m \\
\hline Viscosity of Water & $1.00 \mathrm{E}-03$ & $\mathrm{~kg} / \mathrm{m}-\mathrm{sec}$ \\
\hline Roughness Parameter & 2.5 & \\
\hline Porosity & 0.7 & $\mathrm{dl}$ \\
\hline SLOP Basin Height & 7.600 & $m$ \\
\hline SLOP Basin Wall Thickness & 0.4064 & \\
\hline SLOP Basin Perimeter & 5.900 & $\mathrm{~m}$ \\
\hline Residual Gap & 1.0000 & $\mathrm{~mm}$ \\
\hline Residual Gap & $1.00 \mathrm{E}-03$ & $\mathrm{~m}$ \\
\hline$a=$ & $1.09 \mathrm{E}+06$ & \\
\hline $\mathrm{b}=$ & $1.18 \mathrm{E}+04$ & \\
\hline$c=$ & -183455 & \\
\hline Superficial Velocity & $4.04 \mathrm{E}-01$ & $\mathrm{~m} / \mathrm{sec}$ \\
\hline Water Stop Effectiveness & 1 & 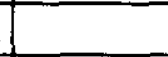 \\
\hline Volumetric Flow Rate & $2.39 \mathrm{E}-03$ & $\mathrm{~m} 3 / \mathrm{sec}$ \\
\hline Volumetric Flow Rate & $3.79 \mathrm{E}+01$ & gpm \\
\hline
\end{tabular}


SNF-5470, Revision 0

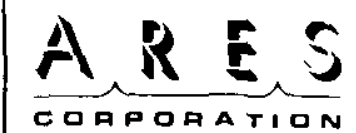

CALCULATION

SHEET

Project No.: 9904500.54 Call. No.: Dol Revision No.: $\varnothing$

SHEET NO: 1

PREPAREDBY: $c M$ consELmAn Date: $11 / 1 / 99$ REVIEWEDBY:

$N / A$

DATE:

HAN Q CALCULATION VALIDATING MODEL CALCULATIONS

$$
\begin{aligned}
& V_{1}=\sqrt{2 g H_{1}}=\sqrt{2(9.81) 4.46}=9.35 \mathrm{~m} / \mathrm{skc} \\
& V_{1}=\sqrt{V_{1}-\frac{2 U}{m}}=\sqrt{9.35^{2}-\frac{2(36,000)}{26,70}}=9.20 \mathrm{~m} / \mathrm{sEC} \\
& V_{3}=\sqrt{V_{0}^{2} e^{\frac{-1 k}{m}(h-z)}-\frac{\rho g V_{0}}{k}\left(1-e^{\frac{-2 k}{m}(h-z)}\right)+\frac{m g}{k}\left(1-e^{-\frac{2 k}{m}(h-z)}\right)}
\end{aligned}
$$

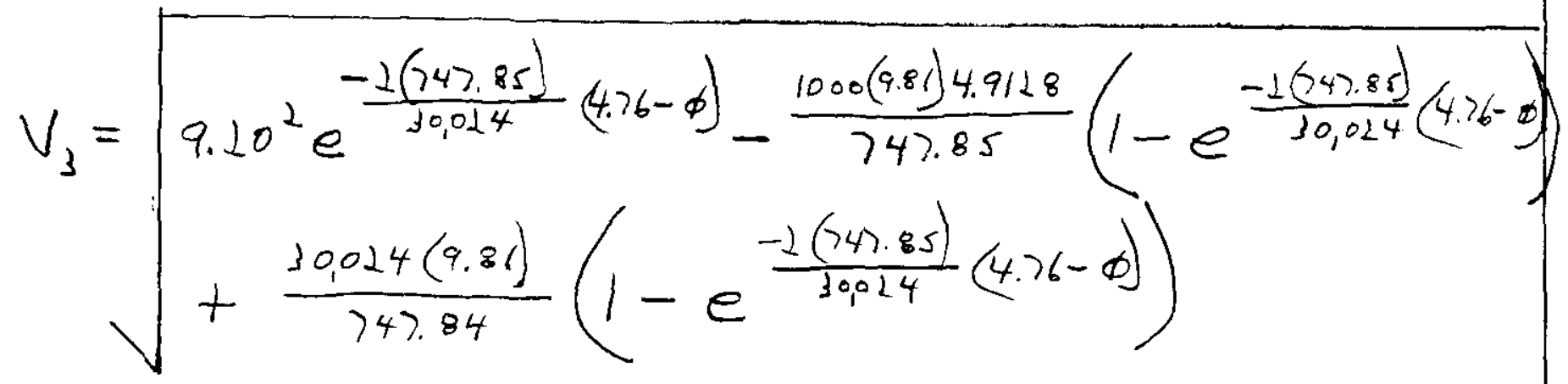

$$
\begin{aligned}
& V_{3}=11.68 \mathrm{~m} / \mathrm{sec} \\
& K E_{1}=1 / 2 m V_{3}^{2}=1 / 2(30,024) \quad 11.68^{2}=2,047,501 N T-M
\end{aligned}
$$

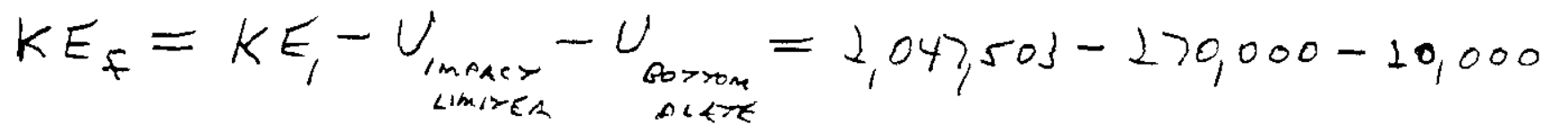

$$
\begin{aligned}
& K E_{f}=1,75 \geqslant, 50,3 N T-M
\end{aligned}
$$


SNF-5470, Revision 0

ARES

CALCULATION

Project No:: 9904500.54 Call. No.: oO l Revision No.:

SHEET

Prepared Br: $c . M$ con se MAN DATE: $1 / / 1 / 99$ ReVIEWEd BY: N/A

SHEet No:: 2

DATE:

$$
\begin{aligned}
& U=\frac{-b+\sqrt{b^{2}-4 a c}}{2 a}=\frac{-1.18 \times 10^{4}+\sqrt{1.18 \times 10^{4}-4\left(1.09 \times 10^{6}\right)-183.455}}{1\left(1.09 \times 10^{6}\right)} \\
& U=0.404 \mathrm{~m} / \mathrm{sEc} \\
& Q=A U=d P U F=\left(\frac{1.0}{1000}\right) 5.9(0.404) 1.0 \\
& Q=2.39 \times 10^{-1} \mathrm{~m}^{3} / \mathrm{sEC}=37.9 \mathrm{GPm}
\end{aligned}
$$

$F-14$ 